\title{
Cytoplasmic labile iron accumulates in aging stem cells perturbing a key rheostat for identity control
}

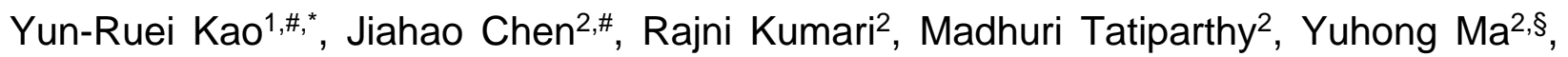
Maria M. Aivalioti2,\$, Aliona Zintiridou², Victor Thiruthuvanathan², Julie A. Reisz ${ }^{3}$, Stephanie Stranski ${ }^{4}$, Simone Sidoli ${ }^{4}$, Ulrich Steidl ${ }^{1,2,5,6}$, Angelo D'Alessandro ${ }^{3}$, Britta Will ${ }^{1,2,5,6,7^{*}}$

${ }^{1}$ Department of Medicine, Albert Einstein College of Medicine, New York, NY, USA; ${ }^{2}$ Department of Cell Biology, Albert Einstein College of Medicine, New York, NY, USA; ${ }^{3}$ Department of Biochemistry and Molecular Genetics, University of Colorado Denver Anschutz Medical Campus, Denver, CO, USA; ${ }^{4}$ Department of Biochemistry, Albert Einstein College of Medicine, New York, NY, USA; ${ }^{5}$ Ruth L. and David S. Gottesman Institute for Stem Cell Research and Regenerative Medicine, Albert Einstein College of Medicine, New York, NY, USA; ${ }^{6}$ Blood Cancer Institute, Albert Einstein Cancer Center, Albert Einstein College of Medicine, New York, NY, USA; ${ }^{7}$ Institute for Aging Studies, Albert Einstein College of Medicine, New York, NY, USA.

$\#, \S$ These authors contributed equally.

Keywords: Adult stem cells; ageing; iron homeostasis; lipid metabolism; histone acetylation; gene regulation.

${ }^{*}$ Corresponding authors:

Britta Will, PhD

Albert Einstein College of Medicine

1300 Morris Park Avenue

New York, NY 10461, USA

Phone: +1 7184303786

Email: britta.will@einsteinmed.org
Yun-Ruei Kao, PhD

Albert Einstein College of Medicine 1300 Morris Park Avenue

New York, NY 10461, USA

Phone: +1 7184306431

Email: yun-ruei.kao@einsteinmed.org 
1 Bone marrow resident and rarely dividing haematopoietic stem cells (HSC) harbour

2 an extensive self-renewal capacity to sustain life-long blood formation; ${ }^{1-5}$ albeit

3 their function declines during ageing. ${ }^{6,7}$ Various molecular mechanisms confer

4 stem cell identity, ensure long-term maintenance and are known to be deregulated

5 in aged stem cells. ${ }^{8,9}$ How these programs are coordinated, particularly during cell

6 division, and what triggers their ageing-associated dysfunction has been unknown.

7 Here, we demonstrate that HSC, containing the lowest amount of cytoplasmic

8 chelatable iron (labile iron pool) ${ }^{10}$ among hematopoietic cells, activate a limited iron

9 response during mitosis. Engagement of this iron homeostasis pathway elicits mobilization and $\beta$-oxidation of arachidonic acid and enhances stem cell-defining

11 transcriptional programs governed by histone acetyl transferase Tip60/KAT5. We

12 further find an age-associated expansion of the labile iron pool, along with loss of

13 Tip60/KAT5-dependent gene regulation to contribute to the functional decline of ageing HSC, which can be mitigated by iron chelation. Together, our work reveals cytoplasmic redox active iron as a novel rheostat in adult stem cells; it demonstrates a role for the intracellular labile iron pool in coordinating a cascade of molecular events which reinforces HSC identity during cell division and to drive stem cell ageing when perturbed. As loss of iron homeostasis is commonly observed in the elderly, we anticipate these findings to trigger further studies into understanding and therapeutic mitigation of labile iron pool-dependent stem cell dysfunction in a wide range of degenerative and malignant pathologies. 
24 the active cell cycle and undergo cell division to contribute to mature blood cell production or replenish the stem cell pool ${ }^{13,14}$. Accumulation of genetic and epigenetic generegulatory alterations occur in HSC over time and multiple replications ${ }^{15,16}$. They, along

27 with various extracellular changes ${ }^{17-19}$, contribute to the ageing-related decline of 28 functional stem cells ${ }^{20}$ which are the cellular source of several degenerative ${ }^{21}$ and 29 malignant pathologies 22.

Past studies have provided crucial insights into the molecular and cellular mechanisms governing long-term maintenance of adult stem cells; these include, ability to undergo and modulate cell division modes (asymmetric vs. symmetric) ${ }^{23-25}$, reliance on glycolytic pathways ${ }^{26}$ and autophagy ${ }^{9,27}$ for curtailing reactive oxygen species (ROS)

34 generation while producing sufficient energy and cellular building blocks ${ }^{28,29}$, and low protein translation rates $^{30-33}$. Each cell division demands wide-ranging metabolic and gene-regulatory adaptations to meet the changing energetic and structural demands of

37 mitosis $^{34-38}$. As these adaptations are largely incompatible with their sustained long-term 38 function, stem cells must counteract mitosis-related molecular perturbations - the molecular processes at play have remained elusive thus far.

41 required in numerous electron transfer reactions governing fundamental cellular 42 processes $^{39}$, cells tightly regulate the size of the intracellular labile iron pool (LIP) ${ }^{10}$ to limit 43 adverse ROS generation ${ }^{40,41}$. Perturbations in the ability to limit intracellular iron is 44 detrimental for cells ${ }^{42}$ and known to compromise HSC maintenance and function via 45 altered redox signalling and increased macromolecule oxidation and damage ${ }^{43}$. We have 46 previously uncovered that iron chelator exposure increases the number of functional HSC 
47 ex vivo and in vivo ${ }^{44}$. The HSC stimulatory effects of iron chelator (IC) treatment and the well characterized central roles of redox active intracellular iron in sustaining basic cell function prompted us to examine a potential regulatory role of the LIP in controlling somatic stem cell function.

HSC contain the most limited LIP among hematopoietic cells

52 The size of readily accessible functional intracellular iron pools is determined by the cellular state and function of cells ${ }^{45,46}$. We first quantified the amount of intracellular

54 chelatable, redox active ferrous iron $\left(\mathrm{Fe}^{2+}\right)$ species across various haematopoietic cell types which revealed differences in their LIP. Compared to highly proliferative Lineage ${ }^{-}$ $\left(\mathrm{Lin}^{-}\right)$and myeloid progenitor cells ( $\left.\mathrm{Lin}^{-} \mathrm{CKit}^{+} \mathrm{Sca}-1^{-}, \mathrm{LK}\right)$, most immature hematopoietic

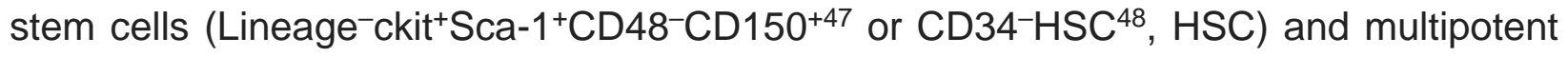
progenitor cells (Lineage ${ }^{-}$ckit $^{+}$Sca- $1^{+}$CD48-CD150 ${ }^{-47}$, MPP) harbour well detectable, but significantly lower amounts of free $\mathrm{Fe}^{2+}$ (Extended Data Fig. 1a), consistent with their mostly quiescent state and lower energetic requirements ${ }^{26,49-52}$. and at comparable levels in mouse HSC and maturing progenitor cell populations (Extended Data Fig. 1b-d); notable exceptions are iron storage genes Ferritin Heavy Chain 1 (Fth1) and Ferritin Light Chain (Ft/1) which are found expressed at higher levels in HSC than in more mature progenitor cells, while iron sensors Aconitase 1 / Iron responsive protein 1 (Aco1/Irp1) and Iron Responsive Element Binding Protein 2 (Ireb2)

67 and the Transferrin Receptor (Tfrc), relevant for cellular iron uptake, showed slightly lower 68 abundance in HSC compared to their mature progeny (Extended Data Fig. 1b-d). 
presentation further showed that HSC harboured an approximately $70 \%$ lower total

71 protein content than erythro-myeloid lineage-committed progenitor cells, in line with

72 previous observations 25,53 ; notably, our analyses also revealed that only a minor fraction

73 of the receptor is presented on the surface of all cell types tested (Extended Data Fig.

74 1e,f). These data support a lower labile iron requirement and a bias towards iron storage

75 in HSC under steady state conditions.

\section{Limited iron response activation in mitotic stem cells}

77 A decrease in intracellular labile iron content is sensed and corrected by the iron responsive protein (IRP) / iron responsive element (IRE) system which post-

79 transcriptionally controls the expression and translation of several mRNA transcripts encoding proteins of iron-, oxygen- and energy metabolism, including the stabilization of Tfrc transcripts (limited iron response) ${ }^{54}$. Motif prediction in mouse and human HSC gene expression signatures showed a significant enrichment of IRE bearing transcripts to a similar extent as found when using gene expression signatures of erythroid cells whose

84 function critically relies on $\operatorname{iron}^{55-57}$ (Fig. 1a,b, Extended Data Fig. 1g-j). We noted that almost a quarter of differentially expressed transcripts in proliferating HSC (vs. quiescent stem cells) contained IRE motifs (Fig. 1c, Extended Data Fig. 1k), suggesting an activation of the IRP/IRE-mediated limited iron response in proliferating HSC. To test this prediction, we quantified cytoplasmic and nuclear Tfrc transcript levels, as well as

89 transcription dynamics at single cell and single-molecule resolution. We utilized singlemolecule RNA fluorescence in situ hybridization (smRNA FISH) technology, which we

91 have reported and validated in the hematopoietic system previously ${ }^{58}$, in a mouse HSC 92 cell line, HPC-7 (Fig. 1d, Extended Data Fig. 2a-g). Compared to non-mitotic cells, 
93 mitotic HSC showed a significant increase in cytoplasmic mRNA levels (Fig. 1e,

94 Extended Data Fig. 2h) while displaying no signs of increased transcriptional activity at

95 the Tfrc locus as evidenced by unchanged transcriptional burst size and frequency

96 (Extended Data Fig. 2i,j). Experimental decrease of intracellular iron through exposure

97 to an IC (deferoxamine (DFO)) showed similar results (Extended Data Fig. 2k-n). These

98 observations revealed that the increased abundance of Tfrc is mediated through enhanced mRNA stabilization ${ }^{54}$ and strongly suggested that iron limitation triggers a compensatory iron homeostasis reaction in dividing HSC.

102 for which we employed short term ex vivo IC treatment of primary HSC and HPC-7 cells.

103 Compared to vehicle treated controls, IC exposure to a rapid decrease in Fth1 and Ftl

104 protein abundance (Fig. 1f, Extended Data Fig. 3a) within one hour, activation of 105 intracellular iron storage mobilization by Ncoa4-dependent ferritinophagy ${ }^{59}$ (Fig. 1g,

106 Extended Data Fig. 3b) and was associated with elevated and readily restored 107 intracellular $\mathrm{Fe}^{2+}$ levels within only four hours of IC exposure (Extended Data Fig. 3c). 108 We also found alterations in mRNA expression consistent with reduced intracellular iron 109 content after IC exposure of primary HSC for 16 hrs (Extended Data Fig. 3d-f, Extended 110 Data Table 2). Notably, stem cells did not show a detectable increase in the uptake of 111 extracellular iron before 8 hrs (Fig. 1h) or elevated CD71 cell surface presentation before 1126 hrs (Extended Data Fig. 3g) after IC treatment. Yet, we also noted that intracellular 113 iron stores seem smaller in hematopoietic stem and multipotent stem cells as compared 114 to their more mature progeny (Extended Data Fig. 3h). This set of data strongly suggests 115 that upon activation of the limited iron response HSC, first increase intracellular iron 
116 mobilization before upregulating CD71 which has previously been associated with

117 enhanced differentiation commitment ${ }^{25,53}$.

118 Activation of the limited iron response increases HSC function

119 To test whether alterations in LIP size and activation of the limited iron response has

120 functional consequences in HSC, we exposed highly purified stem cells to IC ex vivo for

12148 hrs and subsequently preformed single cell transcriptomic analysis. Compared to

122 vehicle treated control HSC cultures, IC treated cells showed a larger proportion of cells

123 retaining HSC-typic gene expression signatures (Fig. 1i, Extended Data Fig. 4a), with

124 increased scores of gene signatures for HSC proliferation, and Stem Cell identity, as well

125 as concomitantly decreased scores for HSC differentiation and quiescence (Fig. 1j,k,

126 Extended Data Fig. 4b-d, Extended Data Table 1). We next subjected mice to a 14-day

127 regimen of daily treatment with iron chelator DFO ${ }^{60,61}$. Compared to mock-treated

128 controls, this treatment regimen led to reduced body iron without triggering overt iron

129 deficiency pathology (Extended Data Fig. 4e-g). Hematopoietic stem and multipotent

130 progenitor cell (HSPC) activity, assessed by long-term culture-initiating cell (LTC-IC)

131 quantification, was found increased in IC-treated mice compared to mock control animals

132 (Extended Data Fig. 4h), confirming our previous observations with human HSPC and 133 additional iron chleators ${ }^{44}$. These findings indicated that IC exposed cells retained more

134 effectively their stem cell-typic gene expression programs and function compared to 135 vehicle controls.

We next determined whether IC exposure increased HSPC due to altered 137 intracellular iron availability or as a consequence of iron regulatory pathway activation.

138 Pharmacological inhibition of the limited iron response by employing a CD71-blocking 
139 antibody along with a highly specific inhibitor for lysosomal degradation of ferritin (Vps34i)

140 (Extended Data Fig. 4i) blocked the HSPC stimulatory effects of IC treatment (Fig. 1I),

141 as did heterozygous ablation of Fth1 (Extended Data Fig. 4j,k). This set of data

142 demonstrates that the increase in HSPC following IC-treatment is attributable to the

143 activation of the limited iron response which promptly replenishes the intracellular LIP

144 upon iron chelation.

145

146

147

148

149

150

151

152

153

154

155

156

157

158

159

160

161

\section{Fatty acid oxidation drives HSC expansion upon limited iron response activation}

To better understand the precise molecular mechanism leading to increased HSC numbers after iron homeostasis activation, we performed a comprehensive molecular analysis of primary HSC and HPC-7 cells following IC exposure. Compared to vehicle treated cells, IC exposure led to transcriptomic changes in HSC consistent with metabolic reprograming - affecting glycolysis as well as lipid, particularly arachidonic acid-driven, metabolism as early as 12 hrs and lasting for at least 48 hrs post treatment (Fig. 2a, Extended Data Fig. 5a-f, Extended Data Tables 2-5); we found alterations in steroid hormone receptor (peroxisome proliferator activated receptor (PPAR alpha) / retinoid X receptor (RXR alpha), Glutathione redox reactions and phosphatidylglycerol biosynthesis pathways (Extended Data Fig. 5e, Extended Data Table 4), associated with lipid metabolism and transport, amino acid metabolism and regulation of prostaglandin concentration (Extended Data Fig. 5f, Extended Data Tables 4,5). Metabolomic analysis of IC-treated HPC-7 cells as well as primary HSC-enriched cell populations confirmed these observations, and further suggested that the increase in fatty acid mobilization, lipolysis and fatty acid oxidation was at least in part dependent on

ferritinophagy (Extended Data Fig. 5g-j, Extended Data Tables 6-9). Direct 
162 quantification of fatty acid oxidation (FAO) showed increased abundance of a key FAO enzyme, Hadha, in primary HSC and other immature HPSC populations (Fig. 2b), as well as in HPC-7 cells (Extended Data Fig. 5k-m) upon IC exposure compared to mock

165 controls; we also found elevated basal and maximal FAO rates by Seahorse ${ }^{\mathrm{TM}}$ analysis

166 in hematopoietic Ba/F3 cells upon IC exposure of compared to vehicle controls, which

167 was reversible upon lipolysis blockage (Fig. 2c, Extended Data Fig. 5n,o). IC exposure 168 did not trigger detectable alterations in the overall ATP production while we noticed a 169 moderate, albeit statistically non-significant increase in FAO-driven ATP generation 170 (Extended Data Fig. 5p).

We tested whether increased FAO contributed to the stem cell stimulatory effects

172 of IC treatment. Pharmacological inhibition of mitochondrial FAO using carnitine

173 palmitoyltransferase-1 (CPT-1) inhibitor, etomoxir, prevented HSC expansion upon IC 174 exposure (Fig. 2d, Extended Data Fig. 5q). We also detected an increase in asymmetric 175 cell divisions driving IC-mediated HSC pool expansion (Fig. 2e), in line with prior work 176 demonstrating increased asymmetric cell divisions upon FAO stimulation in $\mathrm{HSC}^{50 .}$

177 Together, these data show that activation of the limited iron response triggers metabolic 178 reprogramming, increasing lipid mobilization and fatty acid oxidation which expands the 179 HSC population.

180 Arachidonic acid driven FAO drives iron dependent HSC expansion

181 We next investigated which fatty acid pool served as a source for the observed increase 182 in mitochondrial $\beta$-oxidation. We detected a transient increase in Cyclooxygenase 1 183 (COX-1 or prostaglandin G/H synthase 1/2 (Ptgs1)) in HSC 8 to 16 hrs upon IC treatment 184 compared to vehicle controls (Extended Data Fig. 6a), as well as elevated intracellular 
185 levels of the polyunsaturated fatty acid (PUFA) arachidonic acid (AA) (Fig. 2f). 186 Consistently, our molecular profiling showed alterations associated with increased AA 187 release, prostaglandin and eicosanoid production, as well as catabolism of AA (Fig. 2a, 188 Extended Data Fig. 5h,i). Analysis of proteome profiling data ${ }^{62}$ demonstrated the robust to high expression of several AA metabolic pathway enzymes in HSC; notably, enzymes

190 facilitating intracellular AA mobilization (Pla2g4a) and AA-dependent synthesis of 191 eicosanoids (Cyclooxygenases 1 and 2 (COX-1/2 or prostaglandin G/H synthase 1/2 192 (Ptgs1/2), Thromboxane A synthase 1 (platelet, cytochrome P450, family 5, subfamily A; 193 Tbxas1)) ${ }^{63}$ show particularly high protein levels in steady state HSC (Extended Data Fig. 194 6b), which is in line with the demonstrated regulatory function of AA derived lipid 195 metabolites and reactive oxygen species (ROS) formation in HSC self-renewal, homing 196 and engraftment ${ }^{64-66}$.

We also detected a prompt, yet transient increase in the abundance of Long-chain 198 fatty acid-CoA ligase 4 (Acsl4) upon IC exposure, which appeared dose dependent (Fig. 2g). Acsl4 is an essential acyl-CoA synthetase catalysing the conversion of long-chain 200 fatty acids, preferentially AA, into their active form, acyl-CoA, for lipid synthesis and 201 degradation via $\beta$-oxidation 67 . We predicted that increased Acs|4-mediated activation of 202 AA contributed to the elevated rates of FAO driving HSC expansion upon intracellular iron 203 chelation. RNAi mediated knock-down of Acs/4 (Extended Data Fig. 6c,d) reduced basal 204 FAO at steady state, as well as basal and maximal FAO upon IC treatment compared to 205 non-targeting controls (Fig. 2h, Extended Data Fig. 6e,f) in BaF/3 cells; importantly, 206 Acs/4 ablation also prevented IC-driven primary HSPC expansion (Fig. 2i). This set of 
207 results strongly supports a role for AA-driven $\beta$-oxidation in the IC-mediated increase of

208 functional stem cells.

209 Tip60 controls iron dependent gene expression programs in HSC

210

211

212

213

214

215

216

217

218

219

220

221

222

223

224

225

226

227

228

229

Lipid-derived acetyl-CoA can serve as a major carbon source for histone acetylation, especially when glucose and glutamine metabolism are impaired. ${ }^{68}$ Whether and how lipid carbon contributes to gene regulation in HSC has been unknown. Given the observed increase in FAO and concomitant decrease in glucose pathways following IC exposure (Fig. 2a-c, Extended Data Fig. 5a,c) ${ }^{44}$, we predicted that increased FAO would lead to functionally relevant changes in acetyl-CoA production and protein acetylation in HSC. In support, we found that addition of a pharmacologic inhibitor of ATP citrate lyase $(\mathrm{ACLY})$, a transferase that catalyses the conversion of citrate to acetyl-CoA in the cytoplasm and nucleus of cells, ${ }^{69}$ partially reversed the stem cell stimulatory effects of IC treatment (Fig. 3a, Extended Data Fig. 7a).

We furthermore found evidence of increased histone acetyltransferase activity, specifically MYST family member Tip60 (KAT5) - the acetyl-CoA N-acetyltransferase and catalytic subunit of the NuA4 complex upon IC treatment (Fig. 3b, Extended Data Fig.

7b,c). Tip60/NuA4 activates selected genes through acetylation of core histone $(\mathrm{H}) 4$ at lysines $(\mathrm{K})$ 5, 8 and $12, \mathrm{H} 2 \mathrm{~A}$ at $\mathrm{K} 5,{ }^{70,71}$ and histone variants $\mathrm{H} 2 \mathrm{AX}$ and $\mathrm{H} 2 \mathrm{AZ}{ }^{72}$. Tip60/KAT5 is an essential regulator of cellular homeostasis and stress response, and has been implicated in the maintenance and renewal of embryonic stem cells ${ }^{73}$ and $\mathrm{HSC}^{72}$. Using gene set enrichment analysis, we found a significant positive enrichment of H2AZac, H4K5ac, H4K8ac, H4K12ac, H4K16ac regulated genes at a genome wide level (Fig. 3c, Extended Data Fig. 7d). Interestingly, we also noted a strong enrichment of 
230

231

232

233

234

235

236

237

238

239

240

241

242

243

244

245

246

247

248

249

250

251

252

genes harbouring transcription activating mono- and tri methylation marks on $\mathrm{H} 3 \mathrm{~K} 4{ }^{74}$, as well as transcriptional fidelity-enhancing trimethylated $\mathrm{H} 3 \mathrm{~K}_{3} 6^{75}$ in $\mathrm{HSC}$ following IC treatment which appeared to be restricted to Tip60 occupied genes (Fig. 3c). These observations strongly suggested that IC treatment increases histone acetylation and enhances Tip60-mediated gene regulation in HSC.

Gene-specificity of the Tip60/NuA4 complex is conferred by several transcription factors, including $\mathrm{Myc}^{76}$ which is known to interact with the complex to drive $\mathrm{H} 3$ and 4 acetylation in mouse embryonic stem cells ${ }^{77}$, and $\mathrm{H} 2 \mathrm{AZ}$ acetylation in $\mathrm{LSK}^{\mathrm{HSSC}}{ }^{72}$. While we did not detect significant upregulation of $c-M y c$ in the majority of stem cells upon IC exposure (Extended Data Fig. 7e), we found enrichment of Myc-regulated genes, including known Tip60 targets differentially expressed (Extended Data Fig. 7b) and consistent with an increase in Myc activity in HSC following IC treatment (Extended Data

Fig. 7f-h). We furthermore uncovered enrichment of c-Myc regulated targets within the subset of direct Tip60 genes differentially expressed upon IC treatment in HSC (Fig. 3d, Extended Data Fig. 7i); analysis of transcriptional changes in HSC isolated from a mouse model of iron overload (induced by Fbx/5 deletion) ${ }^{43}$ further showed a large overlap of pathways shared with differential gene expression found in Myc or Tip60-deficient HSC (Extended Data Fig. 7j,k). This suggested that Tip60 and Myc cooperate in the regulation of gene expression following IC treatment of HSC. To test this, we evaluated whether inhibition of Tip60 and Myc would curtail the HSC stimulatory effects of IC. Fluidigm ${ }^{\mathrm{TM}}$ based gene expression assessment of a set of predicted Tip60/Myc regulated genes showed attenuation of several gene expression alterations upon IC exposure in HSC cotreated with highly selective pharmacologic inhibitors; these included Mevalonate kinase 
253 (Mvk) and Cyclin-dependent kinase 4 inhibitor D (Cdkn2d) (Fig. 3e), two targets of the

254 Mediator co-activator complex component CDK19 (Cyclin Dependent Kinase 19), a

255 negative regulator of lipogenesis and CD71 endocytosis ${ }^{78,79}$, and whose targets were

256 enriched in HSC-specific Tip60 regulated gene sets (Fig. 3d, Extended Data Fig. 7I).

257 Moreover, we confirmed upregulation of High Mobility Group AT-Hook 1 (Hmga1) (Fig.

258 3e), a chromatin modulator well expressed in $\mathrm{HSC}^{80}$, which is known to cooperate with

259 the Mediator complex to drive gene-specific regulation ${ }^{81}$, including the suppression of

$260 \mathrm{Numb}^{82}$ which induces differentiation in $\mathrm{HSC}^{25,83}$. At the cell functional level, 261 pharmacological inhibition of Tip60 or Myc showed a complete inhibition of the stem cell

262 stimulatory effects of IC while exerting no effect on steady state HSC (Fig. 3f,g, Extended

263 Data Fig. 7m). Together, these data show that iron limitation induces a gene-specific

264 increase in Tip60-mediated histone acetylation and enhanced gene expression control

265 orchestrated by Myc and likely CDK19/Mediator (Extended Data Fig. 7n,o) which

266 reinforces hematopoietic stem cell identity.

267 Cytoplasmic iron loading in ageing HSC

268 Loss of iron homoeostasis occurs during ageing and manifests in (1) iron deficiency 269 anaemia - originating from low circulating body iron due to insufficient iron consumption 270 or chronic inflammation ${ }^{84}$; (2) perturbed intracellular iron partition and mitochondrial

271 function caused by decreased iron sulphur cluster and heme biosynthesis ${ }^{85-89}$, as well as

272 (3) intracellular iron loading in several tissues ${ }^{90-94}$. We next assessed how ageing273 associated loss of iron homeostasis may affect tissue-specific stem cells.

275 well as $\mathrm{Fe}^{3+}$ pools in purified HSC from aged (22 to 24 mos.) vs. control cells from young 
276 (2 to 3 mos.) mice (Fig. 4a,b); a similar expansion of the LIP was detected in aged

277 multipotent and committed progenitor cells compared to young controls (Fig. 4a,b,

278 Extended Data Fig. 8a,b). Assessment of peripheral blood iron parameters showed no

279 signs of an overt systemic iron overload (Extended Data Fig. 8c,d). We next assessed whether an elevated LIP contributed to ageing-associated HSC dysfunction. treatment may reverse at least some of the ageing-associated phenotypes in HSC.

\section{Iron chelation restores function of aged HSC}

296 We next subjected old mice to a 14-day IC (or mock control) treatment regimen and, upon

297 its completion, competitively transplanted equal numbers of highly purified phenotypical

298 HSC into lethally irradiated young congenic recipients (Fig. 4j). Recipients of IC-treated 
299 donor stem cells showed an increase in overall donor cell chimerism (Fig. 4k) and an

300 increased number of donor-derived phenotypical HSC (Fig. 4I). Notably, we found a

301 concomitant decrease in phenotypic Mk-HSC with a known ageing-associated aberration

302 - cell surface presentation of glycoprotein (Gp) Ilb/llla integrin (CD41) (Fig. 4m), a

303 receptor for fibrinogen which is typically expressed on megakaryocytic (Mk) progenitors

304 and platelets ${ }^{100}$. In line, recipients of IC-treated aged HSC showed lower donor-derived

305 CD41 expressing Mk progenitor cell frequencies compared to mock treated control cell

306 recipients (Fig. 4n). Ex vivo IC treatment followed by quantification of megakaryopoiesis

307 commitment showed alleviation of the megakaryocytic differentiation bias of aged HSC

308 (Extended Fig. 8k) upon iron limitation (Fig. 40,p); notably, pharmacological inhibition of

309 Tip60 or c-Myc partially reversed the IC-induced mitigation of aberrantly increased

310 megakaryocytic differentiation bias of aged HSC (Fig. 4p, Extended Data Fig. 8I-n).

311 Together, these observations demonstrate that IC treatment can restore deregulated iron-

312 dependent molecular programs driving the dysfunction of aged stem cells.

\section{Discussion}

314 In this study, we provide evidence supporting the pool of redox active, readily accessible

315 labile iron as a key cellular rheostat allowing HSC to orchestrate metabolic and gene

316 regulatory pathways that reinforce stem cell identity. We also demonstrate that

317 cytoplasmic iron loading is a key factor in HSC dysfunction during ageing.

Intact iron homeostasis is known to ensure proper hematopoietic progenitor cell 
322 function (Extended Data Fig. 9): First, we uncovered that mitotic stem cells activate a

323 limited iron response during mitosis and upon experimental acute iron limitation. This

324 demonstrates that, like in metabolically highly active and proliferating cells ${ }^{46,102}$, the size

325 of the LIP is tightly regulated and closely linked with the functional state of quiescent and

326 metabolically less active stem cells ${ }^{26,49-52}$. It also shows that metabolic demands during

327 mitosis provide an effective trigger for the activation of iron homeostasis pathways in stem

328 cells. Second, our data show that the LIP serves as a molecular switch to transiently shift

329 heavily iron-dependent energy production ${ }^{103,104}$, including glycolysis (of quiescent stem

330

331

332

333

334

335

336

337

338

339

340

341

342

343

344

cells) and oxidative phosphorylation (of proliferating HSC), to metabolic pathways

requiring less iron - particularly fatty acid oxidation, which is known to expand the HSC pool $^{50}$. Third, our data strongly suggest increased lipid carbon-fuelled histone acetylation and epigenetic regulation to follow iron homeostasis pathway activation. This finding strengthens the emerging paradigm of a tight interconnection between lipid metabolism and epigenetic control in stem cells ${ }^{105,106 ;}$ it also provides mechanistic insights into metabolism-driven epigenetic regulation of HSC identity. Fourth, we demonstrate that cytoplasmic iron loading occurs in aged stem cells, blunting iron-dependent HSC gene regulation, and driving the dysfunction of aged stem cells.

Given that many somatic stem cells share principle cellular and molecular regulatory circuits $^{107}$, it is possible that LIP serves as a general rheostat in various adult stem cells. As loss of iron homeostasis is observed in a large fraction of the elderly ${ }^{108}$, in patients with chronic inflammation ${ }^{109}$ or cancer $^{110}$, our findings will have implications in understanding and therapeutic mitigation of altered stem cell function in a wide range of degenerative and malignant pathologies. 


\section{Main References}

1 Till, J. E., McCulloch, E. A. \& Siminovitch, L. A Stochastic Model of Stem Cell Proliferation, Based on the Growth of Spleen Colony-Forming Cells. Proc Natl Acad Sci U S A 51, 29-36 (1964).

2 Spangrude, G. J., Heimfeld, S. \& Weissman, I. L. Purification and characterization of mouse hematopoietic stem cells. Science 241, 58-62 (1988).

3 Suda, T., Suda, J. \& Ogawa, M. Proliferative kinetics and differentiation of murine blast cell colonies in culture: evidence for variable G0 periods and constant doubling rates of early pluripotent hemopoietic progenitors. J Cell Physiol 117, 308-318 (1983).

4 Suda, T., Suda, J. \& Ogawa, M. Single-cell origin of mouse hemopoietic colonies expressing multiple lineages in variable combinations. Proc Natl Acad Sci U S A 80, 66896693 (1983).

5 Baum, C. M., Weissman, I. L., Tsukamoto, A. S., Buckle, A. M. \& Peault, B. Isolation of a candidate human hematopoietic stem-cell population. Proc Natl Acad Sci U S A 89, 2804-2808 (1992).

6 Morrison, S. J., Wandycz, A. M., Akashi, K., Globerson, A. \& Weissman, I. L. The aging of hematopoietic stem cells. Nat Med 2, 1011-1016 (1996).

7 de Haan, G. \& Lazare, S. S. Aging of hematopoietic stem cells. Blood 131, 479-487 (2018).

8 Will, B. et al. Minimal PU.1 reduction induces a preleukemic state and promotes development of acute myeloid leukemia. Nature medicine 21, 1172-1181 (2015).

9 Dong, S. et al. Chaperone-mediated autophagy sustains haematopoietic stem-cell function. Nature 591, 117-123 (2021).

10 Jacobs, A. An intracellular transit iron pool. Ciba Found Symp, 91-106 (1976).

11 Orford, K. W. \& Scadden, D. T. Deconstructing stem cell self-renewal: genetic insights into cell-cycle regulation. Nat Rev Genet 9, 115-128 (2008).

12 Arai, F. \& Suda, T. Maintenance of quiescent hematopoietic stem cells in the osteoblastic niche. Ann N Y Acad Sci 1106, 41-53 (2007).

13 Cheng, T. et al. Hematopoietic stem cell quiescence maintained by p21cip1/waf1. Science 287, 1804-1808 (2000).

14 Wilson, A. et al. Hematopoietic stem cells reversibly switch from dormancy to selfrenewal during homeostasis and repair. Cell 135, 1118-1129 (2008).

15 Busque, L. et al. Nonrandom X-inactivation patterns in normal females: Iyonization ratios vary with age. Blood $\mathbf{8 8}$, 59-65 (1996).

16 Busque, L. et al. Recurrent somatic TET2 mutations in normal elderly individuals with clonal hematopoiesis. Nat Genet 44, 1179-1181 (2012).

17 Maryanovich, M. et al. Adrenergic nerve degeneration in bone marrow drives aging of the hematopoietic stem cell niche. Nature medicine 24, 782-791 (2018).

18 Kusumbe, A. P. et al. Age-dependent modulation of vascular niches for haematopoietic stem cells. Nature 532, 380-384 (2016).

19 Verovskaya, E. V., Dellorusso, P. V. \& Passegue, E. Losing Sense of Self and Surroundings: Hematopoietic Stem Cell Aging and Leukemic Transformation. Trends Mol Med 25, 494515 (2019). 
38820 Beerman, l. et al. Functionally distinct hematopoietic stem cells modulate

389 hematopoietic lineage potential during aging by a mechanism of clonal expansion. Proceedings of the National Academy of Sciences of the United States of America 107, 5465-5470 (2010).

21 Fuster, J. J. et al. Clonal hematopoiesis associated with TET2 deficiency accelerates atherosclerosis development in mice. Science 355, 842-847 (2017).

22 Corces-Zimmerman, M. R., Hong, W. J., Weissman, I. L., Medeiros, B. C. \& Majeti, R. Preleukemic mutations in human acute myeloid leukemia affect epigenetic regulators and persist in remission. Proceedings of the National Academy of Sciences of the United States of America 111, 2548-2553 (2014).

23 Mayani, H., Dragowska, W. \& Lansdorp, P. M. Lineage commitment in human hemopoiesis involves asymmetric cell division of multipotent progenitors and does not appear to be influenced by cytokines. J Cell Physiol 157, 579-586 (1993). Takano, H., Ema, H., Sudo, K. \& Nakauchi, H. Asymmetric division and lineage commitment at the level of hematopoietic stem cells: inference from differentiation in daughter cell and granddaughter cell pairs. J Exp Med 199, 295-302 (2004).

404 Loeffler, D. et al. Asymmetric lysosome inheritance predicts activation of haematopoietic stem cells. Nature 573, 426-429 (2019). Simsek, T. et al. The distinct metabolic profile of hematopoietic stem cells reflects their location in a hypoxic niche. Cell Stem Cell 7, 380-390 (2010). Mortensen, M. et al. The autophagy protein Atg7 is essential for hematopoietic stem cell maintenance. J Exp Med 208, 455-467 (2011). Tothova, Z. et al. FoxOs are critical mediators of hematopoietic stem cell resistance to physiologic oxidative stress. Cell 128, 325-339 (2007).

413

29 Ho, T. T. et al. Autophagy maintains the metabolism and function of young and old stem cells. Nature 543, 205-210 (2017). embryonic stem cell self-renewal and differentiation. Cell Stem Cell 2, 448-460 (2008).

31 Signer, R. A. et al. The rate of protein synthesis in hematopoietic stem cells is limited partly by 4E-BPs. Genes Dev 30, 1698-1703 (2016). Regulating Muscle Stem Cell Quiescence and Self-Renewal. Cell Stem Cell 18, 79-90 (2016). synthesis. Nature 534, 335-340 (2016). sequence-specific transcription factors from mitotic chromatin. Cell 83, 29-38 (1995). Wang, Z. et al. Cyclin B1/Cdk1 coordinates
progression. Dev Cell 29, 217-232 (2014).

428 Palozola, K. C. et al. Mitotic transcription and waves of gene reactivation during mitotic exit. Science 358, 119-122 (2017).

37 Javasky, E. et al. Study of mitotic chromatin supports a model of bookmarking by histone modifications and reveals nucleosome deposition patterns. Genome Res 28, 1455-1466 (2018). 
43238 Vander Heiden, M. G., Cantley, L. C. \& Thompson, C. B. Understanding the Warburg

433

434

435

436

437

438

439

440

441

442

443

444

445

446

447

448

449

450

451

452

453

454

455

456

457

458

459

460

461

462

463

464

465

466

467

468

469

470

471

472

473

474

effect: the metabolic requirements of cell proliferation. Science 324, 1029-1033 (2009).

39 Aisen, P., Enns, C. \& Wessling-Resnick, M. Chemistry and biology of eukaryotic iron metabolism. Int J Biochem Cell Biol 33, 940-959 (2001).

40 Rothman, R. J., Serroni, A. \& Farber, J. L. Cellular pool of transient ferric iron, chelatable by deferoxamine and distinct from ferritin, that is involved in oxidative cell injury. $\mathrm{Mol}$ Pharmacol 42, 703-710 (1992).

41 Kruszewski, M. Labile iron pool: the main determinant of cellular response to oxidative stress. Mutat Res 531, 81-92 (2003).

42 Crichton, R. R., Wilmet, S., Legssyer, R. \& Ward, R. J. Molecular and cellular mechanisms of iron homeostasis and toxicity in mammalian cells. J Inorg Biochem 91, 9-18 (2002).

43 Muto, Y., Nishiyama, M., Nita, A., Moroishi, T. \& Nakayama, K. I. Essential role of FBXL5mediated cellular iron homeostasis in maintenance of hematopoietic stem cells. Nat Commun 8, 16114 (2017).

44 Kao, Y. R. et al. Thrombopoietin receptor-independent stimulation of hematopoietic stem cells by eltrombopag. Science translational medicine 10 (2018).

45 Breuer, W., Epsztejn, S. \& Cabantchik, Z. I. Iron acquired from transferrin by K562 cells is delivered into a cytoplasmic pool of chelatable iron(II). J Biol Chem 270, 24209-24215 (1995).

46 Breuer, W., Epsztejn, S. \& Cabantchik, Z. I. Dynamics of the cytosolic chelatable iron pool of K562 cells. FEBS Lett 382, 304-308 (1996).

47 Oguro, H., Ding, L. \& Morrison, S. J. SLAM family markers resolve functionally distinct subpopulations of hematopoietic stem cells and multipotent progenitors. Cell stem cell 13, 102-116 (2013).

48 Kiel, M. J. et al. SLAM family receptors distinguish hematopoietic stem and progenitor cells and reveal endothelial niches for stem cells. Cell 121, 1109-1121 (2005).

49 Takubo, K. et al. Regulation of the HIF-1alpha level is essential for hematopoietic stem cells. Cell Stem Cell 7, 391-402 (2010).

50 Ito, K. et al. A PML-PPAR-delta pathway for fatty acid oxidation regulates hematopoietic stem cell maintenance. Nat Med 18, 1350-1358 (2012).

51 Takubo, K. et al. Regulation of Glycolysis by Pdk Functions as a Metabolic Checkpoint for Cell Cycle Quiescence in Hematopoietic Stem Cells. Cell stem cell 12, 49-61 (2013).

52 Maryanovich, M. et al. An $\mathrm{MTCH} 2$ pathway repressing mitochondria metabolism regulates haematopoietic stem cell fate. Nat Commun 6, 7901 (2015).

53 Beckmann, J., Scheitza, S., Wernet, P., Fischer, J. C. \& Giebel, B. Asymmetric cell division within the human hematopoietic stem and progenitor cell compartment: identification of asymmetrically segregating proteins. Blood 109, 5494-5501 (2007).

54 Hentze, M. W. et al. Identification of the iron-responsive element for the translational regulation of human ferritin mRNA. Science 238, 1570-1573 (1987).

55 Anderson, S. A. et al. The IRP1-HIF-2alpha axis coordinates iron and oxygen sensing with erythropoiesis and iron absorption. Cell Metab 17, 282-290 (2013).

56 Wilkinson, N. \& Pantopoulos, K. IRP1 regulates erythropoiesis and systemic iron homeostasis by controlling HIF2alpha mRNA translation. Blood 122, 1658-1668 (2013). 
$47557 \quad$ Ghosh, M. C. et al. Deletion of iron regulatory protein 1 causes polycythemia and pulmonary hypertension in mice through translational derepression of HIF2alpha. Cell Metab 17, 271-281 (2013).

58 Wheat, J. C. et al. Single-molecule imaging of transcription dynamics in somatic stem cells. Nature 583, 431-436 (2020).

59 Mancias, J. D., Wang, X., Gygi, S. P., Harper, J. W. \& Kimmelman, A. C. Quantitative proteomics identifies NCOA4 as the cargo receptor mediating ferritinophagy. Nature 509, 105-109 (2014).

60 Glickstein, H. et al. Action of chelators in iron-loaded cardiac cells: Accessibility to intracellular labile iron and functional consequences. Blood 108, 3195-3203 (2006).

61 Glickstein, H., El, R. B., Shvartsman, M. \& Cabantchik, Z. I. Intracellular labile iron pools as direct targets of iron chelators: a fluorescence study of chelator action in living cells. Blood 106, 3242-3250 (2005).

62 Haas, S. et al. Inflammation-Induced Emergency Megakaryopoiesis Driven by Hematopoietic Stem Cell-like Megakaryocyte Progenitors. Cell Stem Cell 17, 422-434 (2015).

63 Funk, C. D. Prostaglandins and leukotrienes: advances in eicosanoid biology. Science 294, 1871-1875 (2001).

$64 \mathrm{Li}, \mathrm{P}$. et al. Epoxyeicosatrienoic acids enhance embryonic haematopoiesis and adult marrow engraftment. Nature 523, 468-471 (2015).

65 Kinder, M. et al. Hematopoietic stem cell function requires 12/15-lipoxygenasedependent fatty acid metabolism. Blood 115, 5012-5022 (2010).

66 Desplat, V. et al. Effects of lipoxygenase metabolites of arachidonic acid on the growth of human blood CD34(+) progenitors. Blood Cells Mol Dis 26, 427-436 (2000).

67 Golej, D. L. et al. Long-chain acyl-CoA synthetase 4 modulates prostaglandin $\mathrm{E}(2)$ release from human arterial smooth muscle cells. J Lipid Res 52, 782-793 (2011).

68 McDonnell, E. et al. Lipids Reprogram Metabolism to Become a Major Carbon Source for Histone Acetylation. Cell Rep 17, 1463-1472 (2016).

69 Pearce, N. J. et al. The role of ATP citrate-lyase in the metabolic regulation of plasma lipids. Hypolipidaemic effects of SB-204990, a lactone prodrug of the potent ATP citratelyase inhibitor SB-201076. The Biochemical journal 334 ( Pt 1), 113-119 (1998).

70 Galarneau, L. et al. Multiple links between the NuA4 histone acetyltransferase complex and epigenetic control of transcription. Mol Cell 5, 927-937 (2000).

71 Krogan, N. J. et al. Regulation of chromosome stability by the histone H2A variant Htz1, the Swr1 chromatin remodeling complex, and the histone acetyltransferase NuA4. Proc Natl Acad Sci U S A 101, 13513-13518 (2004).

72 Numata, A. et al. Lysine acetyltransferase Tip60 is required for hematopoietic stem cell maintenance. Blood (2020).

73 Fazzio, T. G., Huff, J. T. \& Panning, B. An RNAi screen of chromatin proteins identifies Tip60-p400 as a regulator of embryonic stem cell identity. Cell 134, 162-174 (2008).

74 Krogan, N. J. et al. The Paf1 complex is required for histone H3 methylation by COMPASS and Dot1p: linking transcriptional elongation to histone methylation. Mol Cell 11, 721729 (2003). 
$51875 \quad$ Sen, P. et al. H3K36 methylation promotes longevity by enhancing transcriptional

$519 \quad$ fidelity. Genes Dev 29, 1362-1376 (2015).

52076 Allard, S. et al. NuA4, an essential transcription adaptor/histone $\mathrm{H} 4$ acetyltransferase

521

522

523

524

525

526

527

528

529

530

531

532

533

534

535

536

537

538

539

540

541

542

543

544

545

546

547

548

549

550

551

552

553

554

555

556

$557 \quad 92$

558

559

560 complex containing Esa1p and the ATM-related cofactor Tra1p. EMBO J 18, 5108-5119 (1999).

77 Kim, J. et al. A Myc network accounts for similarities between embryonic stem and cancer cell transcription programs. Cell 143, 313-324 (2010).

78 Poss, Z. C. et al. Identification of Mediator Kinase Substrates in Human Cells using Cortistatin A and Quantitative Phosphoproteomics. Cell Rep 15, 436-450 (2016).

79 Collinet, C. et al. Systems survey of endocytosis by multiparametric image analysis. Nature 464, 243-249 (2010).

80 Cabezas-Wallscheid, N. et al. Identification of regulatory networks in HSCs and their immediate progeny via integrated proteome, transcriptome, and DNA methylome analysis. Cell Stem Cell 15, 507-522 (2014).

$81 \mathrm{Xu}, \mathrm{M}$. et al. Core promoter-selective function of HMGA1 and Mediator in Initiatordependent transcription. Genes Dev 25, 2513-2524 (2011).

82 Puca, F. et al. HMGA1 negatively regulates NUMB expression at transcriptional and post transcriptional level in glioblastoma stem cells. Cell Cycle 18, 1446-1457 (2019).

$83 \mathrm{Wu}, \mathrm{M}$. et al. Imaging hematopoietic precursor division in real time. Cell Stem Cell 1, 541-554 (2007).

84 Eisenstaedt, R., Penninx, B. W. \& Woodman, R. C. Anemia in the elderly: current understanding and emerging concepts. Blood Rev 20, 213-226 (2006).

85 Veatch, J. R., McMurray, M. A., Nelson, Z. W. \& Gottschling, D. E. Mitochondrial dysfunction leads to nuclear genome instability via an iron-sulfur cluster defect. Cell 137, 1247-1258 (2009).

86 Bitar, M. \& Weiner, M. Modification of age-induced changes in heme and hemoproteins by testosterone in male rats. Mech Ageing Dev 23, 285-296 (1983).

87 Atamna, H., Liu, J. \& Ames, B. N. Heme deficiency selectively interrupts assembly of mitochondrial complex IV in human fibroblasts: revelance to aging. The Journal of biological chemistry 276, 48410-48416 (2001).

88 Atamna, H., Killilea, D. W., Killilea, A. N. \& Ames, B. N. Heme deficiency may be a factor in the mitochondrial and neuronal decay of aging. Proceedings of the National Academy of Sciences of the United States of America 99, 14807-14812 (2002).

89 Hughes, C. E. et al. Cysteine Toxicity Drives Age-Related Mitochondrial Decline by Altering Iron Homeostasis. Cell 180, 296-310 e218 (2020).

90 Doulias, P. T. et al. Flow cytometric estimation of 'labile iron pool' in human white blood cells reveals a positive association with ageing. Free Radic Res 42, 253-259 (2008).

91 Hahn, P. et al. Age-dependent and gender-specific changes in mouse tissue iron by strain. Exp Gerontol 44, 594-600 (2009).

92 Hallgren, B. \& Sourander, P. The effect of age on the non-haemin iron in the human brain. J Neurochem 3, 41-51 (1958).

93 Pankhurst, Q., Hautot, D., Khan, N. \& Dobson, J. Increased levels of magnetic iron compounds in Alzheimer's disease. J Alzheimers Dis 13, 49-52 (2008). 
56194 Xu, J., Knutson, M. D., Carter, C. S. \& Leeuwenburgh, C. Iron accumulation with age,

562

563

564

565

566

567

568

569

570

571

572

573

574

575

576

577

578

579

580

581

582

583

584

585

586

587

588

589

590

591

592

593

594

595

596

597

598

599

oxidative stress and functional decline. PloS one 3, e2865 (2008).

95 Svendsen, A. F. et al. A comprehensive transcriptome signature of murine hematopoietic stem cell aging. Blood (2021).

96 Sathyan, S. et al. Plasma proteomic profile of age, health span, and all-cause mortality in older adults. Aging Cell 19, e13250 (2020).

97 Adelman, E. R. et al. Aging Human Hematopoietic Stem Cells Manifest Profound Epigenetic Reprogramming of Enhancers That May Predispose to Leukemia. Cancer Discov 9, 1080-1101 (2019).

98 Grover, A. et al. Single-cell RNA sequencing reveals molecular and functional platelet bias of aged haematopoietic stem cells. Nat Commun 7, 11075 (2016).

99 Rodriguez-Fraticelli, A. E. et al. Clonal analysis of lineage fate in native haematopoiesis. Nature 553, 212-+ (2018).

100 Gekas, C. \& Graf, T. CD41 expression marks myeloid-biased adult hematopoietic stem cells and increases with age. Blood 121, 4463-4472 (2013).

101 Broxmeyer, H. E., Cooper, S., Levi, S. \& Arosio, P. Mutated recombinant human heavychain ferritins and myelosuppression in vitro and in vivo: a link between ferritin ferroxidase activity and biological function. Proc Natl Acad Sci U S A 88, 770-774 (1991).

102 Konijn, A. M. et al. The cellular labile iron pool and intracellular ferritin in K562 cells. Blood 94, 2128-2134 (1999).

103 Oexle, H., Gnaiger, E. \& Weiss, G. Iron-dependent changes in cellular energy metabolism: influence on citric acid cycle and oxidative phosphorylation. Biochim Biophys Acta 1413, 99-107 (1999).

104 Keller, M. A. et al. Conditional iron and pH-dependent activity of a non-enzymatic glycolysis and pentose phosphate pathway. Sci Adv 2, e1501235 (2016).

105 Moussaieff, A. et al. Glycolysis-mediated changes in acetyl-CoA and histone acetylation control the early differentiation of embryonic stem cells. Cell Metab 21, 392-402 (2015).

106 Ryall, J. G. et al. The NAD(+)-dependent SIRT1 deacetylase translates a metabolic switch into regulatory epigenetics in skeletal muscle stem cells. Cell Stem Cell 16, 171-183 (2015).

107 Slack, J. M. Origin of stem cells in organogenesis. Science 322, 1498-1501 (2008).

108 Guralnik, J. M., Eisenstaedt, R. S., Ferrucci, L., Klein, H. G. \& Woodman, R. C. Prevalence of anemia in persons 65 years and older in the United States: evidence for a high rate of unexplained anemia. Blood 104, 2263-2268 (2004).

109 Nemeth, E. \& Ganz, T. Anemia of inflammation. Hematol Oncol Clin North Am 28, 671681, vi (2014).

110 Ludwig, H. et al. The European Cancer Anaemia Survey (ECAS): a large, multinational, prospective survey defining the prevalence, incidence, and treatment of anaemia in cancer patients. Eur J Cancer 40, 2293-2306 (2004). 
bioRxiv preprint doi: https://doi.org/10.1101/2021.08.03.454947; this version posted August 5, 2021. The copyright holder for this preprint (which was not certified by peer review) is the author/funder, who has granted bioRxiv a license to display the preprint in perpetuity. It is made available under aCC-BY-NC-ND 4.0 International license.

Figure 1

a Stem Cell Signature

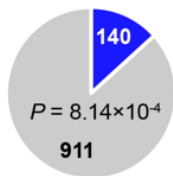

With IRE $\square$ Without IRE

e

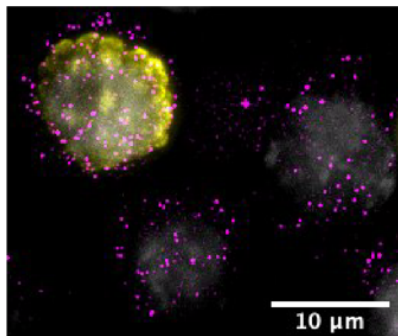

b

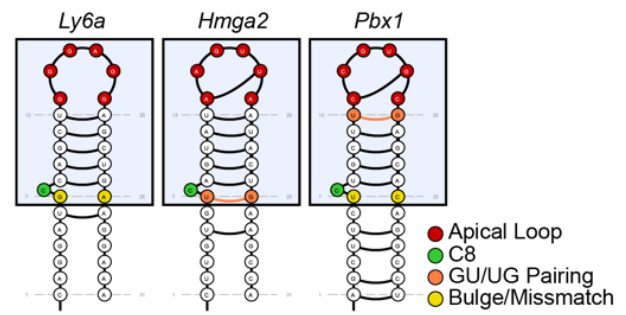

C

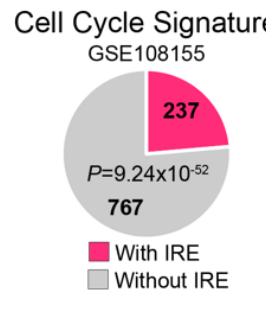

d

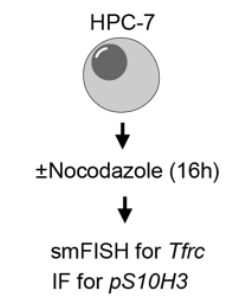

h
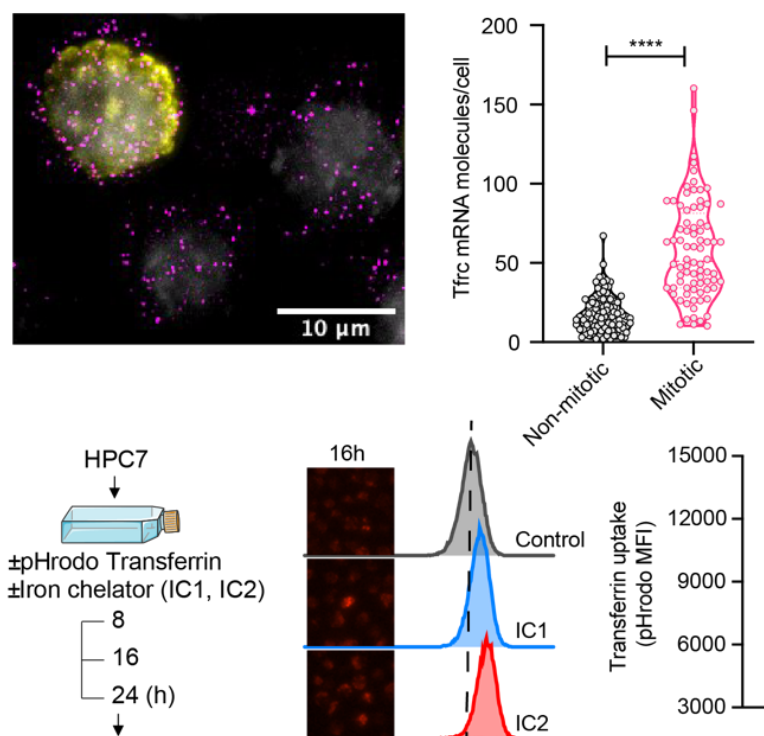

f

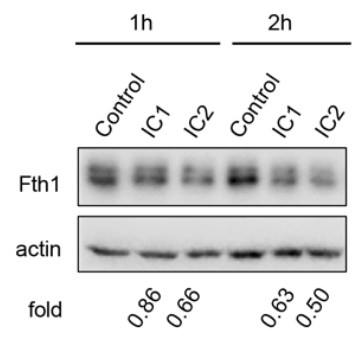

i

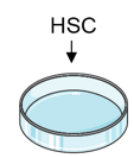

\pm Iron chelator $(48 \mathrm{~h})$

Single Cell RNA-seq

Phenotypic Analysis (HIf, Ly6a)

HSC

NonHSC

I

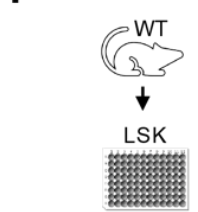

\pm Iron chelator (IC,48h) \pm Rescue $(48 \mathrm{~h})[$ anti-CD7

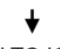
LTC-IC
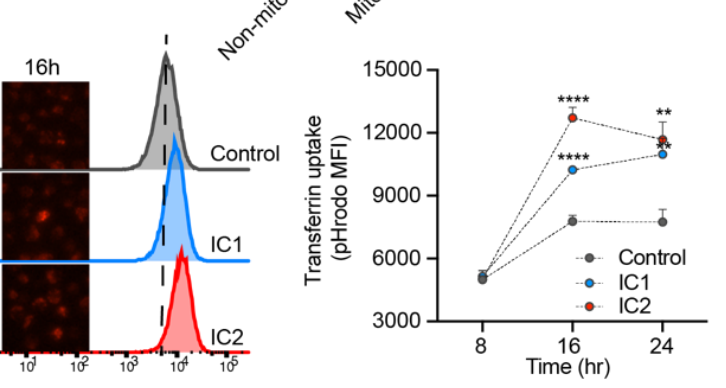

j

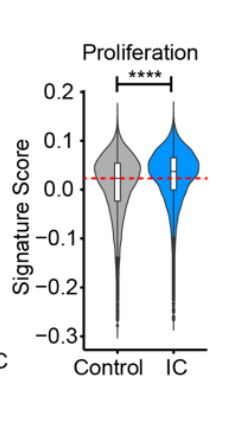

k

Control

$34.5 \% \mathrm{HSC}$

$65.5 \%$ NonHSC

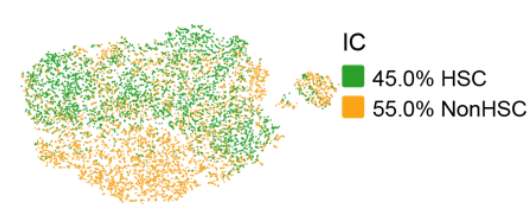

IC

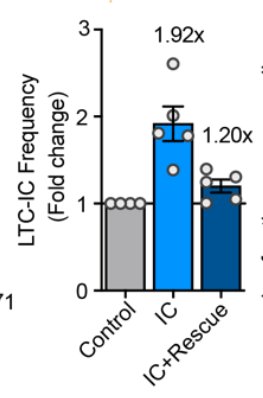

g
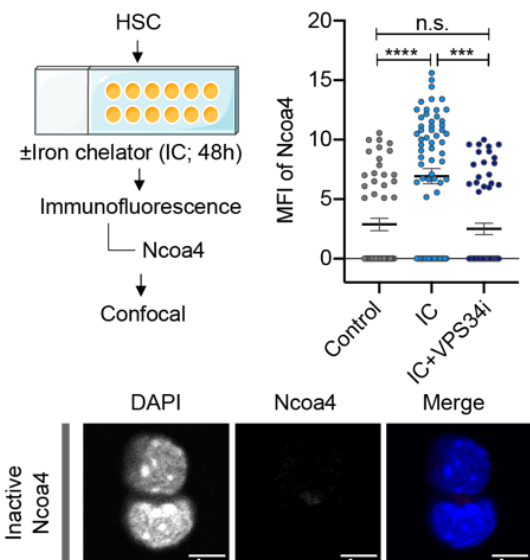

Merge

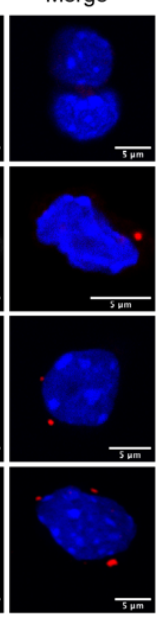


bioRxiv preprint doi: https://doi.org/10.1101/2021.08.03.454947; this version posted Auqust 5, 2021. The copyright holder for this preprint (which was not certified by peer review) is the author/funder, who has granted bioRxiv a license to display the preprint in perpetuity. It is made available under aCC-BY-NC-ND 4.0 International license.

Figure 2

a

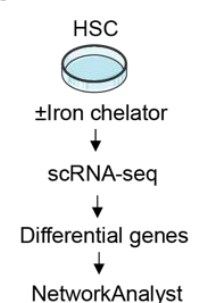

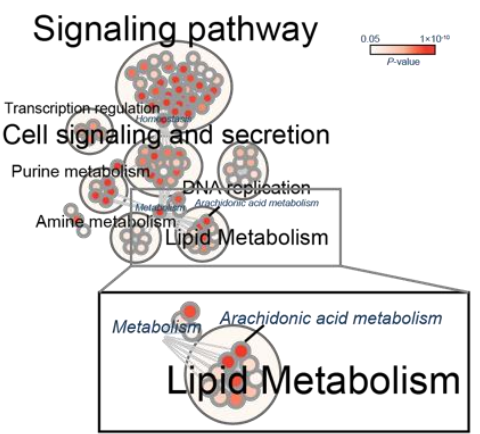

b

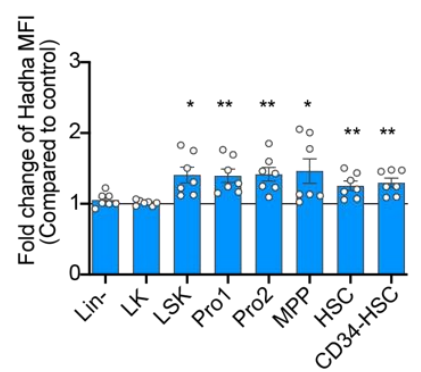

C

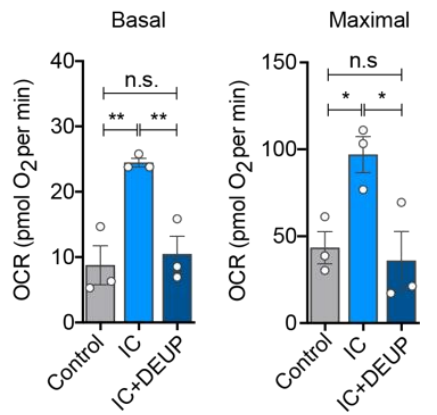

d

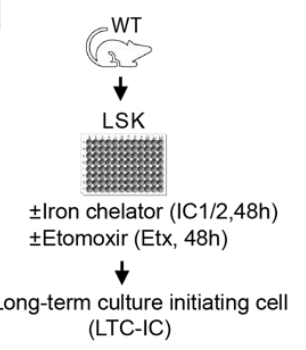

f

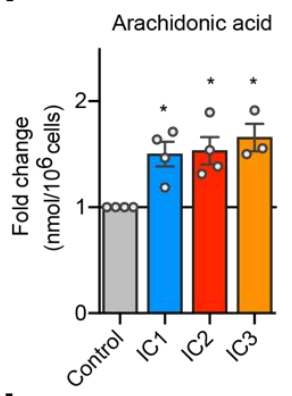

h

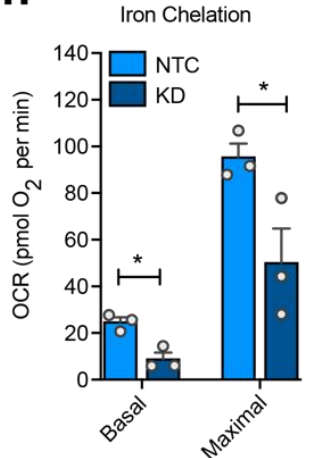

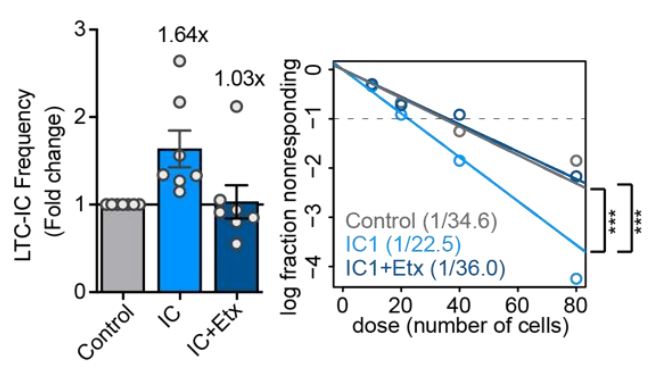

g

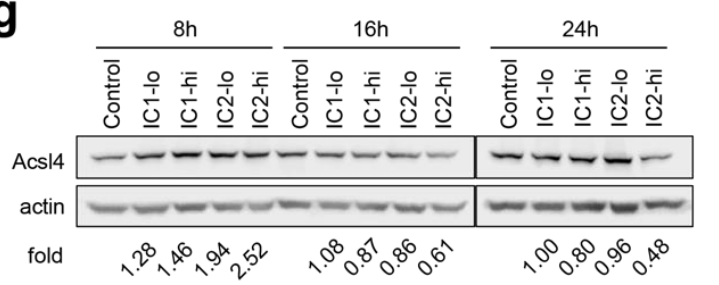

i

cKit+ BM cells

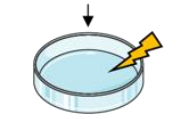

Acsl4 knockdown

— Targeted (KD)

- NTC

$\downarrow$

IIron chelator (IC, 48h)

LTC-IC
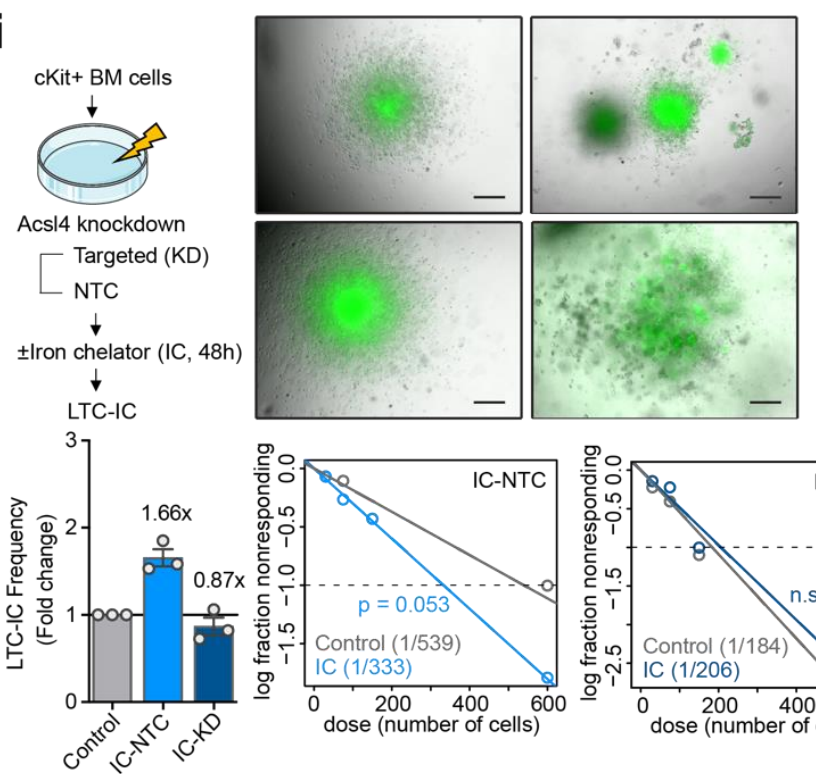

e
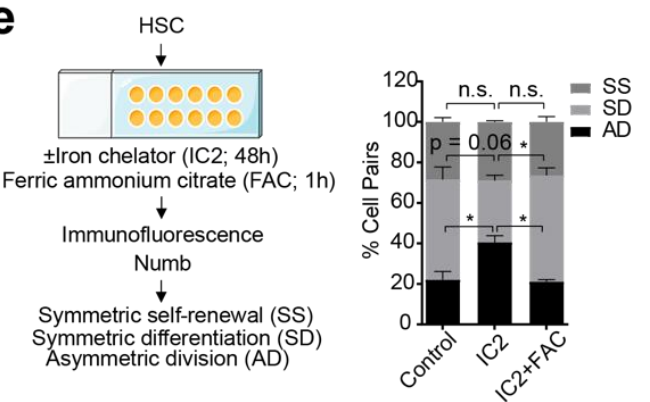

Numb Merge

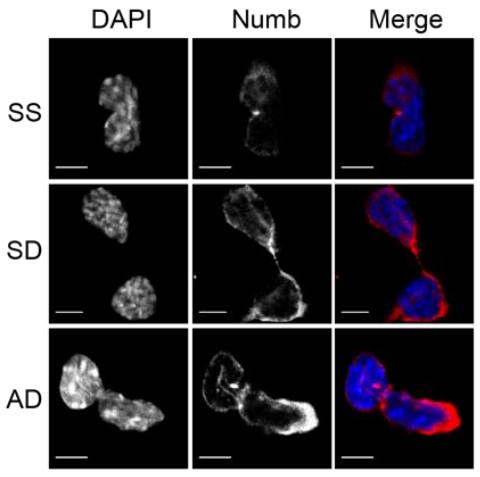

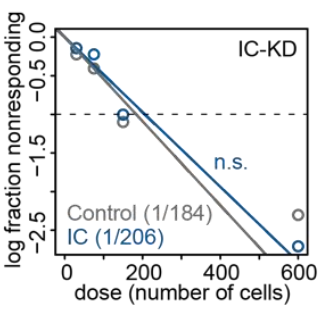


bioRxiv preprint doi: https://doi.org/10.1101/2021.08.03.454947; this version posted Auqust 5, 2021. The copyright holder for this preprint (which was not certified by peer review) is the author/funder, who has granted bioRxiv a license to display the preprint in perpetuity. It is made available under aCC-BY-NC-ND 4.0 International license.

Figure 3

a

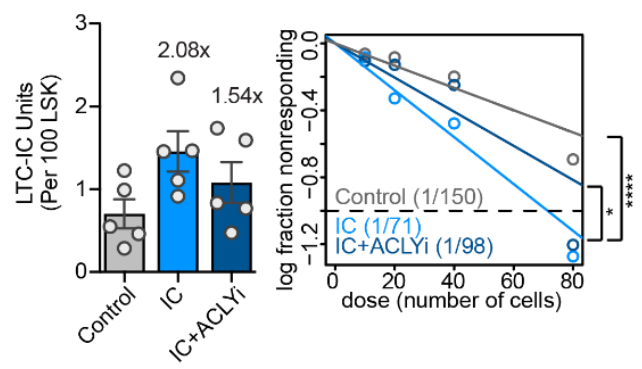

C

$\mathbf{e}$
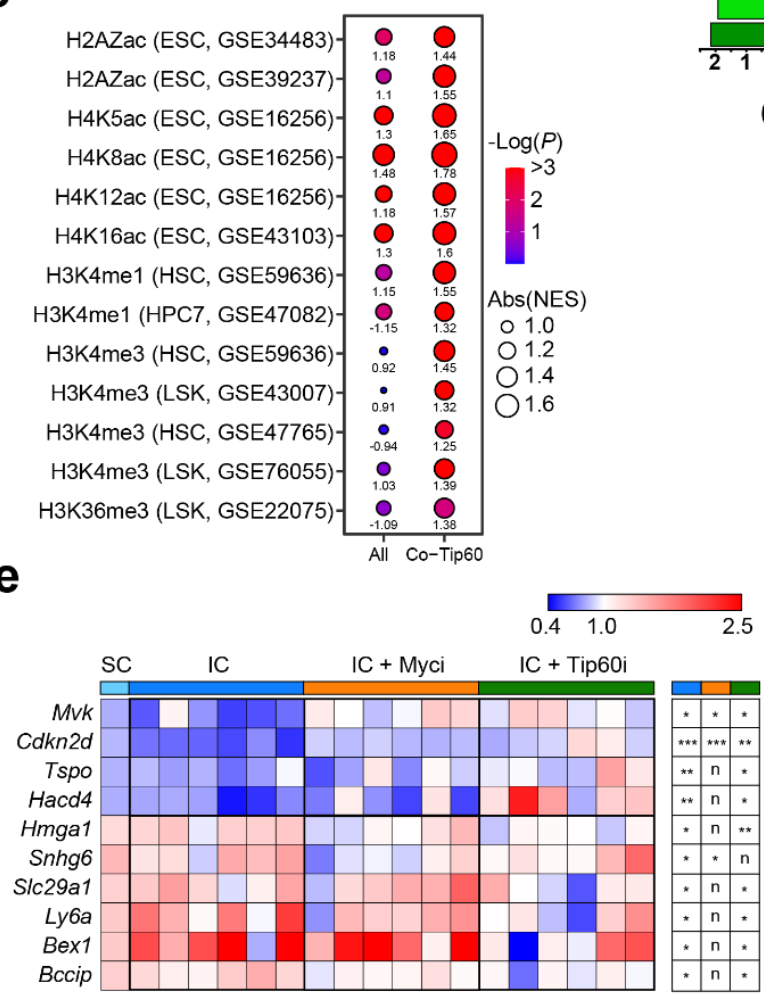

g

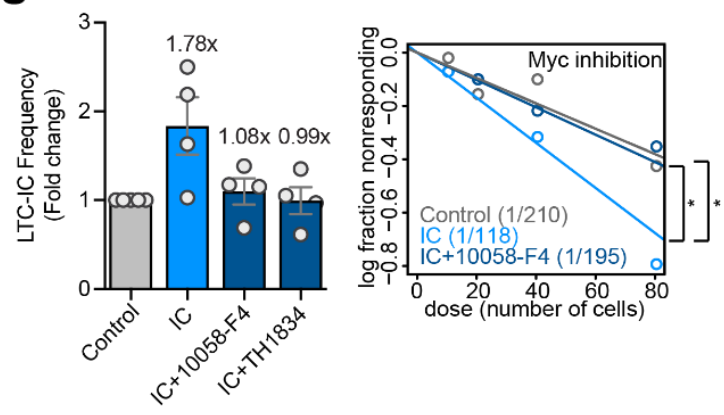

b $\quad$ TIP60 KO UP $(P<0.001)$
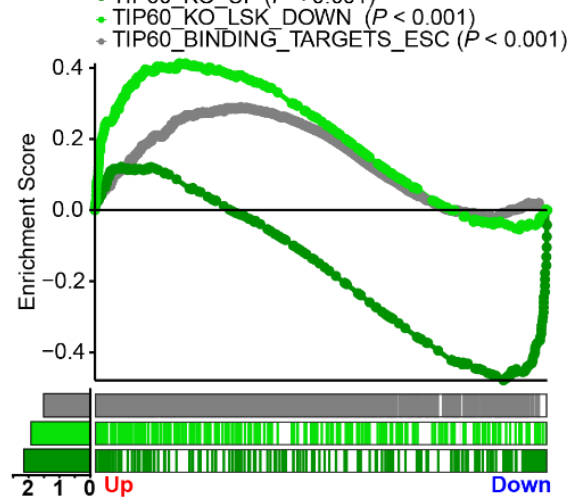

210 Up

d

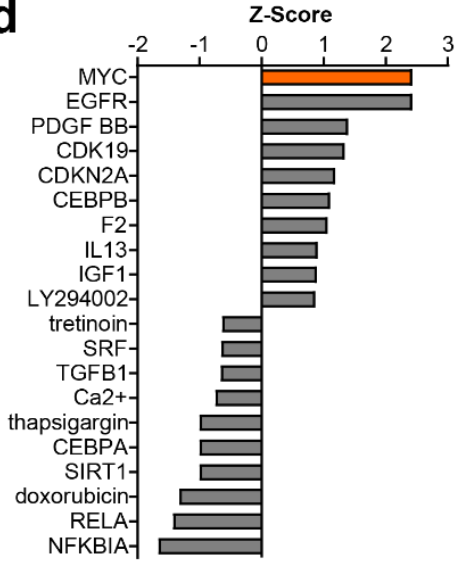

f

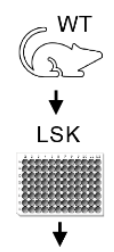

IIron chelator (IC,48h)

\pm Rescue (48h) $10058-\mathrm{F} 4$ (Myci)

$\downarrow$ TH1834 (Tip60i)

LTC-IC

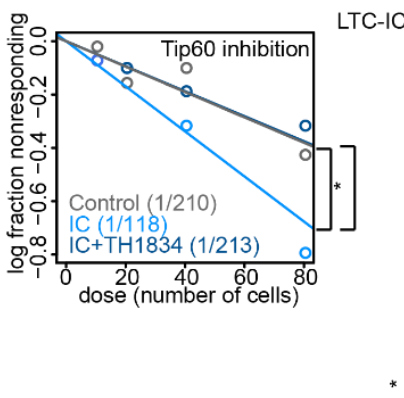


bioRxiv preprint doi: https://doi.org/10.1101/2021.08.03.454947; this version posted Auqust 5, 2021. The copyright holder for this preprint (which was not certified by peer review) is the author/funder, who has granted bioRxiv a license to display the preprint in perpetuity. It is made available under aCC-BY-NC-ND 4.0 International license.

a

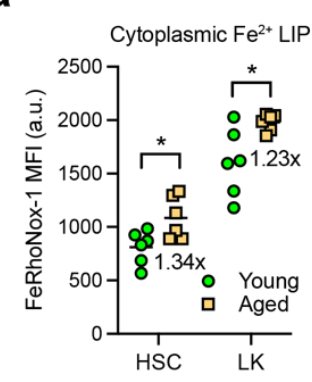

b

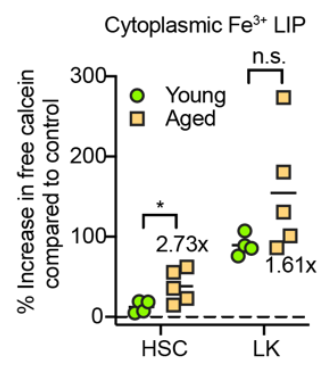

C

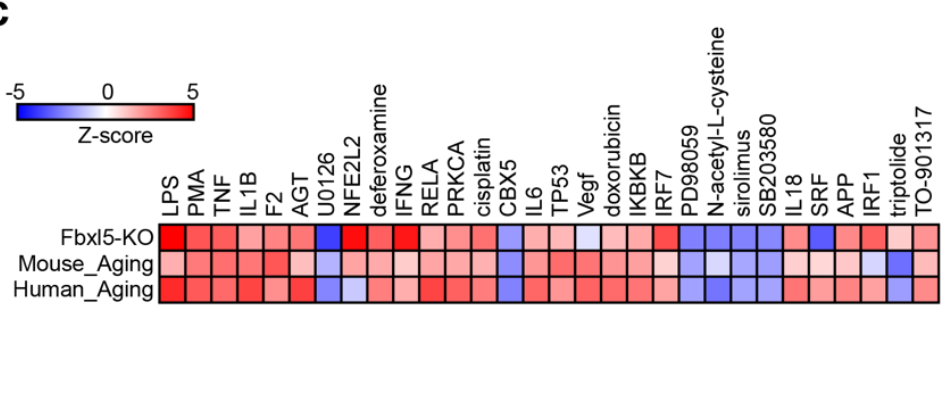

f

Up in

aged HSC

Down in
aged HSC

g

h Aging Signature
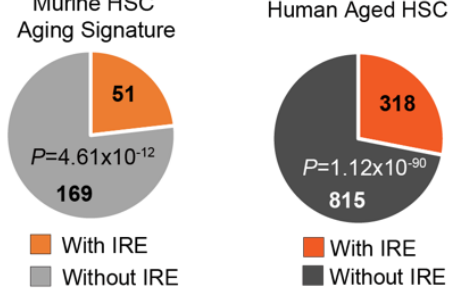

- REGULATION_OF_MK DIFFERENTIATION $(P<0.001)$ - UP_AGED_VS_YOUNG_VWF+HSC $(P<0.001)$

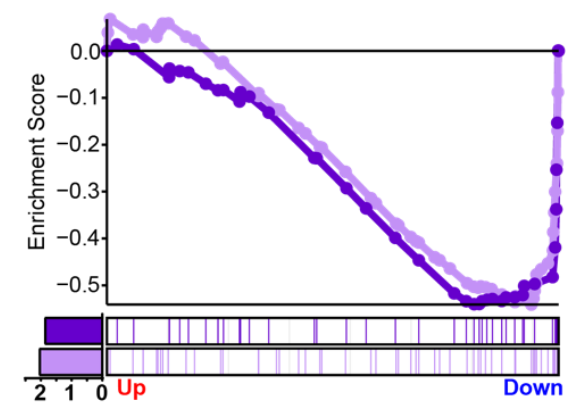

k

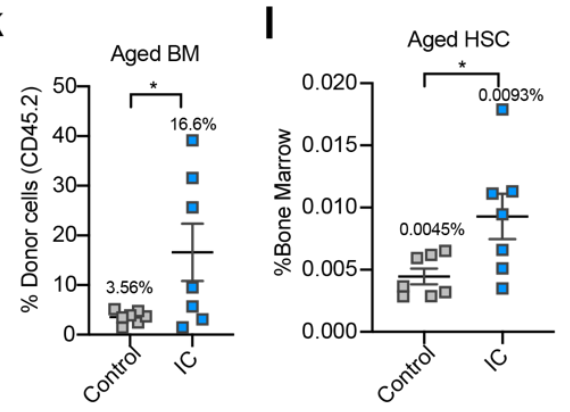

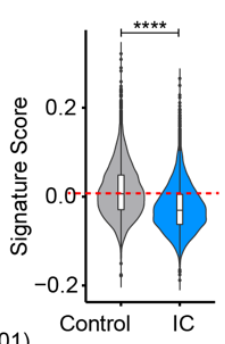
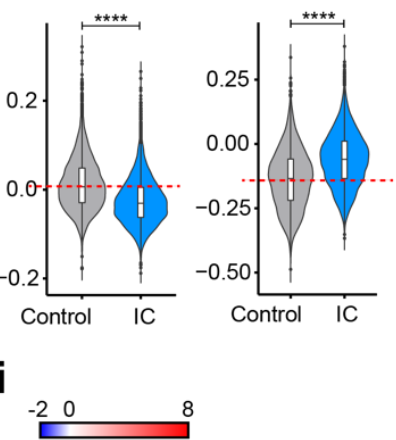

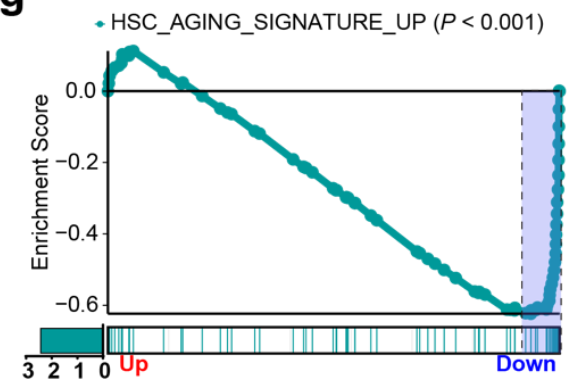

j

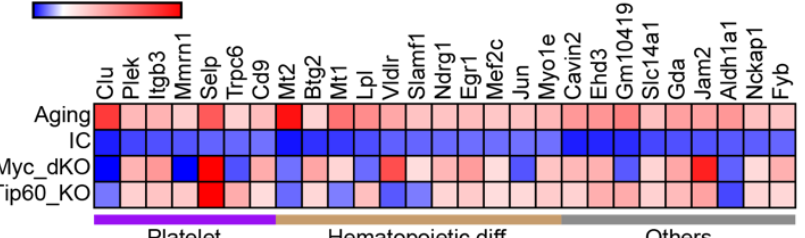

Tip60_KO

m

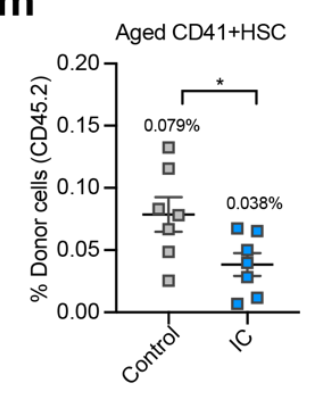

n

0
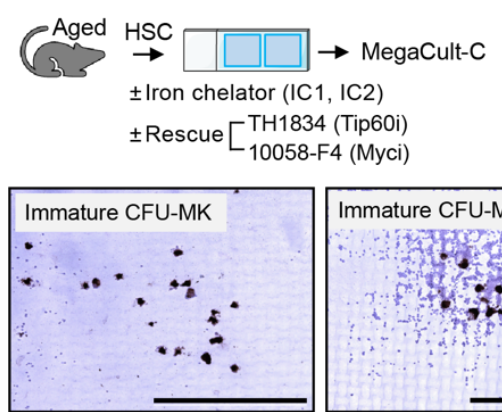
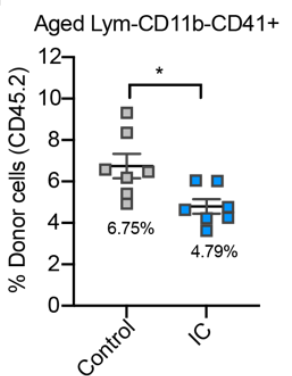

p

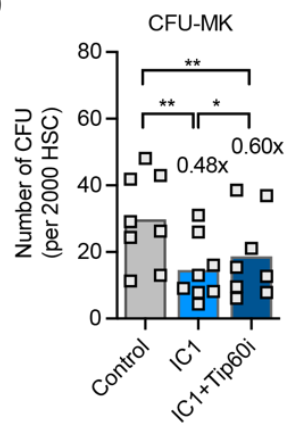
(Control vs. IC, 14d) $\downarrow$ FACS HSC $\downarrow$ $\%$ donor chimerism (16 weeks post BMT)

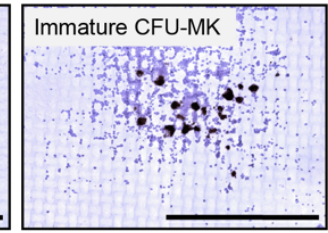

Mature CFU-MK

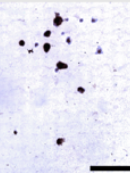


606

607

608

609

610

611

612

613

614

615

616

617

618

619

620

621

622

623

624

625

626

627

628

629

630

631

632

633

634

635

636

637

638

639

640

641

642

643

644

645

646

647

\section{Figure legends}

\section{Figure 1: Activation of the limited iron response increases self-renewal of multipotent HSPC.}

a,b, Quantification of iron responsive elements (IRE) in mRNA transcripts of HSC gene signatures; a, IRE enrichment analysis of HSC gene signatures; b, IRE-motif in Ly6a, Hmga2, and $P b x 1$.

c, IRE enrichment analysis of genes associated with HSC cycling. Cell cycle gene signatures were defined with DEG in Retinoic Acid reporter positive (highly proliferative) compared to negative (less proliferative) mouse HSC (GSE108155).

d-e, Scheme showing experimental design: HPC-7 cells were cultured in the presence of vehicle control (0.01\%DMSO) or Nocodazole for $16 \mathrm{hrs}$ and subjected to sequential Tfrc smRNA FISH followed by phosphoSer10 Histone $\mathrm{H3}(\mathrm{pS} 10 \mathrm{H} 3)$ immunofluorescence to identify mitotic cells (d). e, Left: Representative filtered and overlaid images of treated HPC-7 cells stained by Tfrc smRNA FISH (Cy5, pseudocolor magenta), phosphoSer10 Histone H3 (AlexaFluor 488, pseudocolor yellow) and nuclei (DAPI, pseudocolor gray). Scale bars, $10 \mu \mathrm{m}$; Right: Cells positive for pS10H3 staining are marked as mitotic $(n=80)$ and cells negative for pS10H3 staining are marked as non-mitotic $(n=104)$. Violin plots show absolute number of cytoplasmic Tfrc mRNA molecules per cell.

$\mathbf{f - h}$, Activation of iron homeostasis pathways upon iron chelator (IC1: $5 \mu \mathrm{M}$ DFO; IC2: $3 \mu \mathrm{g} / \mathrm{ml}$ EP) exposure as indicated by rapid degradation of ferritin heavy chain (Fth1) protein in HPC-7 cells (f); Ferritinophagy in HSC mediated by Naco4 activation detected by immunohistochemistry staining (g), $\mathrm{n}=55$ (Control), 66 (IC), 62 (IC+VPS34i) cells; and transferrin uptake in HPC-7 cells $(\mathbf{h}), \mathrm{n}=9 ; 2$ independent experiments.

i, Quantification of phenotypical HSC by scRNA sequencing after 48hrs IC (DFO) exposure (mock-treated HSC as controls). Cells with HIf and Ly6a expression higher than the $25 \%$ percentile of their average expression in all cells in both IC and control groups were defined as HSC (green), otherwise non-HSC (orange).

j-k, Comparison of the expression of IC-treated HSC versus control HSC in scRNA RNA sequencing, with gene signatures associated with HSC proliferation (j, GSE108155) and differentiation (k). Lists of the gene signatures are available in Extended Data Table 1. Signature scores were calculated with Seurat package.

I, Long-term culture-initiating cell (LTC-IC) assay to quantify HSC upon IC exposure alone or along with inhibition of iron homeostasis pathways. Left: Experimental strategy for pharmacological inhibition of iron import and intracellular mobilization. Right: Fold changes of long-term culture-initiating cell (LTC-IC) frequencies compared to controls across individual mice (bar graph). $n=5$. LTC-IC frequencies was estimated by ELDA and shown in parentheses in ELDA plot (right).

If not specified otherwise, data are mean \pm SEM (e, $\mathbf{g}, \mathbf{h}, \mathbf{I}$ (bar graph)). Significance $p$-values, indicated as ${ }^{*} p<0.05,{ }^{* *} p<0.01,{ }^{* * *} p<0.001,{ }^{* * * *} p<0.0001$, n.s. (not significant), were calculated using Student's $t$-test (unpaired: e, g, j, k (bar graph); paired: h), Poisson statistics (I (ELDA plots)). Significance of enrichment was calculated by hypergeometric test $(\mathbf{a}, \mathbf{c})$. 
648

649

650

651

652

653

654

655

656

657

658

659

660

661

662

663

664

665

666

667

668

669

670

671

672

673

674

675

676

677

678

679

680

681

682

683

684

685

686

687

\section{Figure 2: Limited iron response-triggered $\beta$-oxidation of arachidonic acid increases HSPC function.}

a, Pathway network analysis of molecular alterations in HSC upon IC exposure. Left: Following IC (DFO) treatment, HSC were subjected to scRNA-seq and DEG were subjected to pathway analysis by NetworkAnalyst; Right: Significantly enriched pathways $(p<0.05)$ were subjected to EnrichmentMap for network visualization, and clustered with AutoAnnotate based on the coefficient calculated by the overlapping of implicating genes. Each circle represents a pathway, the color intensity is a mapping of enrichment significance $p$-value. Thickness of connecting lines represents the similarity coefficient calculated by EnrichmentMap.

b, Quantification of Hadha levels in different hematopoietic stem and progenitor cell populations 24hrs after IC (DFO) exposure compared to vehicle controls. $n=7$.

c, Assessment of the effect of iron chelation on fatty acid metabolism in Ba/F3 cells, by Seahorse analysis detecting $\beta$-oxidation (after CPT- 1 inhibition with etomoxir) specific oxygen consumption rates (OCR). Endogenous basal (left) and maximal (right) fatty acid oxidation rates are shown. See methods for details in the calculation of $\beta$-oxidation specific OCR.

d, LTC-IC assay to quantify functional HSC upon IC1 (DFO) treatment alone or in combination with pharmacological inhibition of fatty acid import to mitochondria using etomoxir (Etx). Bar graphs represent foldchanges of LTC-IC frequency of chelator treatment compared to control for individual mice. In the ELDA plot, average stem cell frequency is shown for each treatment group. $\mathrm{n}=7$.

e, Quantification of symmetric self-renewal (SS), symmetric differentiation (SD), and asymmetric cell divisions (AD) based on Numb protein (red) abundance. Nucleus was counterstained with DAPI (blue), scale bar indicates $5 \mu \mathrm{m}$. Bar plot shows the quantification of division modes of HSC treated with IC2 (EP) alone or in combination with ferric ammonium citrate (FAC). Shown is the percentage of HSC daughter cell pairs (20-30 cell pairs scored per condition and experiment) for each division mode. $n=2$ independent experiments.

f, Quantification of AA levels by mass spectrometry following IC treatment (IC1: DFO, IC2: EP, IC3: DFX) of HPC-7 cells. $n=3-4$.

g, Quantification of Acsl4 protein levels by Western blot in HPC-7 cells after IC exposure (IC1: DFO, IC2: EP) at the time point of 8,16 and $24 \mathrm{hrs}$.

$\mathbf{h}$, Assessment of fatty acid oxidation rates in BA/F3 cells transduced with non-targeting control (NTC) or shRNA targeting Acs/4 (KD) after IC (DFO) treatment. Basal and maximal fatty acidspecific OCR are shown. $\mathrm{n}=3$.

i, HSC enumeration by LTC-IC following RNAi-mediated ablation of Acs/4 (KD) and IC (DFO) treatment; assessment of shRNA construct vs. a non-targeting control (NTC) shRNA vector. Representative of LTC-IC GFP+ colonies are shown. Bar plot shows the quantification of LTCIC frequency estimated in ELDA plots, $n=3$.

If not specified otherwise, data are mean $\pm \operatorname{SEM}(\mathbf{b}, \mathbf{c}, \mathbf{e}, \mathbf{f}, \mathbf{h}$; $\mathbf{d}$ and $\mathbf{i}$ (bar graph)). Significance $p$-values, indicated as ${ }^{*} p<0.05,{ }^{* *} p<0.01$, n.s. (not significant), were calculated using paired Student's t-test (b, c, e, f, and $\mathbf{h}$ ), or Poisson statistics (d, i (ELDA plots)). 
689

690

691

692

693

694

695

696

697

698

699

700

701

702

703

704

705

706

707

708

709

710

711

712

713

714

715

716

717

718

719

720

721

722

723

724

725

726

727

728

729

730

\section{Figure 3: Tip60 controls HSC-typic gene expression upon activation of the limited iron response.}

a, Quantification of HSC frequency by LTC-IC assay after IC treatment alone or in combination with ATP citrate lyase inhibition (ACLYi). $n=4$. Bar graphs represent foldchanges of LTC-IC frequency of chelator treatment compared to control for each individual mouse. Average LTC-IC frequencies are shown in parentheses. Significance tested using ELDA, ${ }^{*} p<0.05$.

b, GSEAPreranked enrichment analysis of scRNA-seq data using gene set of Tip60 binding targets in ESC, or DEG in Tip60-KO LSK. Bar plot shows the normalized enrichment score (NES, absolute value) in the respective gene set.

c, Balloon plot showing GSEAPreranked enrichment analysis performed with gene sets associated with histone modifications and Tip60 binding. NES and significance $p$-values of gene sets with all the genes associated with each of the histone modifications and those common with Tip60 binding targets are shown.

d, Upstream regulator analysis of differentially expressed genes upon IC exposure of HSC that are also direct targets of Tip60.

e, Expression changes of Tip60/Myc target genes in HSC after IC treatment alone or in combination with inhibition of c-Myc (Myci) or Tip60 (Tip60i) by Fluidigm analysis. Fold changes of genes across treatment groups from Fluidigm and scRNA-seq (SC) are shown. Significance of differential expression shown (right panel) were estimated by paired Student's $t$-test comparing the $\Delta^{\mathrm{Ct}}$ in IC versus control, or co-treatment versus IC. ${ }^{*} p<0.05,{ }^{* *} p<0.01,{ }^{* * *} p<$ $0.001, n$, not significant.

f-g, Quantification of HSC by LTC-IC assay after IC treatment alone or in combination with Tip60, or Myc inhibition. $n=4$. Average LTC-IC frequencies are shown in parentheses. Significance tested using ELDA, * $p<0.05$.

\section{Figure 4: Ageing associated cytoplasmic iron loading impairs HSPC function.}

a,b, Increased labile iron pool (LIP) in aged HSC compared to young HSC. Cytoplasmic Fe ${ }^{2+}(\mathbf{a})$ or $\mathrm{Fe}^{3+}$ (b) LIP were measured using FeRhoNox-1 or Calcein-AM staining, respectively. For Calcein-AM assay, EP was used for the dequenching of Calcein-bound iron. Quantification of FeRhoNox-1 and Calcein-AM mean fluorescence intensity (MFI in arbitrary units (a.u.)) by FACS analysis are shown. $n=4-6$.

c, Comparison of IPA upstream regulator analysis of DEG in Fbxl5-KO HSC (GSE93649), previously defined mouse HSC ageing signatures (GSE166674), and ageing-associated proteins in human plasma (PMID33089916). Z-scores of top 30 upstream regulators are shown.

d,e, Quantification of IRE-containing transcripts of murine HSC ageing signatures (d), or DEG of human aged (65 to 75 year old) compared to young (18 to 30 year old) HSC (e, GSE10440). Significance of enrichment was calculated by hypergeometric test.

f, Comparison of the expression of IC-treated HSC versus control HSC in scRNA sequencing, with up- or down-regulated genes in murine aged HSC signatures. Signature scores were calculated with Seurat package.

g, GSEAPreranked analyses of IC-treated HSC scRNA-seq data using gene sets of upregulated signatures in murine aged $\mathrm{HSC}(\mathbf{g})$. 
731 h, GSEAPreranked analyses of IC-treated HSC scRNA-seq data using gene sets of 'MEGAKARYOCYTE_DIFFERENTIATION' retrieved from the gene ontology (GO) database, as well as significantly up-regulated genes in aged versus young HSC that are positive for von Willebrand Factor ( $\mathrm{VWF}+$ ). Single cell RNA sequencing data of aged and young HSC were obtained from GSE70657; HSC with vWF expression > 3 were defined as vWF+.

736 i, Expression of genes in leading edge of GSEA in $(\mathbf{g})$ was compared in aged HSC, IC-treated

737 HSC, as well as Tip60 KO LSK or Myc dKO HSC. Genes implicated in platelet activation or

738 production, as well as hematopoietic differentiation are indicated.

739 j, Scheme of transplantation assay to evaluate the effect of iron chelation on aged HSC. HSC

740 were isolated from aged mice following 2-week treatment of iron chelator (DFO) or vehicle

741 control (PBS), and transplanted into congenic recipients (500 HSC each) via retro-orbital

742 injection. Donor cell chimerism was assessed by FACS analysis 16 weeks after transplantation.

$743 \mathbf{k}$,l, Donor cell $\left(\right.$ CDD45.2+ $2^{+}$chimerism (k) and frequency of donor-derived HSC (I) in recipient 744 bone marrow 16 weeks after transplantation.

745

$\mathbf{m}, \mathbf{n}$, Frequency of CD41+ HSC $(\mathbf{m})$, and megakaryocyte-primed progenitors (CD3-/CD4-/CD8-/ B220-/CD11b-/CD41+; $\mathbf{n}$ ) within the donor-derived cells.

747

o,p, Quantification of megakaryocytic differentiation of aged HSC upon IC (IC1: DFO) exposure alone or in combination with Tip60 or Myc inhibition for 7 days. Representative morphologies of megakaryocytic colony-forming unit (CFU-Mk) are shown (o). Quantification of CFU-Mk colonies across different conditions were normalized to 2000 HSC plated (p). $n=8$; 3 independent 751 experiments.

752 If not specified otherwise, data are mean $\pm \operatorname{SEM}(\mathbf{a}, \mathbf{b}, \mathbf{k}-\mathbf{n}$, and $\mathbf{p})$. Significance $\mathbf{p}$-values, 753 indicated as ${ }^{*} p<0.05,{ }^{* *} p<0.01,{ }^{* * *} p<0.001,{ }^{* * *} p<0.0001$, were calculated using Student's 754 t-test (unpaired: $\mathbf{a}, \mathbf{b}, \mathbf{f}$, and $\mathbf{k}-\mathbf{n}$; paired: $\mathbf{p}$ ). 
755

756

757

758

759

760

761

762

763

764

765

766

767

768

769

770

771

772

773

774

775

776

777

\section{Methods}

\section{Chemicals and Reagents}

Eltrombopag (EP, pure compound provided by Novartis) was reconstituted in sterile distilled water as $1 \mathrm{mg} / \mathrm{ml}$ stock and was stored at ambient temperature, light-protected for up to 2 weeks. Deferoxamine (DFO, Sigma) was freshly prepared with sterile distilled water for every experiment. Anti-CD71 blocking antibody (MCA2396EL, Bio-Rad) and Etomoxir (Sigma) were stored at $-20^{\circ} \mathrm{C}$ in aliquots of $1 \mathrm{mg} / \mathrm{ml}$ and $10 \mathrm{mM}$ stocks respectively. Inhibitors for VPS34 (RGNCY-0041/0042, Reagency), Myc (10058-F4, Selleck) and Tip60 (TH1834, Axon) were reconstituted in dimethyl sulfoxide (DMSO) and stored at $-80^{\circ} \mathrm{C}$ until use. Iron chemo-sensors Calcein-AM (Invitrogen) and FeRhoNox-1 (Goryo Chemical) were stored light-protected at $-20^{\circ} \mathrm{C}$ and were freshly reconstituted in DMSO for every experiment. ATP citrate lyase (ACLY) inhibitor SB 204990 (Tocris, Minneapolis, MN) and Senexin A (Selleck Chemicals, Houston, TX) were reconstituted in DMSO with a stock concentration of $10 \mathrm{mM}$ and stored at $-20^{\circ} \mathrm{C}$ until use. Cortistatin $\mathrm{A}$ was obtained from the laboratory of Matthew Shair at Harvard University, and reconstituted in DMSO with a stock concentration of $1 \mathrm{mM}$ and stored at $-20^{\circ} \mathrm{C}$ until use.

\section{Mice and cell lines}

C57/BL6 (stock number: 000664), Pepc/BoyJ mice (002014), and Fth1 ${ }^{\text {LoxP }}$ mice (018063) were purchased from Jackson Laboratories and housed in animal facilities at the Albert Einstein College of Medicine. Male and female mice at the age of 6-10 weeks were utilized for the experiments. All experiments were approved by the Institutional Animal Care and Use Committee of the Albert Einstein College of Medicine (Protocol\# 0000-1015). All procedures were performed in accordance with guidelines from the Institutional Animal 
778 Care and Use Committee of the Albert Einstein College of Medicine. The murine 779 multipotent progenitor cell line HPC-7 was provided by Dr. Omar Abdel-Wahab (Memorial Sloan Kettering Cancer Center). Murine BA/F3 and human 293T cell lines were purchased from ATCC. All cell lines were routinely monitored for and testing negative for mycoplasma.

Cell culture

Cell lines. HPC-7 cells were passaged in IMDM with 5\% fetal bovine serum (FBS), $1 \%$ penicillin/streptomycin, $1 \%$ sodium pyruvate, $6.9 \mathrm{ng} / \mathrm{mL}$ monothioglycerol (Sigma) and $100 \mathrm{ng} / \mathrm{ml}$ recombinant mouse $(\mathrm{rm}) \mathrm{SCF}^{111}$. BA/F3 cells were cultured in RPMI 1640 with 10\% FBS, 2 ng/ml rmIL-3 and 1\% penicillin/streptomycin ${ }^{112}$. 293T cells were cultured in isolated by cell sorting on a Moflo Astrios EQ (Beckman Coulter). Lin ${ }^{-}$Sca- $1^{+} \mathrm{C}-$ maintained at $37^{\circ} \mathrm{C}$ and $5 \% \mathrm{CO}_{2}$ unless otherwise specified.

\section{Flow cytometry analysis and sorting}

Cell preparation and sorting. Isolation of mononuclear cells (MNC) from mouse bone 
801 conjugated, rotating at $4^{\circ} \mathrm{C}$ for $30 \mathrm{~min}$. Cells were washed and then stained with triplewashed anti-IgG magnetic beads (Untouched Mouse T Cells Kit, Thermo Fisher) rotating at $4^{\circ} \mathrm{C}$ for $30 \mathrm{~min}$. Cells were washed and then depleted of lineage-positive cells by passing through a magnetic separation column (MACS LD Column, Miltenyi Biotec) loaded on a DynaMag-5 Magnet (Invitrogen). Lineage-negative cells were then stained

806 for 30 min on ice with stem and progenitor cell markers (Sca-1, c-Kit, CD150, CD48, 807 CD34; 1:100). A detailed description of mouse antibodies can be found in Extended Data Table 10. $\mathrm{Lin}^{-} \mathrm{Sca}-1^{+} \mathrm{C}-\mathrm{Kit}^{+} \mathrm{CD} 150^{+} \mathrm{CD}_{4} 8^{-}$(HSC) or $\mathrm{Lin}^{-} \mathrm{Sca}-1^{+} \mathrm{C}-\mathrm{Kit}^{+} \mathrm{CD} 150^{+} \mathrm{CD} 48^{-} \mathrm{CD}^{-} 4^{-}$

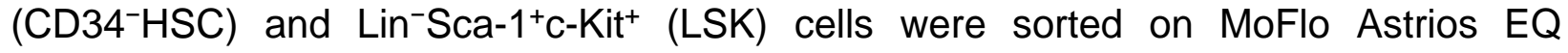
(Beckman Coulter). Quantification of CD71. Lin- cells were stained for cell surface markers defining stem and progenitor cells (HSPC), as well as cell surface transferrin receptor (CD71, eBioscience). Stained cells were then fixed with Cytofix/Cytoperm buffer 813 (BD Bioscience, 554722) for 20 min on ice, washed twice with Perm/Wash buffer (BD 814 Bioscience, 554723), re-suspended in Perm/Wash buffer containing anti-CD71 antibody 815 (abcam, ab84036) and incubated for 1 hour at room temperature (RT). After 2 washes with Perm/Wash buffer, cells were re-suspended in Perm/Wash buffer containing Alexa

817 Fluor 647 goat anti-rabbit IgG $(\mathrm{H}+\mathrm{L})$ (Invitrogen, A21245) for 30 min at RT. Cells were 818 washed twice with Perm/Wash buffer and subjected to FACS analysis. Quantification of 819 Hadha. For primary cells, lineage-depleted MNC were treated with iron chelators (10 $\mu \mathrm{M}$ 820 DFO) for 24 hours, followed by staining with FACS antibodies against surface markers 821 for HSPC. Thereafter, stained cells were fixed and permeabilized with Cytofix/Cytoperm 822 buffer for 20 min on ice. For HPC-7, cells were treated with iron chelators (10 $\mu$ M DFO or $8235 \mu \mathrm{g} / \mathrm{ml} \mathrm{EP)} \mathrm{for} 24$ hours before fixation and permeabilization with Cytofix/Cytoperm 
824 buffer. Intracellular staining with anti-Hadha antibody (abcam, ab203114) for 1 hour at 825 RT, followed by secondary staining with Alexa Fluor 647 goat anti-rabbit $\lg G(\mathrm{H}+\mathrm{L})$

826 (Invitrogen, A21245) for $30 \mathrm{~min}$ at RT. Calcein-AM. Intracellular iron levels were

827 measured by calcein-acetoxymethyl ester (calcein-AM, Invitrogen) assay as previously

828 described ${ }^{44,113}$. Primary bone marrow MNC or HPC7 cells were preloaded with 50 nM

829 calcein-AM (Invitrogen) at $37^{\circ} \mathrm{C}$ for $5 \mathrm{~min}$, before the treatment with vehicle control or iron

830 chelators for 2 to 6 hours at $37^{\circ} \mathrm{C}$. Treatments were performed in complete media

831 (Extended Data Fig. 3h, 4 to 6 hr exposure) or PBS supplemented with 1\% polyvinyl alcohol (Extended Data Fig. 8b, 2 hr exposure) to model the ability of cells to access transferrin-bound iron extracellularly. For quantifying $\mathrm{Fe}^{3+}$ cytoplasmic pool, a ferric ironspecific chelator (eltrombopag, Novartis) was used to liberate calcein-bound $\mathrm{Fe}^{3+}$ iron (Extended Data Fig. 8b). For primary MNC, cells were stained with antibodies against

836 cell surface markers for HSPC on ice for 15 min. Thereafter, intracellular calcein fluorescence was analysed by flow cytometry. The relative size of the labile iron pool (LIP) was calculated as the difference in mean fluorescence intensity $(\Delta M F I)$ between the vehicle and iron chelator-treated cells ${ }^{114}$. FeRhoNox-1. Intracellular iron levels were also

840 measured with FeRhoNox-1 fluorescent imaging probe specific for ferrous iron (Goryo 841 Chemical). For primary cells, lineage-depleted MNC were incubated with $20 \mu \mathrm{M}$

842 FeRhoNox-1 at $37^{\circ} \mathrm{C}$ for 1 hour. Antibody cocktail for HSPC surface staining was added 843 in the last 10 min of FeRhoNox-1 incubation. For HPC-7, cells were pre-treated with 844 VPS34 inhibitor (Reagency, RGNCY-0042) for 2 hours before exposure to vehicle or iron 845 chelator $\left(10 \mu \mathrm{g} / \mathrm{ml}\right.$ eltrombopag) for 1 hour at $37^{\circ} \mathrm{C}$. Thereafter, treated cells were washed 
847 twice with PBS and subjected to FACS analysis. Unless otherwise specified, fluorescent signals were acquired with BD FACS Aria II system (Becton Dickinson) and analysed with FlowJo V10.2.

\section{Single molecule RNA FISH}

851 To design Tfrc mRNA-specific probes for sequential single molecule FISH (smFISH), full 852 length transcript of Tfrc (NM_011638) was used as input for PaintSHOP ${ }^{115}$ to retrieve 22

853 primary targeting sequences (30-40bp, Extended Data Table 11), separated by at least

854 10bp. Putative sequences were then screened for off-target activity using NCBI Blast 855 (https://blast.ncbi.nlm.nih.gov/Blast.cgi) against mouse transcriptome. Selected 856 sequences were then concatenated on the $5^{\prime}$ and $3^{\prime}$ end with flanking readout 20 mer 857 sequences (GTTTGAAGATTCGACCTGGA), generating a final 'primary probe’. SmFISH immunofluorescence staining procedure and analysis were performed as described previously ${ }^{58,116}$. Briefly, treated and control cells were attached to coverslips using biotinylated anti-CD44 coating ${ }^{117}$. Residual media was washed with PBS, and cells were fixed in 3.2\% PFA (Electron Microscopy Sciences), diluted in $\mathrm{PBS}$ with $1 \mathrm{mM} \mathrm{MgCl}_{2}$

862 (PBSM), for 10 minutes at room temperature. Cells were then permeabilized in $0.1 \%$

863 Triton X-100 in PBSM for 10 minutes. After washing with PBSM, cells were incubated at 864 room temperature with $30 \%$ prehybridization buffer (30\% formamide, 2 X saline-sodium 865 citrate buffer) for 30 minutes. Primary hybridization was done in $30 \%$ hybridization buffer 866 consisting of $10 \%$ dextran sulfate, $30 \%$ formamide, 2 X saline-sodium citrate (SSC), $2 \mathrm{mM}$

867 VRC, $10 \mu \mathrm{g} / \mathrm{ml}$ sheared ssDNA from salmon sperm, $10 \mu \mathrm{g} / \mathrm{ml}$ E. coli tRNA, $10 \mu \mathrm{g} / \mathrm{ml}$ 868 molecular grade bovine serum albumin (BSA), and $200 \mathrm{ng}$ of primary probe mixes, 869 overnight at $37^{\circ} \mathrm{C}$. Thereafter, cells were washed twice with $30 \%$ pre-hybridization buffer 
870 for $20 \mathrm{~min}$ at $37^{\circ} \mathrm{C}$ and once with $2 \mathrm{X}$ SSC. Cells were then post fixed in $3.2 \%$ PFA in

871 PBSM for 10 min, followed by washing in 2X SSC. Primary stained cells were incubated

872 with $10 \%$ prehybridization buffer (10\% formamide, $2 \mathrm{X} \mathrm{SSC}$ ) for $10 \mathrm{~min}$ at $37{ }^{\circ} \mathrm{C}$ and

873 stained with $10 \%$ dextran sulfate, $10 \%$ formamide, 2 X SSC, $2 \mathrm{mM} \mathrm{VRC,} 10 \mu \mathrm{g} / \mathrm{ml}$ sheared

874 ssDNA from salmon sperm, $10 \mu \mathrm{g} / \mathrm{ml}$ E. coli tRNA, $10 \mu \mathrm{g} / \mathrm{ml}$ molecular BSA, and $10 \mathrm{ng}$

875 Cy5-labelled readout probe of 20mer readout probe (RO2-Cy5) for Tfrc gene for $3 \mathrm{hrs}$ at

$87637^{\circ} \mathrm{C}$. Cells were then washed twice for $10 \mathrm{~min}$ in $10 \%$ prehybridization buffer, followed

877 by a final wash in 2X SSC. Before the immunostaining for pS10H3, cells were again fixed with 3.2\% PFA in PBS for 10 min at room temperature (RT). Cells were washed once with PBS for 5 min at room temperature. Blocking was performed in blocking buffer (PBS, 1\% 880 RNAse-free BSA, $0.2 \%$ Triton X-100) for 30 min at room temperature. Next, cells were 881 incubated with the primary antibody (1:200 mouse anti-pS10H3, Cell Signaling 882 Technology) in antibody dilution buffer (PBS, 0.1\% BSA, 0.1\% Triton X-100) overnight at 883 $4{ }^{\circ} \mathrm{C}$. Next day, cells were washed thrice with PBS for 5 min at RT before incubation with the secondary antibody (1:200 rabbit anti-mouse AlexaFluor 488, Cell Signaling 885 Technology) in antibody dilution buffer for 1 hour at RT. Excess antibody was removed 886 by washing cells with $1 \times \mathrm{PBS}$ for $5 \mathrm{~min}$ at RT. Cells were then mounted in Prolong

887 Diamond Antifade reagent plus DAPI (Invitrogen). Images were acquired using oil 888 immersion 100X objective on an epifluorescence Olympus Digital Station 6 microscope.

889 Exposure times were 1000 ms, 50 ms, 100 ms for Cy5, AlexaFluor 488 and DAPI 890 respectively. Z stacks spanning the entire volume of the cells were acquired by imaging 891 every $300 \mathrm{~nm}$ along the z-axis. Acquisition control of the microscope was achieved using 892 IPLab software. For data analysis, single molecule mRNA and transcription site detection 
893 was performed using freely available and MATLAB-written software FISHquant, by 3D

894 Gaussian fitting of thresholded spots, implemented in MATLAB R2020b ${ }^{117}$. Further

895 experimental details, validation, and discussion of this methodology in the hematopoietic

896 system can be found in ${ }^{58}$.

\section{Stroma-free long-term culture-initiating cell assay}

898 Conditioned media derived from mouse stromal cells were collected as previously described $^{44}$. Long-term culture-initiating cell (LTC-IC) assays were performed as

900 previously described ${ }^{44}$ for the assessment of the frequency of functional stem cells ex

901 vivo. Briefly, limiting dilutions of LSK cells were FACS-sorted into 96-well plates

902 containing Myelocult M5300 and conditioned media at 1:1 ratio, supplemented with 50

$903 \mathrm{ng} / \mathrm{ml} \mathrm{rmSCF}, 50 \mathrm{ng} / \mathrm{ml} \mathrm{rmTPO}, 1 \mu \mathrm{M}$ hydrocortisone (STEMCELL Technologies), and

$904200 \mu \mathrm{g} / \mathrm{ml}$ Primocin. After 4 weeks of culture at $32^{\circ} \mathrm{C}, 5 \% \mathrm{CO}$, limiting dilutions of LSK

905 cells and their respective replicate wells were subjected to methylcellulose colony assay

906 with $\mathrm{HSC} 007$ (R\&D Systems) at $37^{\circ} \mathrm{C}, 5 \% \mathrm{CO}_{2}$ for 1 week. Colony forming units were

907 identified and scored using inverted light microscope. Stem cell frequency was estimated

908 using extreme limiting dilution analysis (ELDA) algorithm ${ }^{118}$.

909 For inhibiting the activation of iron homeostasis regulatory pathways upon intracellular

910 iron reduction, a CD71-blocking antibody (Bio-Rad, MCA2396EL) ${ }^{119}$ and VPS34 inhibitor

911 (Reagency, RGNCY-0041) ${ }^{120}$ were used to simultaneously block iron uptake and

912 mobilization, respectively. For inhibiting fatty acid oxidation, etomoxir (Sigma, E1905) was

913 used to irreversibly block mitochondrial carnitine palmitoyltransferase-121,122. For

914 inhibiting Myc, 10058-F4 ${ }^{123}$ (Selleck, S7153) was used to block the dimerization of Myc 
915 and Max. For inhibiting histone acetyltransferase activity of Tip60, TH1834 (Axon, 2339) ${ }^{124}$ was used.

917 Colony-forming unit assays of megakaryocyte progenitors

918 For the detection of colony-forming unit (CFU) of mouse megakaryocyte progenitors, 9192000 sorted HSC ( $\left.\mathrm{Lin}^{-} / \mathrm{Sca} 1^{+} / \mathrm{CKit}^{+} / \mathrm{CD} 150^{+} / \mathrm{CD} 48^{-}\right)$were plated in a collagen-based 920 medium in double chamber culture slides and cultured for 7 days at $37^{\circ} \mathrm{C}, 5 \% \mathrm{CO}_{2}$

921 (MegaCult®-C Medium without Cytokines, STEMCELL Technologies). Cultures were 922 supplemented with human recombinant $50 \mathrm{ng} / \mathrm{ml}$ TPO (Peprotech), $20 \mathrm{ng} / \mathrm{ml}$ human 923 recombinant IL-6 (STEMCELL Technologies) and $10 \mathrm{ng} / \mathrm{ml}$ mouse recombinant IL-3 924 (R\&D) according to manufacturer's instructions. Staining for acetylcholinesterase content 925 and scoring of CFU-Mk colonies were performed according to manufacturer's protocol, 926 and colonies with at least eight-cell cluster were scored using Inverted Infinity and Phase 927 Contrast Microscope (Fisher Scientific). Where indicated, media were supplemented with: $92810 \mu \mathrm{M}$ DFO (Sigma), $3 \mu \mathrm{g} / \mathrm{ml}$ eltrombopag (Novartis), $10 \mu \mathrm{M}$ Tip60 histone 929 acetyltransferase inhibitor (TH1834, Axon), 25 uM c-Myc inhibitor (10058-F4, Selleck 930 Chemicals).

\section{Bone marrow transplantation}

932 Aged mice (22-24 mos) obtained from National Institutes for Aging were subjected to in 933 vivo treatment of iron chelator (Deferoxamine, $50 \mathrm{mg} / \mathrm{kg}$ ) or vehicle control (sterile PBS) 934 daily for 14 days. Treatments were administered by intraperitoneal injection. Thereafter, $935 \mathrm{HSC}\left(\mathrm{Lin}^{-} / \mathrm{Sca} 1^{+} / \mathrm{CKit}^{+} / \mathrm{CD} 150^{+} / \mathrm{CD} 48-\right)$ were prospectively FACS-sorted from treated mice 936 and pooled according to experimental group, where $500 \mathrm{HSC}$ along with $1 \times 10^{6}$ Sca-1937 depleted CD45.2 Pepc/BoyJ BMMNC were transplanted into CD45.2 recipient mouse 
938 irradiated with 2 rounds of 500 rads irradiation. Detailed procedures for HSC

939

940

941

942

943

944

945

946

947

948

949

950

951

952

953

954

955

956

957

958

959

transplantation were described previously ${ }^{44}$. Chimerism of donor-derived hematopoietic

stem and progenitor, as well as lineage reconstitution were assessed at 16-weeks post

transplantation and analyzed with BD FACS Aria II system (Becton Dickinson) and FlowJo V10.2. Detailed information of the antibodies used for donor chimerism analysis can be found in Extended Data Table 10.

\section{RNA extraction, quantitative PCR and microarray}

RNA extraction, reverse transcription, and quantitative PCR (qPCR) were performed as previously described ${ }^{44}$. Briefly, RNA was extracted with the Qiagen RNeasy Micro Kit, and reverse-transcription of extracted RNA was performed using Superscript II reverse transcriptase (Invitrogen). For qPCR, $10 \mu$ reaction volume containing $2 \mu \mathrm{l}$ cDNA (5 $\mathrm{ng} / \mu \mathrm{l}), 0.5 \mu \mathrm{l}$ of each forward and reverse target primers $(5 \mu \mathrm{M}), 5 \mu \mathrm{l}$ of Power SYBR Green mix (Applied Biosystems), and $2 \mu$ of nuclease-free water were used. Triplicate samples and five serial dilutions of standards were prepared for each target gene. Thereafter, qPCR was performed using ViiA 7 Real-Time PCR system (Applied Biosystems) according to the manufacturer's instructions. Gene expression levels were calculated based on the standard curve with subsequent normalization to internal control $(G A P D H)$. A list of qPCR primers for target genes can be found in Extended Data Table 11.

For microarray, 5 to 20 ng of RNA were amplified and labelled with the GeneChip 3' IVT Pico Kit (Affymetrix) according to manufacturer's protocols. Labelled RNA was then hybridized to Mouse Clariom S microarrays (Affymetrix), scanned and analysed with 
960

961

962

963

964

965

966

967

968

969

970

971

972

973

974

975

976

977

978

979

980

981

982

GeneChip Scanner 3000 7G system (Affymetrix) according to standard protocols. Resulting CEL files have been deposited in the GEO database (GSE157817).

\section{Analysis of microarray data}

Microarray data was analysed as previously described ${ }^{44}$. Briefly, quality assessment of microarray results was performed with Transcriptome Analysis Console 3.0 (Affymetrix). After data normalization with RMA algorithm of Oligo Bioconductor package ${ }^{125}$, paired, linear modelling of limma package was used for differential expression analysis ${ }^{126}$. Microarray expression probes were then annotated to gene names with annotation files obtained from the Affymetrix website (http://www.affymetrix.com).

Differentially expressed genes (DEG; fold change $>1.6$ and $P<0.05$ ) were subjected to Ingenuity Pathway Analysis (IPA, Qiagen) for the analysis of biological function and upstream regulators. DEG were also examined for the presence of Iron Response Element (IRE) using SIREs Web server 2.0 as previously described ${ }^{127-129}$. Significance of enrichment of IRE-containing genes was calculated by hypergeometric test with built-in phyper function of R (https://www.R-project.org).

\section{Single cell RNA sequencing (scRNA-seq)}

FACS-sorted HSC ( $\left.\mathrm{Lin}^{-} \mathrm{Sca}-1^{+} \mathrm{C}-\mathrm{Kit}^{+} \mathrm{CD} 150^{+} \mathrm{CD} 48^{-} \mathrm{CD} 34^{-}\right)$were exposed to iron chelator $\left(10 \mu M\right.$ DFO) or vehicle $\left(\mathrm{H}_{2} \mathrm{O}\right)$ for 48 hours. Treated cells were collected; viability of $>95 \%$ was confirmed by trypan blue exclusion. 20,000 cells from each treatment group were subjected to $10 x$ Genomics platform for library preparation using Chromium Single Cell 3' Reagent Kits (v3) following the manufacturer's sample preparation guide (PN CG00054 Rev B; Fluidigm). Following quality control assessment with Agilent 2100 Bioanalyzer, approximately $50 \mathrm{ng}$ libraries were sequenced on the BGISEQ-500 platform with 
28+8+91bp reads (read 1: 28bp; read 2: $91 \mathrm{bp}$ plus $8 \mathrm{bp}$ for index) using two lanes per sample. Sequencing data were deposited on GEO database (GSE157821).

For bioinformatic analysis, cell barcode processing, transcriptome alignment (mm10), and gene UMI counting were performed for each of the samples using the count module of Cell Ranger v3.0.2 $2^{129}$. Samples were aggregated using the aggr module with default parameters. Cells with UMI counts $<2,000$ or $>10,000$ were excluded from further analysis to rule out contamination from potentially dead or non-single cells. Cells with mitochondrial mRNAs $>15 \%$ were also excluded. Moreover, genes that were expressed in less than 50 cells were excluded from the expression matrix for downstream analysis. The expression data was then normalized by the total number of unique reads, multiplied by a scale factor of 10,000 and log-transformed. For phenotypic analysis, HSC were defined based on the expression of HSC markers HIf and $L y 6 a^{130,131}$. Cells with expression of $H I f$ and $L y 6 a$ higher than the $25 \%$ percentile of their average expression in all cells in both conditions (vehicle vs. iron chelator) were defined as HSC (green), otherwise non-HSC (orange). Frequencies of phenotypic HSC and non-HSC were visualized separately using Loupe browser v3.1.1 with t-SNE maps. Signature score in each single HSC was calculated with AddModuleScore module of Seurat package.

\section{Gene expression analysis by Fluidigm}

Sorted HSC $\left(\mathrm{Lin}^{-} \mathrm{Sca}-{ }^{+}{ }^{+} \mathrm{C}-\mathrm{Kit}^{+} \mathrm{CD} 150^{+} \mathrm{CD} 48^{-}\right)$were treated with vehicle control, iron chelator (IC, $10 \mu \mathrm{M}$ DFO), as well as IC along with inhibition of c-Myc (Myci, $50 \mu \mathrm{M} 10058$ F4) or Tip60 (Tip60i, $20 \mu \mathrm{M}$ TH1834). Forty-eight hours post treatment, RNA extraction and reverse transcription were performed as described above. Taqman assays (Applied Biosystems) of target genes for Fluidigm gene expression analysis are listed in Extended 
1006 Data Table 11. For pre-amplification of cDNA, reaction mixtures containing $1 \mu \mathrm{L}$ PreAmp 1007 Master (Fluidigm, CA), $1.25 \mu \mathrm{L}$ pooled 0.2x TaqMan assays, and $2.75 \mu \mathrm{L}$ cDNA product were used. PCR amplification was performed as follows: $95^{\circ} \mathrm{C}$ for 2 min; 14 cycles of $95^{\circ} \mathrm{C}$ for $15 \mathrm{sec}$ and $60^{\circ} \mathrm{C}$ for $4 \mathrm{~min}$. No template control (NTC) was included in the preamplification as negative control. For sample Pre-Mix solution preparation, $2.25 \mu \mathrm{L}$ pre1011 amplification product was mixed with $2.5 \mu \mathrm{L}$ TaqMan Fast Advanced Master Mix (Applied 1012 Biosystems) and 0.25 $\mu \mathrm{L}$ 20X GE sample loading reagent (Fluidigm). Thereafter, sample 1013 Pre-Mix solutions were transferred to a 96-well plate. For 10X TaqMan assay preparation, $10145 \mu \mathrm{L}$ of each $20 \mathrm{X}$ TaqMan assay was diluted with $5 \mu \mathrm{L} 2 \mathrm{X}$ assay loading reagent 1015 (Fluidigm), followed by transferring to a 96-well plate. Thereafter, plates containing 1016 sample Pre-Mix solutions and TaqMan assays were loaded onto 96.96 Dynamic Array 1017 IFC (Fluidigm) in Biomark HD system (Fluidigm). Each of the sample Pre-Mix solutions 1018 was mixed with each TaqMan assay in the IFC by Biomark system, and qPCR reactions 1019 were performed according to manufacturer's protocol.

1020 Data were analysed with Fluidigm Real-Time PCR Analysis Software v4.5.1 to obtain the 1021 Ct values of genes in each sample. For differential expression analysis, Delta $\mathrm{Ct}(\Delta \mathrm{Ct})$ of 1022 each gene was calculated by comparing the Ct value to the internal control Actb. Delta 1023 delta $\mathrm{Ct}(\Delta \Delta \mathrm{Ct})$ and fold change $\left(2^{-\Delta \Delta \mathrm{Ct}}\right)$ were calculated by comparing $\Delta \mathrm{Ct}$ in each 1024 treatment condition to $\Delta \mathrm{Ct}$ in control.

1025 Immunofluorescence staining

1026 The following primary antibodies were used for immunofluorescence: anti-Ncoa4 (Santa 1027 Cruz Biotechnology, sc-15984) ${ }^{59}$, anti-Numb (abcam, ab4147) ${ }^{132}$ and anti-CD71 (abcam, 1028 ab84036) ${ }^{133}$. The secondary antibodies used were: Alexa Fluor 594 donkey anti-goat lgG 
$1029(\mathrm{H}+\mathrm{L})$ (Invitrogen, A-11037) and Alexa Fluor 488 goat anti-rabbit IgG $(\mathrm{H}+\mathrm{L})$ (Invitrogen, 1030 A-11008).

1031 Cell culture and pre-treatment: HSC ( $\left.\mathrm{Lin}^{-} \mathrm{Sca}-1^{+} \mathrm{C}-\mathrm{Kit}^{+} \mathrm{CD} 150^{+} \mathrm{CD} 48^{-}\right)$from wildtype

1032

1033

1034

1035

1036

1037

1038

1039

1040

1041

1042 treatment.

1043

1044

1045

1046

1047

1048

1049

1050

1051

C57BL/6 mice were sorted into 16-well chamber slides (Thermo Fisher Scientific) coated with RetroNectin (Takara Bio). HSC were cultured in M5300 media with $100 \mu \mathrm{g} / \mathrm{ml}$ Primocin supplemented with $100 \mathrm{ng} / \mathrm{ml} \mathrm{rmSCF}$ and $50 \mathrm{ng} / \mathrm{ml} \mathrm{rmTPO}$. Ncoa4-mediated ferritinophagy assessment: HSC were subjected to either vehicle $\left(\mathrm{H}_{2} \mathrm{O}\right)$ or iron chelator (DFO, Sigma) treatment for 48 hours. For rescue, VPS34 inhibitor (Reagency, RGNCY0041) was added along with iron chelator. To allow the detection of Ncoa4 foci, chloroquine (10 $\mu \mathrm{M}$, Sigma) was added in the last 4 hour of culture to impair degradation

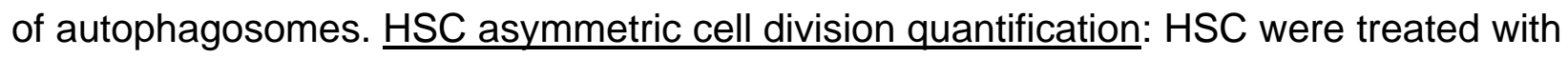
either vehicle $\left(\mathrm{H}_{2} \mathrm{O}\right)$ or iron chelator (EP) for 48 hours. For rescue, HSC were preloaded with $100 \mu \mathrm{g} / \mathrm{ml}$ of ferric ammonium citrate (FAC) for 1 hour at $37^{\circ} \mathrm{C}$ prior to iron chelator Cell staining and imaging: Cells were fixed with 4\% PFA for 10 min and permeabilized with $0.15 \%$ Triton X-100/PBS for 5 min at RT. Cells were then blocked in $2 \%$ BSA/0.15\% Triton X-100 in PBS for 1 hour at RT and incubated overnight at $4^{\circ} \mathrm{C}$ with the first antibody diluted in $1 \%$ BSA $0.15 \%$ Triton $\mathrm{X}-100$ followed by 45 min incubation at RT with fluorescence-conjugated secondary antibodies. Cells were washed 3 times with PBS and mounted with Prolong Gold (Molecular Probes) containing $1 \mu \mathrm{g} / \mathrm{ml}$ DAPI. All the images were acquired with a Confocal microscope (SP5, Leica) using 63.0x 1.40 NA oil objective and the Leica LAS-AF software. Image processing and analysis were performed using ImageJ (https://imagej.nih.gov/ij/). 


\section{Metabolomics}

1053 Metabolomic profiling and analysis were performed as previously described ${ }^{44}$. Ex vivo

1054 treated human CD34+ cells, as well as murine HPC-7 and LSK cells were flash-frozen in

1055 liquid nitrogen and stored at $-80^{\circ} \mathrm{C}$ before use. Samples were either sent to the Biological

1056 Mass Spectrometry Core Facility at University of Colorado Denver, or the Einstein-Mt

1057 Sinai Diabetes Center Stable Isotope \& Metabolomics Core for further processing and 1058 analysis.

1059 Cell pellets were lysed with lysis solution (methanol:acetonitrile:water 5:3:2 v/v/v) before 1060 ice cold extraction by vortexing for $30 \mathrm{~min}$ at $4^{\circ} \mathrm{C}$. Insoluble proteins were pelleted by 1061 centrifugation $\left(15,000 \times \mathrm{g}\right.$ for $10 \mathrm{~min}$ at $\left.4^{\circ} \mathrm{C}\right)$ and supernatants were analysed using a 1062 UHPLC system (Vanquish, ThermoFisher) coupled online to a mass spectrometer (Q 1063 Exactive, ThermoFisher). Samples were resolved over a Kinetex C18 column $(2.1 \times 150$ $1064 \mathrm{~mm}, 1.7 \mu \mathrm{m}$; Phenomenex) at $25^{\circ} \mathrm{C}$ using a 9-minute gradient at $400 \mu \mathrm{l} / \mathrm{min}$ from $5 \%$ to 1065 95\% B (A: water/0.1\% formic acid; B: acetonitrile/0.1\% formic acid). MS analysis and data 1066 analysis were performed as described before ${ }^{134,135}$. Metabolite assignments were 1067 performed using a metabolomics data analyser (MAVEN). For data analysis, the level of 1068 each metabolite was normalized to internal standard (heptadecanoic acid) and cell 1069 number. Differentially altered metabolites were submitted for pathway enrichment 1070 analysis using Hypergeometric test of MetaboAnalyst 3.0136. Moreover, differentially 1071 altered metabolites in each treatment condition were also analysed for biological function, 1072 upstream regulators, and canonical pathways using IPA.

\section{Seahorse assay}


1074 Oxygen consumption rates (OCR) were measured using a 96-well Seahorse Bioanalyzer 1075 XF 96 according to the manufacturer's instructions (Agilent Technologies). In brief, HPC-

10767 or Acs/4 knockdown BA/F3 cells were cultured in their normal growth media, exposed 1077 to either vehicle or iron chelator for 16 hours. DEUP (100 $\mu \mathrm{M}$ Diethylumbelliferyl 1078 phosphate, Sigma) was used to inhibit lipolysis. To determine the fraction of oxygen 1079 consumption dependent on fatty acid $\beta$-oxidation, cells $(75,000$ cells/well, with $3-4$ 1080 replicate wells) were plated into $180 \mu \mathrm{KHB}$ media (111 mM NaCl, $4.7 \mathrm{mM} \mathrm{KCl,} 1.25 \mathrm{mM}$ $1081 \mathrm{CaCl}$, $2 \mathrm{mM} \mathrm{MgSO} 4,1.2 \mathrm{mM} \mathrm{NaH2PO4})$ and in half of the samples etomoxir $(40 \mu \mathrm{M}$, 1082 Sigma, E1905) was added for 15-30 min before the analysis. Once in the reader, plates were sequentially injected with $1 \mu \mathrm{M}$ oligomycin, $2 \mu \mathrm{M}$ FCCP and $0.5 \mu \mathrm{M}$ Rotenone. Fatty 1084 acid $\beta$-oxidation rate was calculated as the difference in oxygen consumption in the 1085 presence or absence etomoxir. Data were normalized to cell number using trypan blue 1086 exclusion and analysis was performed with Waze v2.6.0.31.

\section{Acs/4 knockdown}

1088 Short hairpin RNAs (shRNAs) targeting Acs/4 (TL502838, OriGene) were packaged in 1089 the pGFP-C-shLenti plasmid system. A nontargeting 29-mer scrambled shRNA cassette 1090 in pGFP-C-shLenti vector (TR30021, OriGene) served as a control. The sequences of 1091 Acs/4 shRNAs can be found in Extended Data Table 11. For production of virus 1092 particles, lentiviral shRNA expression constructs were transfected together with 1093 packaging vectors (VSV-G, RSV-Rev and GAG-Pol) into 293T producer cells using 1094 CalPhos Transfection Kits (Takara Bio) following the manufacturer's instructions. 1095 Supernatants were harvested after 48 and 72 hours post transfection, and concentrated 1096 by ultracentrifugation $\left(25,000 \mathrm{rpm}\right.$ at $4^{\circ} \mathrm{C}$ for 2 hours). For cell lines, lentiviral transduction 
1097 was performed by adding the virus supernatant to BA/F3 cells and spin-infected for 90 $1098 \min$ at $1000 \times \mathrm{g}, 37^{\circ} \mathrm{C}$. After spin-infection, the cells were exposed to the virus for 2.5

1099 hours. Forty-eight hours post transduction, the percentage of transduced cells were analysed by FACS for GFP positivity. For primary mouse bone marrow cells, lineage-

1101 depleted MNC were enriched for c-Kit+ cells by magnetic-activated cell sorting (MACS)

1102 following manufacturer's protocol. Briefly, Lin- cells were re-suspended in $2 \%$ FBS/PBS 1103 and incubated with FcR blocking reagent for 10 min on ice and then mouse monoclonal 1104 CD117-microbeads (Miltenyi; 130-091-224) for $15 \mathrm{~min}$ on ice. Prior to lentiviral 1105 transduction, Lin-c-Kit+ (LK) cells were pre-cultured for 4 hours in M5300 media 1106 supplemented with $100 \mu \mathrm{g} / \mathrm{ml}$ primocin, $50 \mathrm{ng} / \mathrm{ml} \mathrm{rmSCF}, 50 \mathrm{ng} / \mathrm{ml} \mathrm{rmFlt3L,} 50 \mathrm{ng} / \mathrm{ml}$ $1107 \mathrm{rmTPO}$, and $2 \mathrm{ng} / \mathrm{ml} \mathrm{rmlL}-3$. Lentiviruses for shAcs/4 and scrambled nontargeting control 1108 were added to RetroNectin (TaKaRa)-coated 12-well plates and centrifuged for 2 hours 1109 at $1000 \times \mathrm{g}$ at $32^{\circ} \mathrm{C}$. After centrifugation, supernatant was discarded and pre-cultured LK 1110 cells were added immediately at a density of $500,000 \mathrm{cell} / \mathrm{ml}$. Thereafter, the cells were 1111 spin-infected for 2 hours at $1000 \times \mathrm{g}$ at $37^{\circ} \mathrm{C}$. After spin-infection the virus-containing 1112 media was removed and replenished with fresh M5300 media supplemented with 1113 cytokines. Forty-eight hours post transduction, GFP-positive cells were sorted on BD

1114 FACS Aria II (Becton Dickinson) and knockdown of Acsl4 was confirmed using qPCR and 1115 western blot.

\section{Western blot}

1117 To assess Acs/4 knockdown, sorted primary GFP-positive cells were analysed by 1118 Western blot. For HPC-7 cell analyses, cells treated with iron chelators or vehicle control, 
1119 protein expression changes were assessed for Acsl4, Cox1/Ptgs1, ferritin heavy (Fth1)

1120 and light (Flt1) chains.

1121 Proteins were extracted from cells using RIPA buffer (50 mM Tris, pH 7.4, 1 mM EDTA,

$1122150 \mathrm{mM} \mathrm{NaCl}, 1 \%$ Triton $\mathrm{X}-100,1 \%$ deoxycholate and $0.1 \%$ SDS) supplemented with

1123 EDTA-free protease inhibitor cocktail (Sigma, 11873580001) and $1 \mathrm{mM} \mathrm{PMSF.} \mathrm{Protein}$

1124 concentrations were determined using Protein Assay Kit (BioRad, 5000002). Prior to

1125 loading, cell extracts were mixed with appropriate volumes of $5 x$ protein loading buffer

1126 (10\% SDS, 25\% 2-Mercapoethanol, 50\% Glycerol, $125 \mathrm{mM}$ Tris-HCl pH 6.8, and 0.125\%

1127 Bromophenol blue) and boiled at $95^{\circ} \mathrm{C}$ for $10 \mathrm{~min}$. Subsequently, $20-30 \mu \mathrm{g}$ of total proteins

1128 from each sample were separated by SDS/polyacrylamide gel electrophoresis in running

1129 buffer (25 mM Tris pH 8.3, $192 \mathrm{mM}$ Glycine, 0.1\% SDS) and transferred onto

1130 nitrocellulose (NC) membranes (Bio-Rad, 1620094) in transfer buffer (25 mM Tris pH 8.3,

1131192 mM Glycine) under 300 mA constant current for respective period of time depending

1132 on the molecular weight of the target proteins. NC membranes were washed once with

1133 Tris-buffered saline-Tween 20 (TBST, $20 \mathrm{mM}$ Tris $\mathrm{pH}$ 8.3, $137 \mathrm{mM} \mathrm{NaCl,} \mathrm{0.1 \%} \mathrm{Tween}$

1134 20) and blocked in 5\% skim milk/TBST for 1 hour at RT. After washing three times with

1135 TBST, NC membranes were probed overnight at $4^{\circ} \mathrm{C}$ with the following primary

1136 antibodies: Acsl4 (Santa Cruz Biotech, sc-365230), Cox1/Ptgs1 (Cell Signaling

1137 Technologies, 4841), ferritin light chain (abcam, ab109373), ferritin heavy chain (abcam,

1138 ab65080) and actin (abcam, ab3280). Prior to and after the incubation with goat anti-

1139 rabbit (Santa Cruz Biotech, sc-2004) or goat anti-mouse (Santa Cruz Biotech, sc-2005)

1140 IgG-HRP diluted in 5\% skim milk/TBST at RT for 1 hour, NC membranes were washed 1141 with TBST for three times. All blots were developed using Pierce ${ }^{\mathrm{TM}}$ ECL Western Blotting 
1142 Substrate (Thermo Fisher Scientific, 32106). Signals were visualized and collected by LI-

1143 COR Odyssey Fc (LI-COR Biosciences). ImageJ (https://imagej.nih.gov/ij/) was used for

1144 quantification.

1145 ELISA

1146 For the preparation of serum, peripheral blood was collected from mice into non1147 anticoagulant-treated tube and left undisturbed at RT to clot for 30 minutes. Clot was

1148 removed by centrifuging at 2,000 RCF for 10 minutes in a refrigerated centrifuge, and 1149 supernatant was then transferred and aliquoted into $1.5 \mathrm{~mL}$ clean polypropylene tubes 1150 for storage at $-80^{\circ} \mathrm{C}$ until use. ELISA assay was performed with Mouse Transferrin ELISA 1151 kit (ab157724, Abcam, Waltham, MA) and Mouse Ferritin (FTL) ELISA kit (ab157713, 1152 Abcam) according to manufacturer's protocols. Briefly, serum sample was diluted 1:40, 1153 or 1:100,000 with $1 \mathrm{X}$ diluent for the quantification of ferritin or transferrin protein, 1154 respectively. Standard samples were prepared according to manufacturer's instructions.

$1155100 \mu \mathrm{L}$ diluted sample or standard control were transferred to 96 well plate strips and 1156 incubated in the dark at RT for 30 minutes. Thereafter, each well was emptied and 1157 washed four times with $200 \mu \mathrm{L} 1 \mathrm{X}$ Wash buffer. $100 \mu \mathrm{L}$ of $1 \mathrm{X}$ HRP-Antibody conjugate 1158 was then transferred to each well and incubated in the dark at RT for 30 minutes, followed 1159 by four washes with $1 \times$ Wash buffer. Thereafter, $100 \mu \mathrm{L}$ of TMB Substrate Solution was 1160 transferred to each well and incubated in the dark at RT for 10 minutes. Reaction was 1161 stopped by adding $100 \mu \mathrm{L}$ of Stop Solution to each well. Absorbance at $450 \mathrm{nM}$ was then 1162 measured with FLUOstar Omega Microplate reader (BMG, Cary, NC). The protein level 1163 was calculated using the standard curve of standard samples.

1164 Integrative analysis with published data sets 
1165 Full list of published data sets used for comparative analyses is available in Extended

Data Table 12. For microarray data sets (GSE93649, GSE78829 and GSE12538), CEL

1167 files were retrieved from Gene Expression Omnibus (GEO) database. Gene expression

1168 signals across samples were normalized with RMA algorithm, and differential expression

1169 analysis was performed with R package limma ${ }^{126}$. For RNA-seq data sets (GSE120705),

1170 sequencing reads were retrieved from Sequence Read Archive (SRA) database with SRA

1171 Toolkit 2.10.7. After the removal of adapter contamination and low-quality reads with Trim

1172 Galore v0.6.5 (https://github.com/FelixKrueger/TrimGalore), gene expression

1173 quantification was performed with Salmon 1.2.1 ${ }^{137}$. Differential expression analysis was

1174 performed with tximeta 1.6.2 and DESeq2 1.28.0138,139. For single cell RNA-seq data sets

1175 (GSE70657 and GSE59114), matrix of gene counts was obtained from GEO database.

1176 Normalization and differential expression were then performed with DESeq2 $1.28 .0^{139}$.

1177 For chromatin immunoprecipitation sequencing (ChIP-seq) data sets (GSE49847,

1178 GSE69671, GSE34483, GSE39237, GSE16256, GSE43103, GSE59636, GSE47082,

1179 GSE43007, GSE47765, GSE76055, and GSE22075), binding peaks and gene targets

1180 were retrieved from Cistrome Data Browser. Only genes with regulatory potential score >

11810.5 were used as targets for further analysis. For data sets not available in Cistrome Data

1182 Browser (GSE120705), sequencing reads were retrieved from SRA database with SRA

1183 Toolkit. Following the analytical pipeline used in Cistrome, ChiLin workflow was used for

1184 peaking calling and gene target prediction ${ }^{140}$. Briefly, after the removal of adapter 1185 contamination and low-quality reads with Trim Galore, genome alignment (mm10) was

1186 performed with BWA $0.7 .17^{141}$. Unambiguously mapped sequencing reads were 1187 subjected to peak calling with MACS2 2.1.1 ${ }^{142}$. Thereafter, target gene analysis was 
1188 performed with BETA 1.0.7 to examine the regulatory potential for each RefSeq genes ${ }^{143}$.

1189 Binding targets with regulatory potential score $>0.5$ were used as targets for further

1190 analysis. For comparative analyses performed with the data sets, the significance $p$ -

1191 values for the overlapping genes were calculated by hypergeometric test with built-in

1192 phyper function of $\mathrm{R}$.

1193 Statistical analysis

1194 All results were expressed as the mean values \pm s.e.m. unless otherwise noted. Prism8

1195 (www.graphpad.com) was used for statistical analyses with Student's $t$-tests. Unless

1196 otherwise specified, all statistical tests were two-sided, and analyses for significant

1197 differences between two groups of paired and unpaired samples were conducted using

1198 paired and unpaired Student's $t$-test, respectively. Two-sample Kolmogorov-Smirnov

1199 tests were performed with built-in ks.test function of $\mathrm{R}$, to compare the difference of

1200 frequency distribution of Tfrc transcript per cell under different conditions. Hypergeometric

1201 tests were performed with built-in phyper function of R, to assess the enrichment of IRE-

1202 containing genes and the significance of gene set overlapping. Differential expression

1203 analysis with microarray data was performed with Limma Bioconductor package ${ }^{144}$.

1204 Differential expression analysis with RNA-seq data was performed with DESeq2 ${ }^{139}$.

1205 Enrichment analyses of pathways, and upstream regulators were conducted using

1206 NetworkAnalyst ${ }^{145}$, and Ingenuity Pathway Analysis (IPA). Enrichment analyses of gene

1207 sets were performed with GSEA for scRNA-seq data of iron chelator-treated HSC

1208 (GSEAPreranked analysis) and microarray data of Fbx/5-knockout HSC. Pre-ranked

1209 gene list for iron chelator-treated HSC were genes expressed in $>10 \%$ of all the single

1210 cells in the scRNA-seq data, and ranked by the fold changes in iron chelator treatment 
1211 compared to control. Signature score in single HSC was calculated with AddModuleScore

1212 module of Seurat package using the expression matrix of scRNA-seq. Estimation of stem

1213 cell frequency and significant differences in stem cell frequency between different groups

1214 in LTC-IC assays were estimated with ELDA method in the R package statmod ${ }^{146}$.

1215 Data availability

1216 Metabolomic data collected have been included in Extended Data Tables 6-9 in the

1217 Supplementary Information File. Transcriptomic data was deposited in GEO with 1218 accession numbers GSE157817 (Microarray gene expression profiling of mouse HSC 1219 treated with iron chelator deferoxamine (DFO)) and GSE157821 (Single cell RNA-seq of 1220 mouse HSC treated with iron chelator deferoxamine (DFO)).

\section{Acknowledgements}

1222 We thank the members of the Will lab for feedback on and discussions of the study, and

1223 Amit Verma for feedback on the manuscript; Sofiya Milman, Derek Huffman and Nir

1224 Barzilai for very helpful discussions, access to aged mice and Longenity cohort data. We

1225 further thank Daqian Sun and Swathi-Rao Narayanagari from the Stem Cell Isolation and

1226 Xenotransplantation Core and Jinghang Zhang at the Einstein Cell Sorting Core facilities

1227 for technical assistance with FACS analysis and cell sorting; David Reynolds from the

1228 Einstein Genomics Core facility for single cell capture for RNA-seq, Yunping Qiu and 1229 Xueliang Du at the Einstein-Mt Sinai Diabetes Center Stable Isotope \& Metabolomics

1230 Core, the Einstein Analytical Imaging Facility, the Einstein Institute for Animal Studies, 1231 and Research Facilities Coordinator Peter Schultes for expert support and technical 1232 assistance. We also thank Justin Wheat and Goichi Tatsumi for intellectual and technical 1233 support for the smRNA FISH experiments. This study was supported by grants from the 
1234 National Institutes of Health P30CA013330 (core support grant), DK105134 and 1235 CA230756 (to B.W.), as well as investigator-initiated research projects sponsored by

1236 GlaxoSmithKline and Novartis Pharmaceuticals (to B.W.), and a Pershing Square Sohn

1237 Prize for Young Investigators in Cancer Research (to B.W.). Y.R.K. is supported by a T32

1238 Training grant in Aging Research (AG023475; PI: Barzilai) and M.M.A. is supported by

1239 the Einstein Training Program in Stem Cell Research (NYSTEM C30292GG; PI:

1240 Frenette). R.K. is the recipient of a Career Development Program Postdoctoral Fellowship

1241 from the Leukemia \& Lymphoma Society (LLS).

\section{Author contributions}

1243 Y.R.K. and J.C. designed, performed, analysed and interpreted the cell functional,

1244 molecular, imaging and FACS-based assays, designed and conducted in silico data 1245 mining, and wrote the manuscript; R.K. established and performed all smRNA FISH

1246 analyses; R.K. analysed, and R.K. and U.S. interpreted the smRNA FISH data.; M.T. 1247 assisted with primary cell culture model set-up and data collection; Y.M. performed

1248 Western blot analyses; M.M.A. performed the Asc/4 KD model set up and validation, and

1249 Ascl4 KD LTC-IC data collection; A.Z., V.T. and M.T. performed mouse colony 1250 management, dissection and harvest of hematopoietic cells from the bone marrow of 1251 animals; S.S.L. and J.H. performed and collected data for mass spectrometry-based 1252 proteomic and metabolomic analyses; S.S. and A.D'A. coordinated and interpreted mass

1253 spectrometry-based proteomic and metabolomic analyses; B.W. designed and 1254 coordinated the study, contributed to designing the experiments and interpreting the data, 1255 and wrote the manuscript. 
1257 B.W. has received funds for research projects and serving on advisory boards from

1258 Novartis Pharmaceuticals.

1259 Additional information

1260 Supplementary Information is available for this paper.

1261 Correspondence and requests for materials should be addressed to Yun-Ruei Kao,

1262 Ph.D. (yun-ruei.kao@einsteinmed.org) or Britta Will, Ph.D. (britta.will@einsteinmed.org).

\section{Method References}

1265

1266

1267

1268

1269

1270

1271

1272

1273

1274

1275

1276

1277

1278

1279

1280

1281

1282

1283

1284

1285

1286

1287

1288

1289

1290

111 Pinto do, O. P., Kolterud, A. \& Carlsson, L. Expression of the LIM-homeobox gene LH2 generates immortalized steel factor-dependent multipotent hematopoietic precursors. EMBO J 17, 5744-5756 (1998).

112 Palacios, R. \& Steinmetz, M. II-3-dependent mouse clones that express B-220 surface antigen, contain Ig genes in germ-line configuration, and generate $B$ lymphocytes in vivo. Cell 41, 727-734 (1985).

113 Thomas, F. et al. Calcein as a fluorescent probe for ferric iron. Application to iron nutrition in plant cells. J Biol Chem 274, 13375-13383 (1999).

114 Prus, E. \& Fibach, E. Flow cytometry measurement of the labile iron pool in human hematopoietic cells. Cytometry A 73, 22-27 (2008).

115 Hershberg, E. A. et al. PaintSHOP enables the interactive design of transcriptome- and genome-scale oligonucleotide FISH experiments. bioRxiv, 2020.2007.2005.188797 (2020).

116 Maekiniemi, A., Singer, R. H. \& Tutucci, E. Single molecule mRNA fluorescent in situ hybridization combined with immunofluorescence in S. cerevisiae: Dataset and quantification. Data Brief 30, 105511 (2020).

117 Loeffler, D. et al. Mouse and human HSPC immobilization in liquid culture by CD43- or CD44-antibody coating. Blood 131, 1425-1429 (2018).

$118 \mathrm{Hu}, \mathrm{Y}$. \& Smyth, G. K. ELDA: extreme limiting dilution analysis for comparing depleted and enriched populations in stem cell and other assays. J Immunol Methods 347, 70-78 (2009).

119 Leenen, P. J. et al. Differential inhibition of macrophage proliferation by anti-transferrin receptor antibody ER-MP21: correlation to macrophage differentiation stage. Exp Cell Res 189, 55-63 (1990).

120 Goodwin, J. M. et al. Autophagy-Independent Lysosomal Targeting Regulated by ULK1/2-FIP200 and ATG9. Cell Rep 20, 2341-2356 (2017). 
1291121 Portilla, D. et al. Etomoxir-induced PPARalpha-modulated enzymes protect during acute

1292

1293

1294

1295

1296

1297

1298

1299

1300

1301

1302

1303

1304

1305

1306

1307

1308

1309

1310

1311

1312

1313

1314

1315

1316

1317

1318

1319

1320

1321

1322

1323

1324

1325

1326

1327

1328

1329

1330

1331

1332

1333

1334 renal failure. Am J Physiol Renal Physiol 278, F667-675 (2000).

122 Lopaschuk, G. D., Wall, S. R., Olley, P. M. \& Davies, N. J. Etomoxir, a carnitine palmitoyltransferase I inhibitor, protects hearts from fatty acid-induced ischemic injury independent of changes in long chain acylcarnitine. Circ Res 63, 1036-1043 (1988).

123 Huang, M. J., Cheng, Y. C., Liu, C. R., Lin, S. \& Liu, H. E. A small-molecule c-Myc inhibitor, 10058-F4, induces cell-cycle arrest, apoptosis, and myeloid differentiation of human acute myeloid leukemia. Exp Hematol 34, 1480-1489 (2006).

124 Gao, C. et al. Rational design and validation of a Tip60 histone acetyltransferase inhibitor. Sci Rep 4, 5372 (2014).

125 Carvalho, B. S. \& Irizarry, R. A. A framework for oligonucleotide microarray preprocessing. Bioinformatics 26, 2363-2367 (2010).

126 Ritchie, M. E. et al. limma powers differential expression analyses for RNA-sequencing and microarray studies. Nucleic acids research 43, e47 (2015).

127 Campillos, M., Cases, I., Hentze, M. W. \& Sanchez, M. SIREs: searching for ironresponsive elements. Nucleic Acids Res 38, W360-367 (2010).

128 O'Leary, N. A. et al. Reference sequence (RefSeq) database at NCBI: current status, taxonomic expansion, and functional annotation. Nucleic Acids Res 44, D733-745 (2016).

129 Zheng, G. X. et al. Massively parallel digital transcriptional profiling of single cells. Nat Commun 8, 14049 (2017).

130 Giladi, A. et al. Single-cell characterization of haematopoietic progenitors and their trajectories in homeostasis and perturbed haematopoiesis. Nat Cell Biol 20, 836-846 (2018).

131 Tikhonova, A. N. et al. The bone marrow microenvironment at single-cell resolution. Nature 569, 222-228 (2019).

132 Will, B. et al. Satb1 regulates the self-renewal of hematopoietic stem cells by promoting quiescence and repressing differentiation commitment. Nat Immunol 14, 437-445 (2013).

$133 \mathrm{Du}, \mathrm{W}$. et al. Transferrin receptor specific nanocarriers conjugated with functional 7peptide for oral drug delivery. Biomaterials 34, 794-806 (2013).

134 Reisz, J. A., Zheng, C., D'Alessandro, A. \& Nemkov, T. Untargeted and Semi-targeted Lipid Analysis of Biological Samples Using Mass Spectrometry-Based Metabolomics. Methods Mol Biol 1978, 121-135 (2019).

135 Nemkov, T., Reisz, J. A., Gehrke, S., Hansen, K. C. \& D'Alessandro, A. High-Throughput Metabolomics: Isocratic and Gradient Mass Spectrometry-Based Methods. Methods Mol Biol 1978, 13-26 (2019).

136 Xia, J., Sinelnikov, I. V., Han, B. \& Wishart, D. S. MetaboAnalyst 3.0--making metabolomics more meaningful. Nucleic Acids Res 43, W251-257 (2015).

137 Patro, R., Duggal, G., Love, M. I., Irizarry, R. A. \& Kingsford, C. Salmon provides fast and bias-aware quantification of transcript expression. Nat Methods 14, 417-419 (2017).

138 Love, M. I. et al. Tximeta: Reference sequence checksums for provenance identification in RNA-seq. PLoS Comput Biol 16, e1007664 (2020).

139 Love, M. I., Huber, W. \& Anders, S. Moderated estimation of fold change and dispersion for RNA-seq data with DESeq2. Genome biology 15, 550 (2014). 
1335140 Qin, Q. et al. ChiLin: a comprehensive ChIP-seq and DNase-seq quality control and analysis pipeline. BMC Bioinformatics 17, 404 (2016). $\mathrm{Li}, \mathrm{H}$. \& Durbin, R. Fast and accurate short read alignment with Burrows-Wheeler transform. Bioinformatics 25, 1754-1760 (2009).

1339 Zhang, Y. et al. Model-based analysis of ChIP-Seq (MACS). Genome biology 9, R137

1340 (2008).

143 Wang, S. et al. Target analysis by integration of transcriptome and ChIP-seq data with BETA. Nature protocols 8, 2502-2515 (2013).

144 Ritchie, M. E. et al. limma powers differential expression analyses for RNA-sequencing and microarray studies. Nucleic Acids Research 43 (2015).

145 Zhou, G. et al. NetworkAnalyst 3.0: a visual analytics platform for comprehensive gene expression profiling and meta-analysis. Nucleic acids research 47, W234-W241 (2019). and enriched populations in stem cell and other assays. Journal of Immunological Methods 347, 70-78 (2009).

147 Laurenti, E. et al. Hematopoietic stem cell function and survival depend on c-Myc and NMyc activity. Cell Stem Cell 3, 611-624 (2008). proteins as master regulators of primary transcription response in leukemia. Sci Adv $\mathbf{2}$, e1600760 (2016). 
bioRxiv preprint doi: https://doi.org/10.1101/2021.08.03.454947; this version posted August 5, 2021. The copyright holder for this preprint (which was not certified by peer review) is the author/funder, who has granted bioRxiv a license to display the preprint in perpetuity. It is made available under aCC-BY-NC-ND 4.0 International license.

\section{Extended data figures}

\section{Extended data figure 1}

a

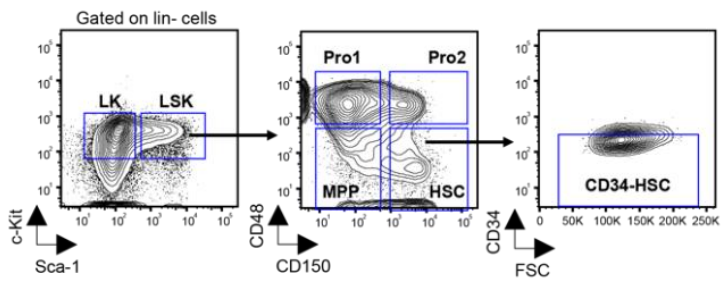

C

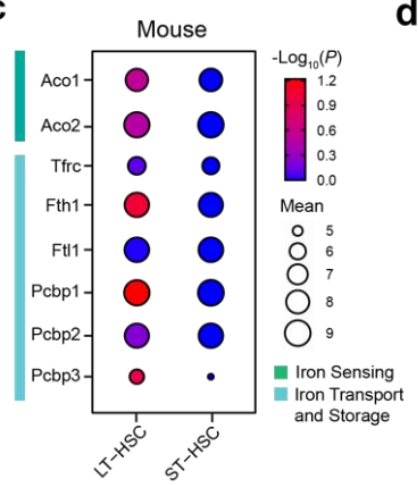

d

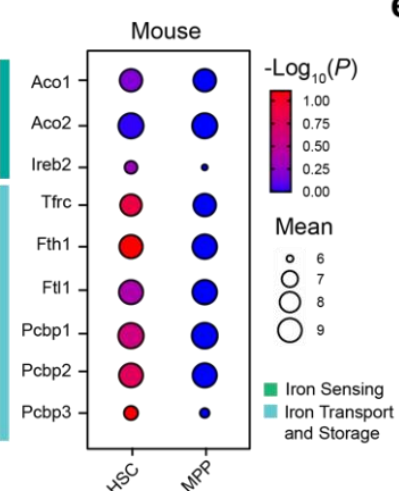

g

f
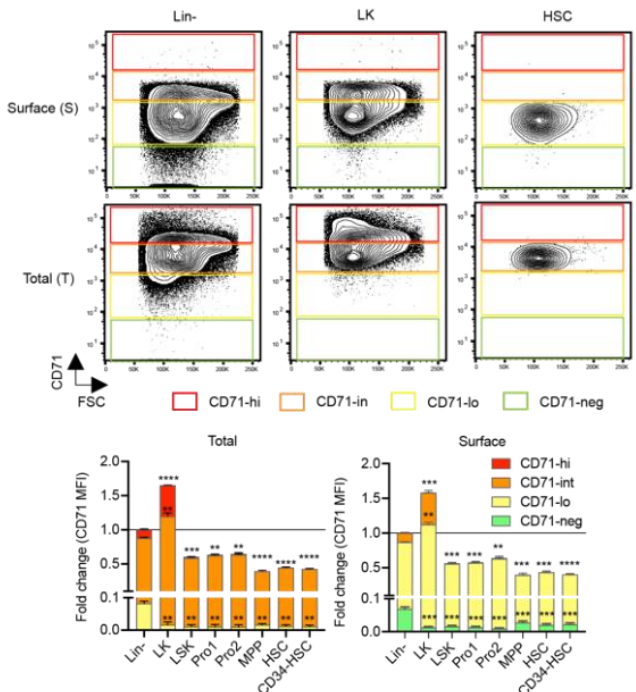

k

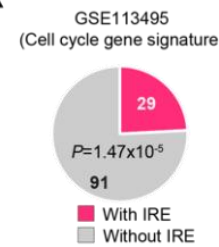

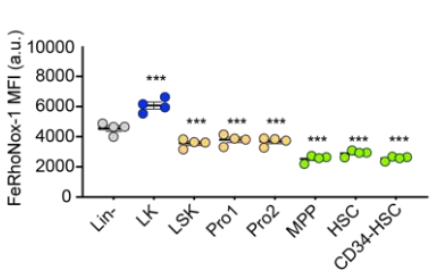

e
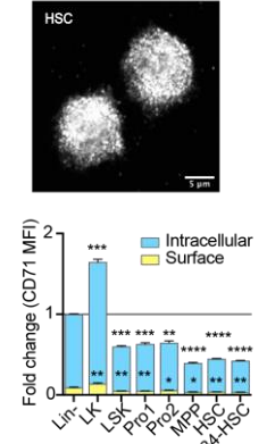

b
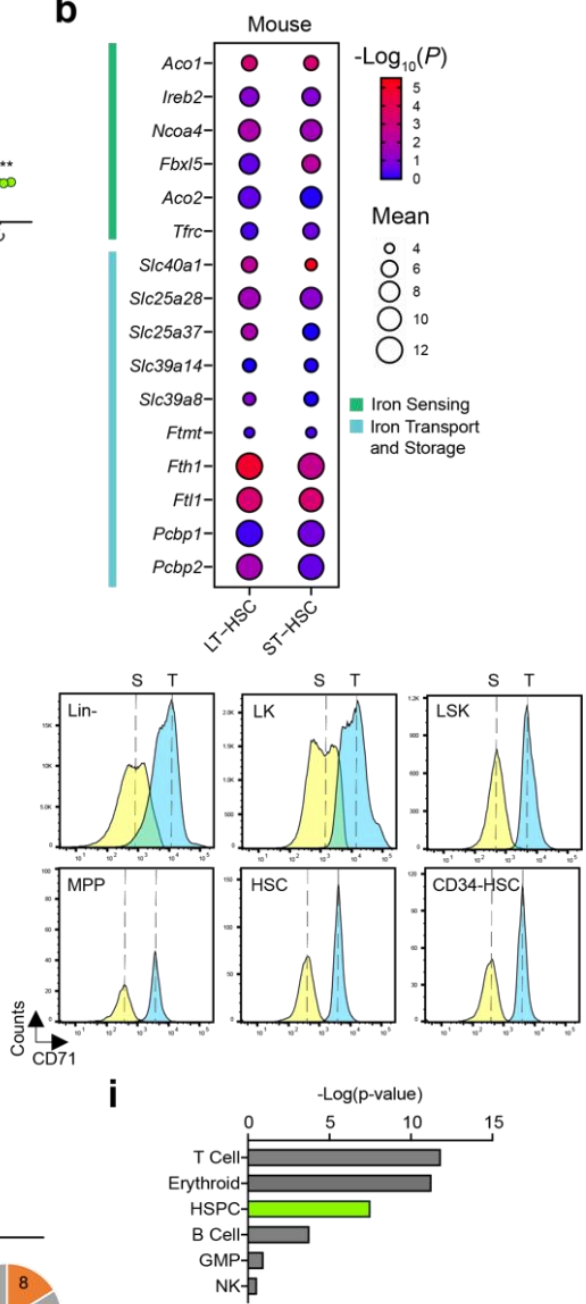

h

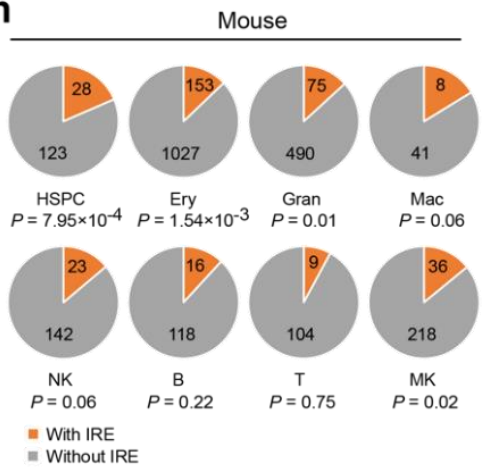


bioRxiv preprint doi: https://doi.org/10.1101/2021.08.03.454947; this version posted August 5, 2021. The copyright holder for this preprint (which was not certified by peer review) is the author/funder, who has granted bioRxiv a license to display the preprint in perpetuity. It is made available under aCC-BY-NC-ND 4.0 International license.

\section{Extended data figure 2}

a

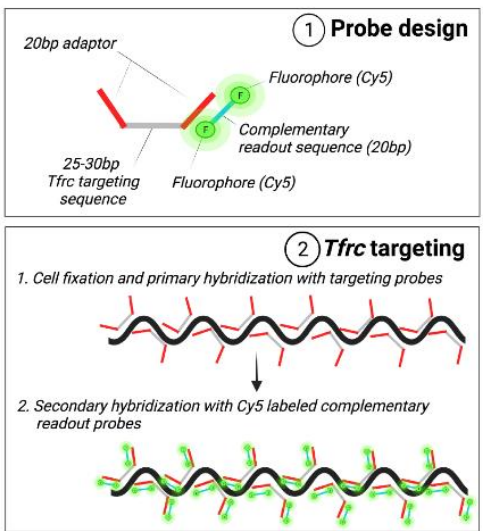

d

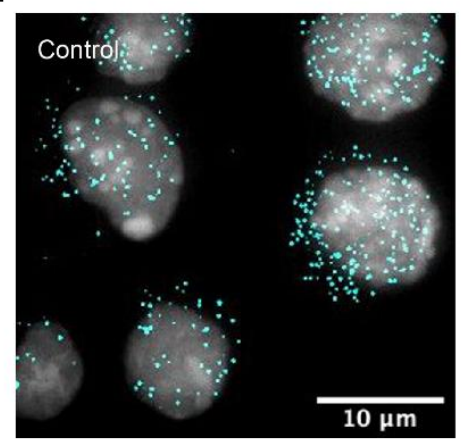

h

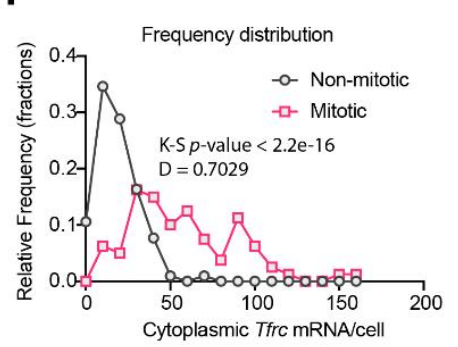

I

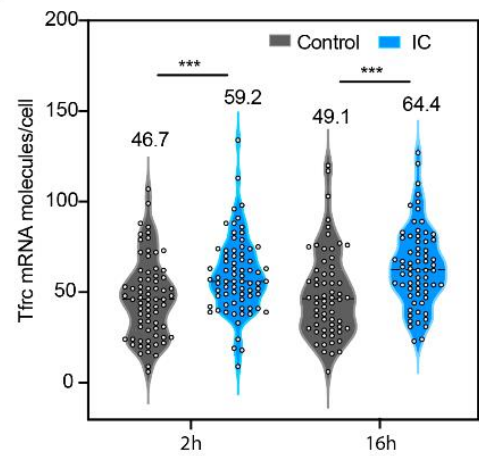

e

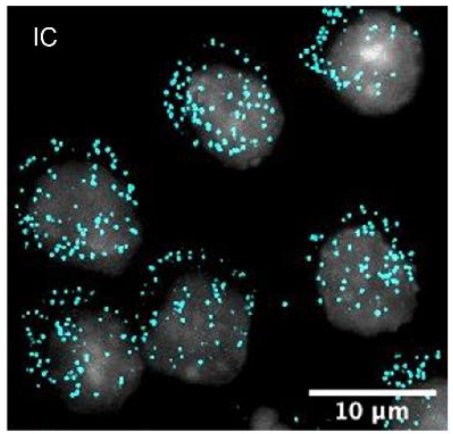

i

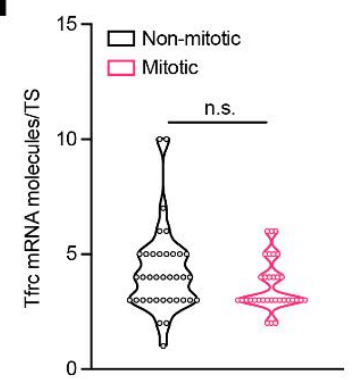

m

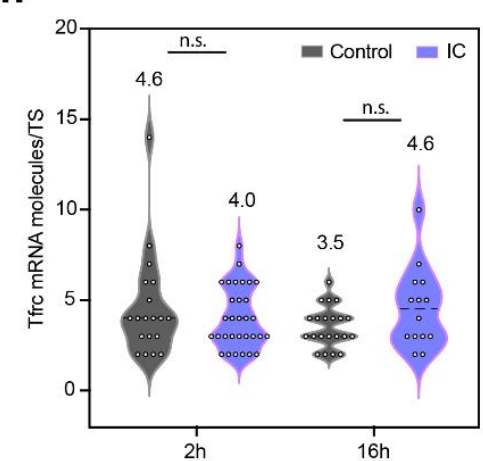

f b

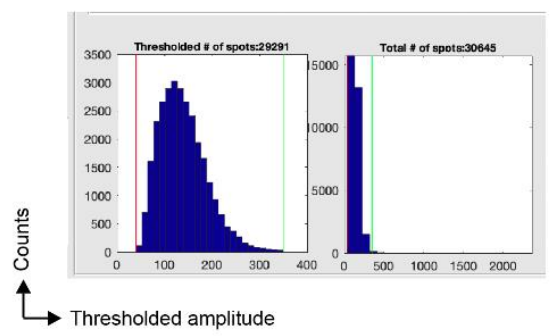

C
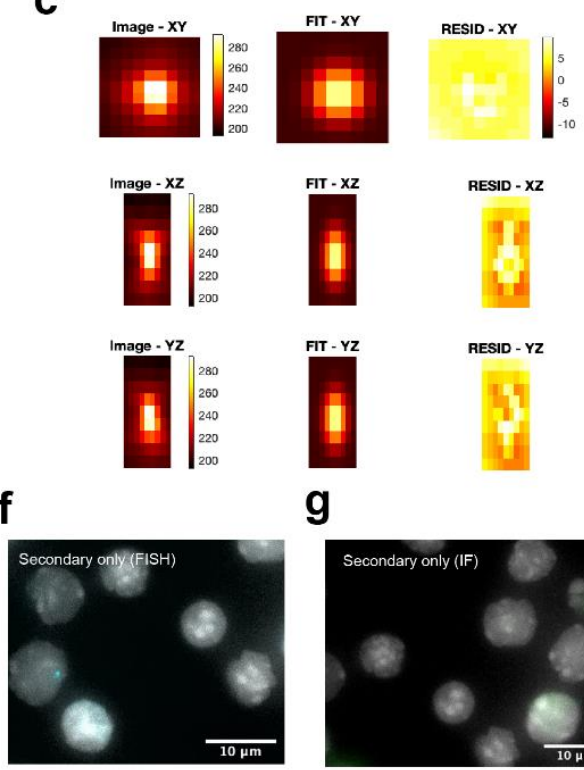

g

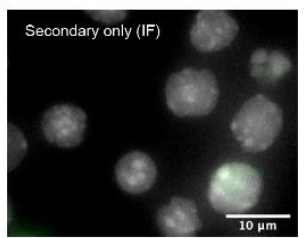

k
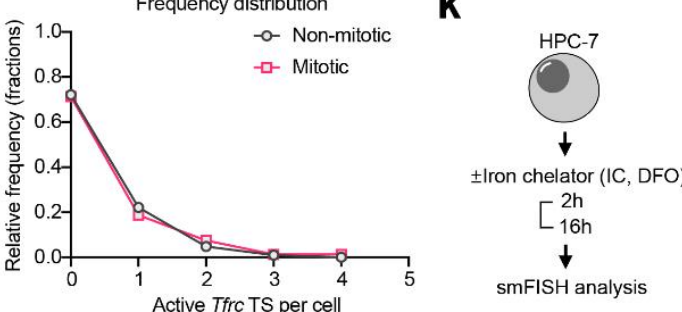

n

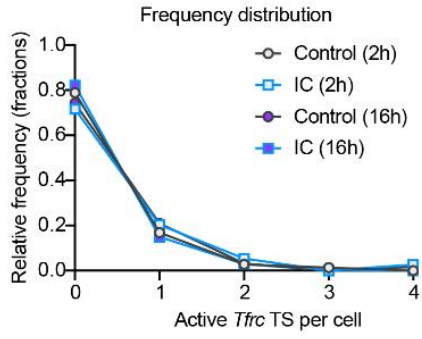


bioRxiv preprint doi: https://doi.org/10.1101/2021.08.03.454947; this version posted Auqust 5, 2021. The copyright holder for this preprint (which was not certified by peer review) is the author/funder, who has granted bioRxiv a license to display the preprint in perpetuity. It is made available under aCC-BY-NC-ND 4.0 International license.

\section{Extended data figure 3}

a
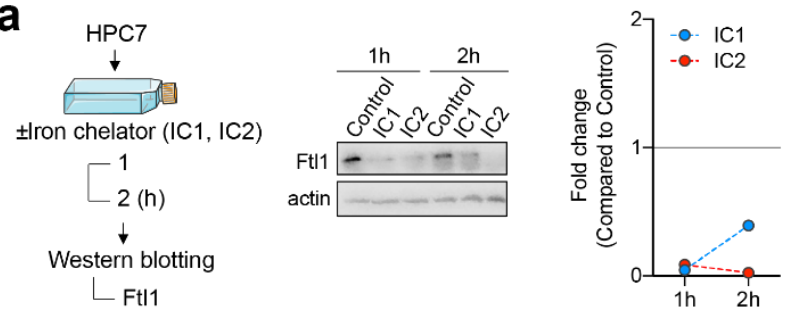

C

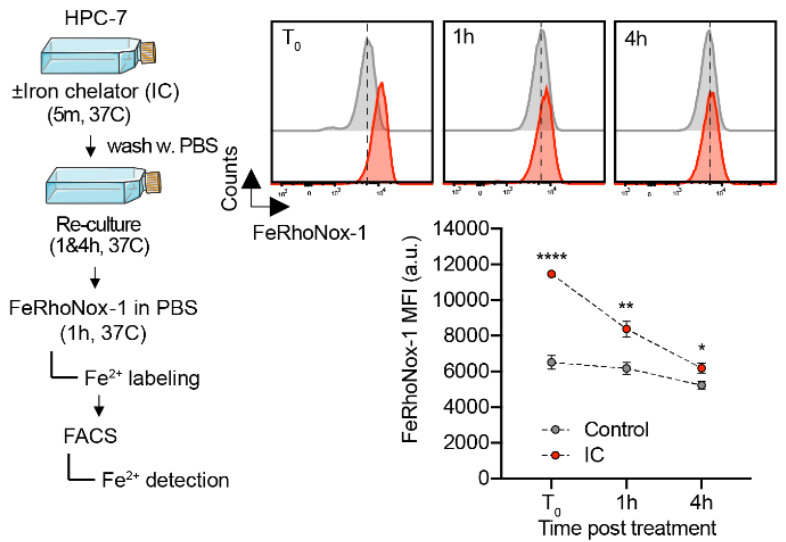

b

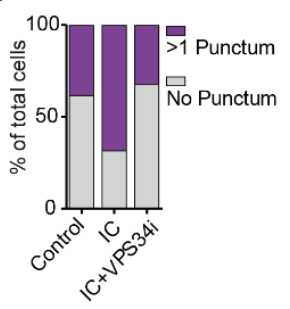

d

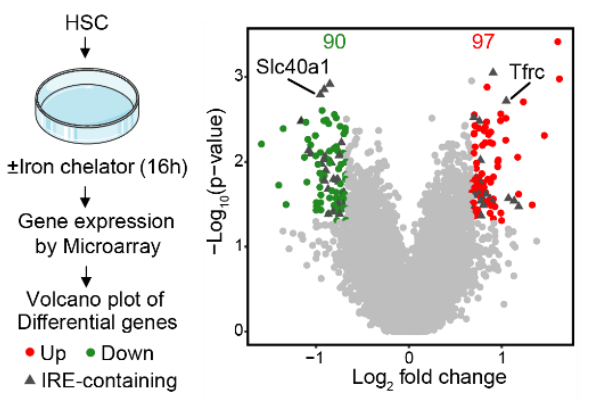

e

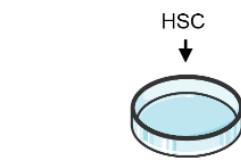

\pm Iron chelator (IC,16h)

$\downarrow$

Microarray (IRE enrichment analysis) qRT-PCR (Iron regulatory genes)

Apical Loop OC8

$\bigcirc$ GU/UG Pairing $\bigcirc$ Bulge/Missmatch
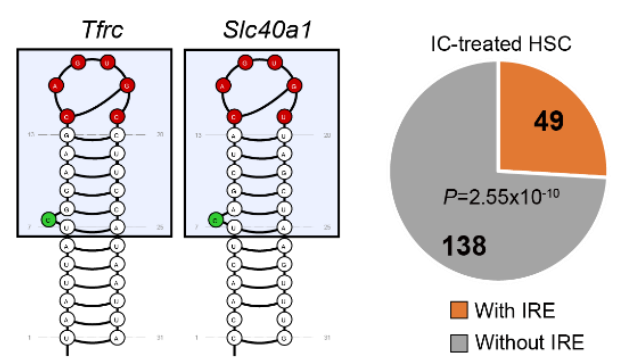

f
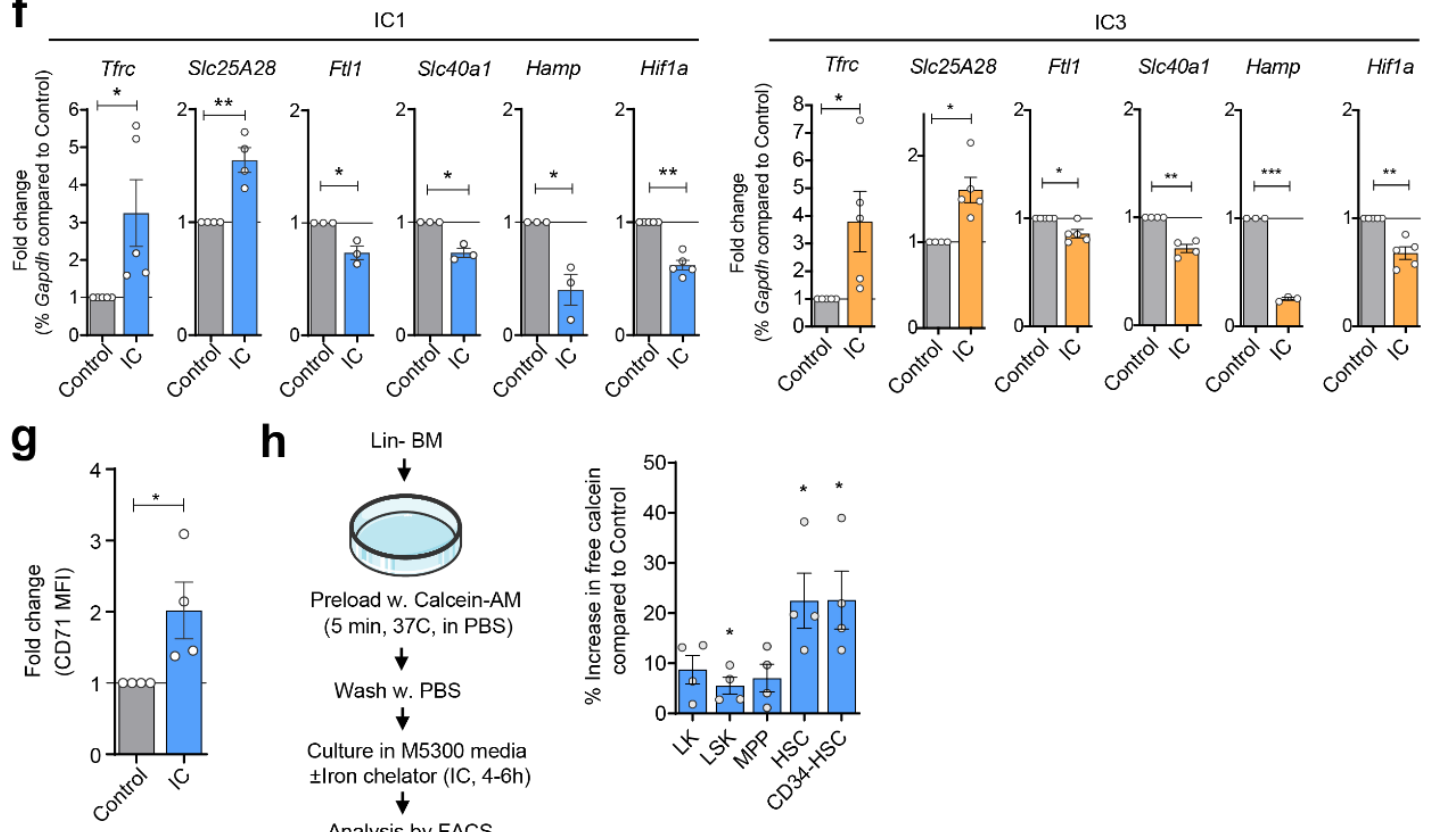

h
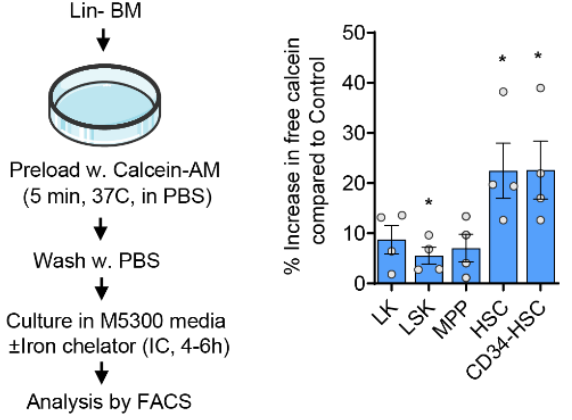


\section{Extended data figure 4}
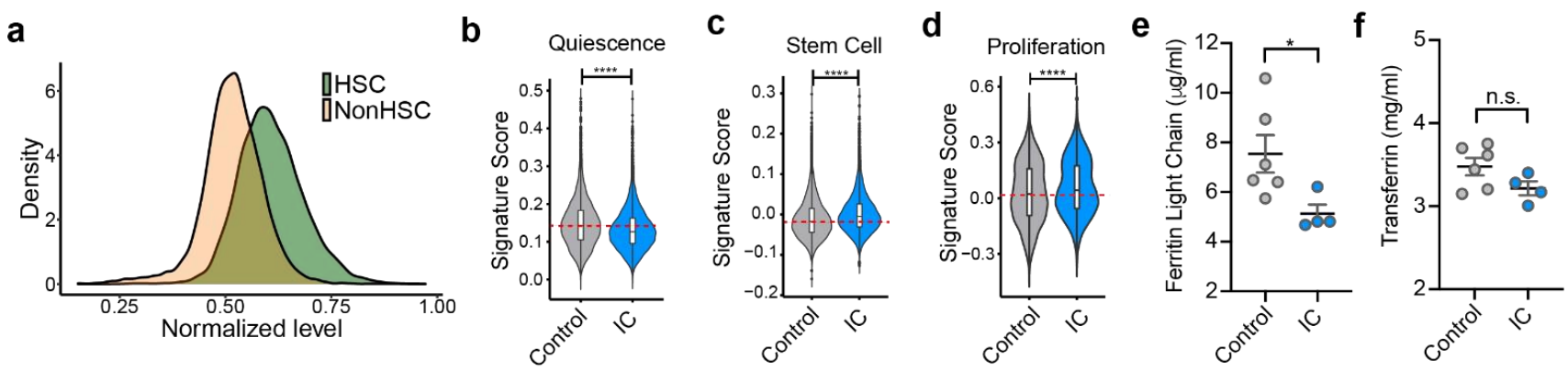

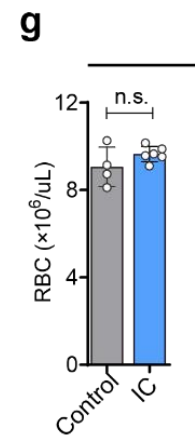

CBC
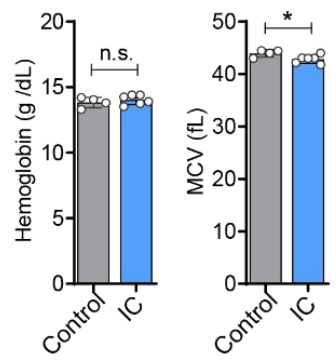

i

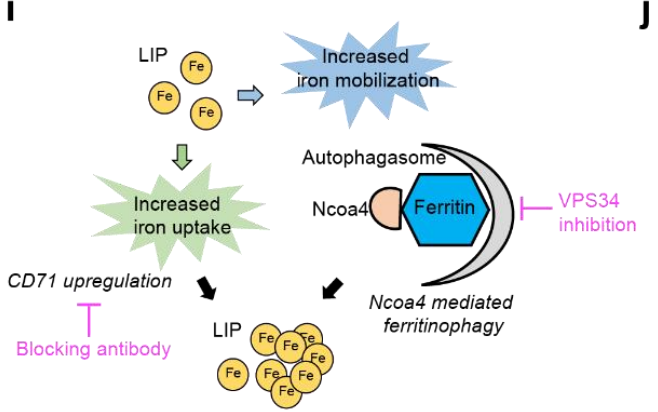

h
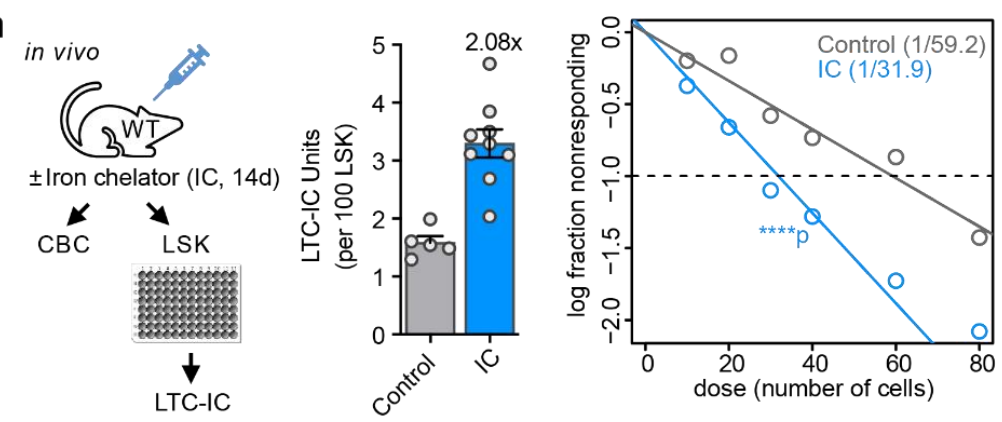

k

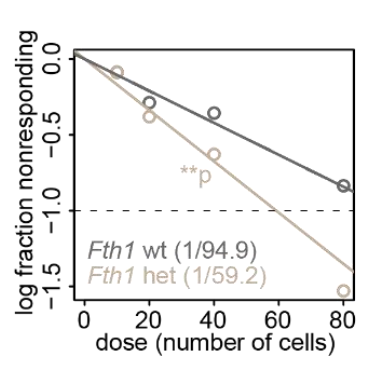

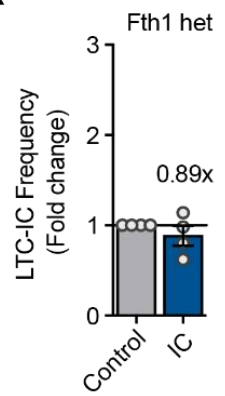

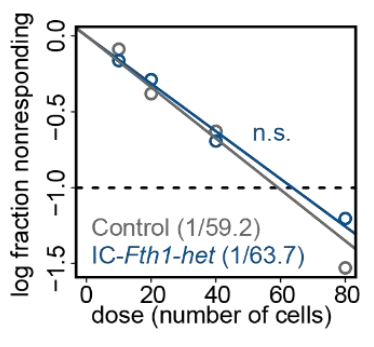


bioRxiv preprint doi: https://doi.org/10.1101/2021.08.03.454947; this version posted August 5, 2021. The copyright holder for this preprint (which was not certified by peer review) is the author/funder, who has granted bioRxiv a license to display the preprint in perpetuity. It is made available under aCC-BY-NC-ND 4.0 International license.

\section{Extended data figure 5}

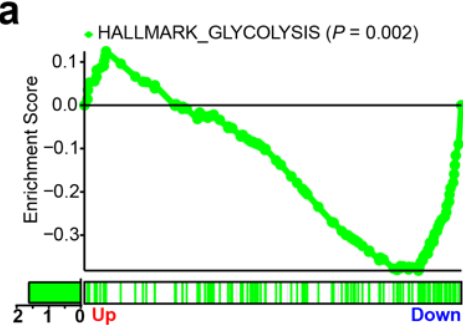

e

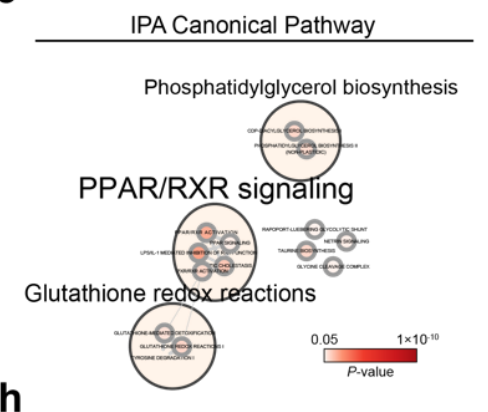

h

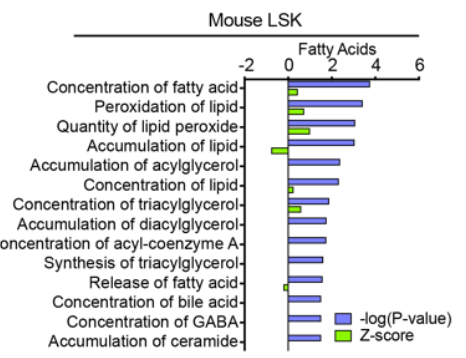

j

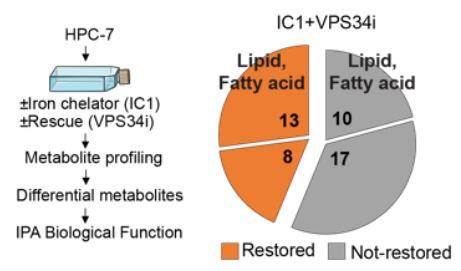

$\mathbf{k}$

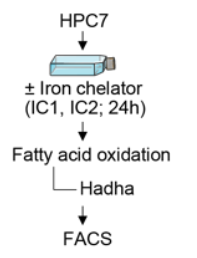

0
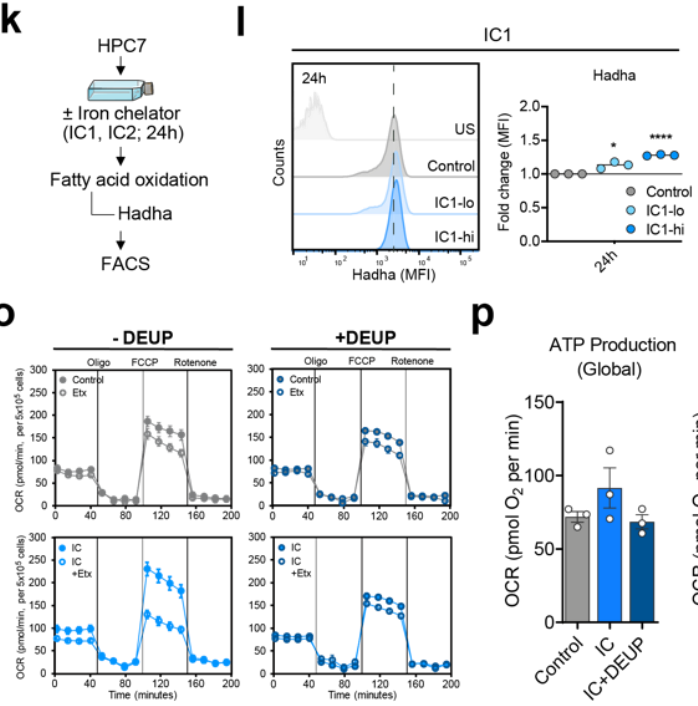

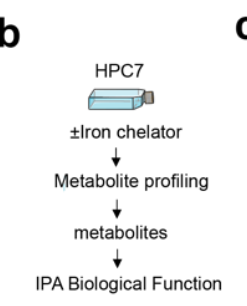

f

$$
\text { f }
$$

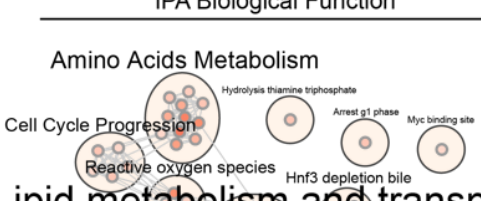

Lipid metabolism and transport

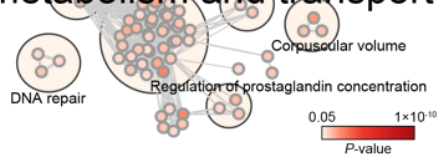

i

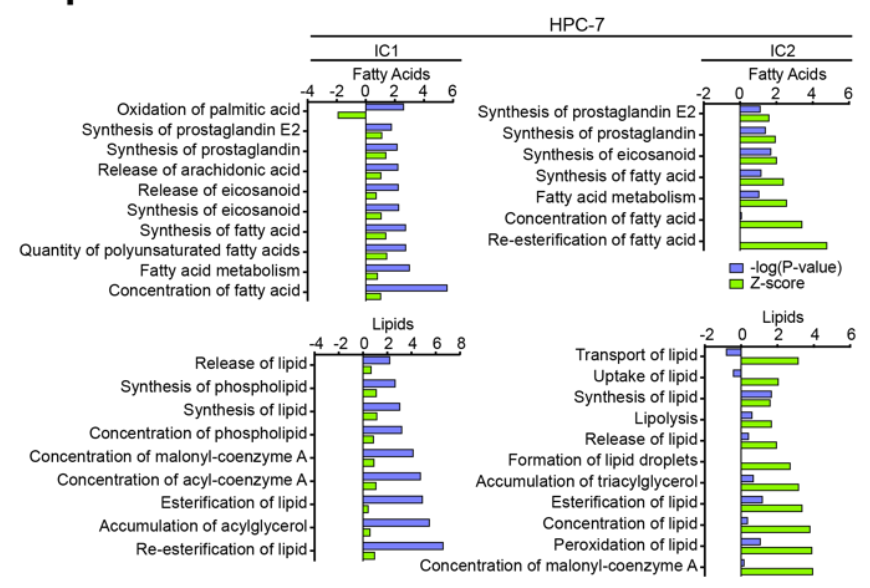

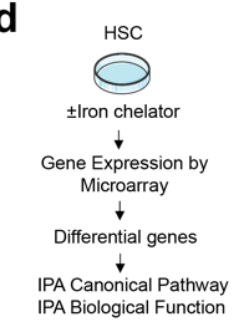

g

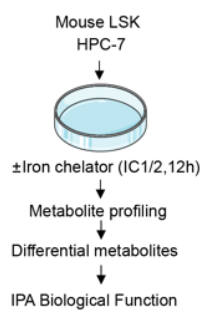

m

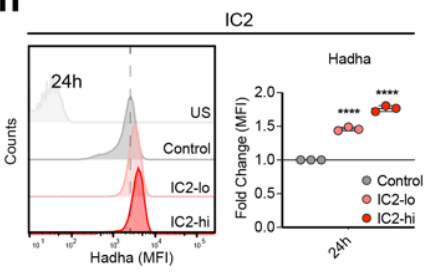

q

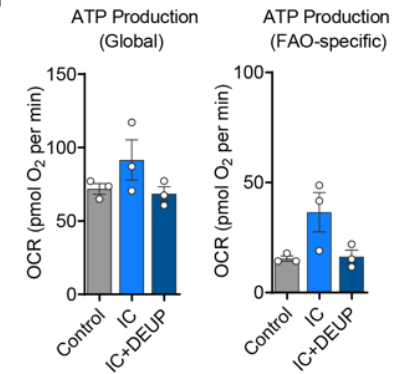

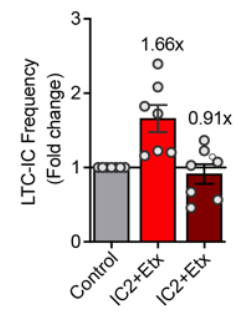
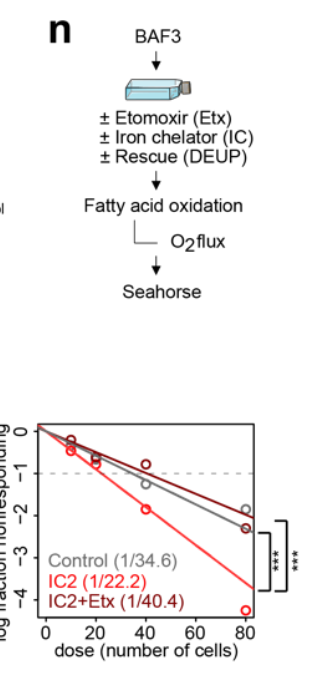


\section{Extended data figure 6}

a
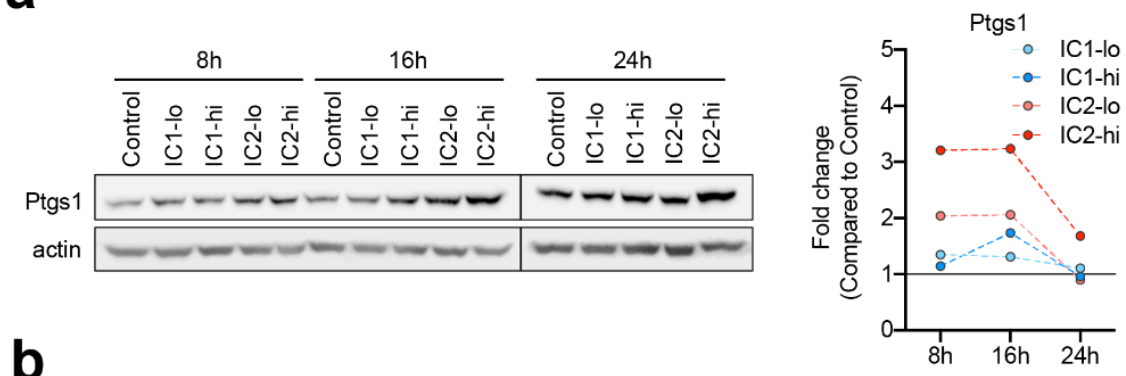

b

Haas S et al., Cell Stem Cell 2015
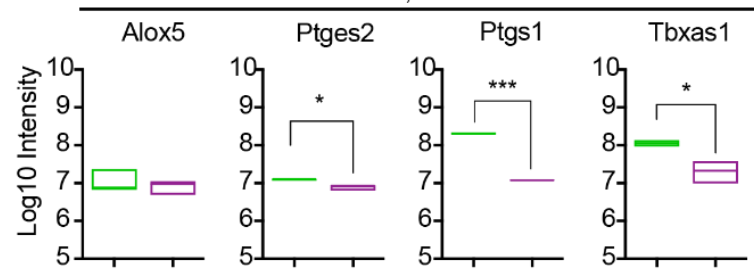

C

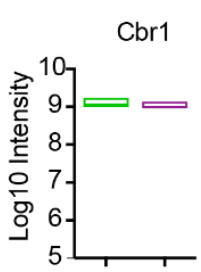

Ptges3
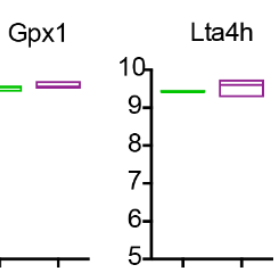

$\square$ HSC (Lin-Sca-1+cKit+CD150+CD48-)

$\square$ Prog (Lin-Sca-1+cKit+CD150+CD48+)

$\mathbf{e}$

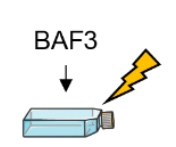

Acs 14 knockdown

- Targeted (KD)

- NTC

$\downarrow$

\pm Iron chelator (IC, 16h)

\pm Etomoxir (Etx, 15 min)

Fatty acid oxidation

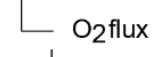

$\downarrow$

Seahorse
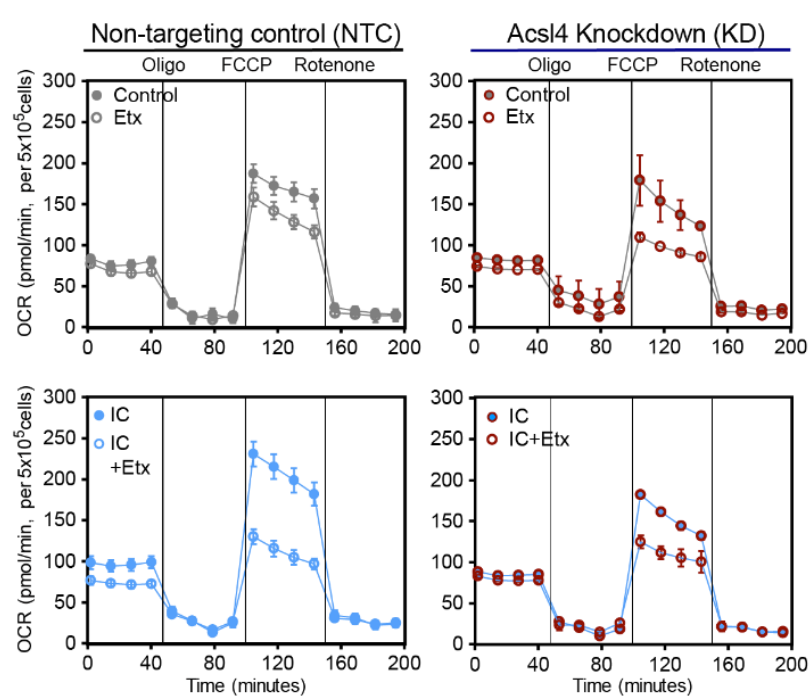

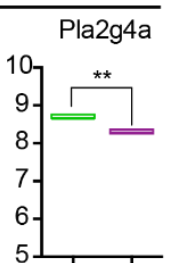

d

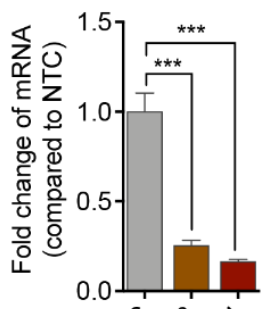

$95 \mathrm{kDa}$

$55 \mathrm{kDa}$

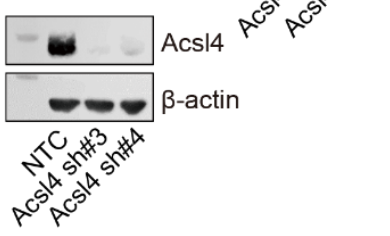

f Steady State

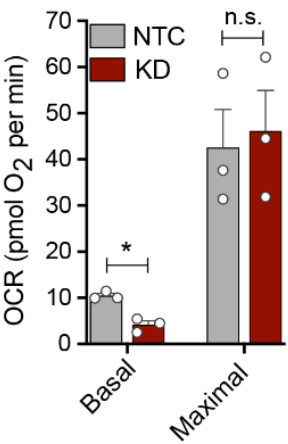


bioRxiv preprint doi: https://doi.org/10.1101/2021.08.03.454947; this version posted August 5, 2021. The copyright holder for this preprint (which was not certified by peer review) is the author/funder, who has granted bioRxiv a license to display the preprint in perpetuity. It is made available under aCC-BY-NC-ND 4.0 International license.

\section{Extended data figure 7}

1363

a

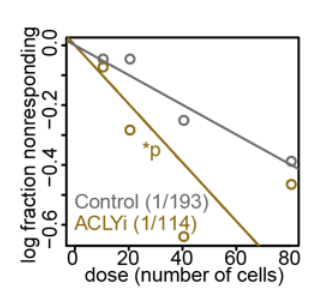

e

f

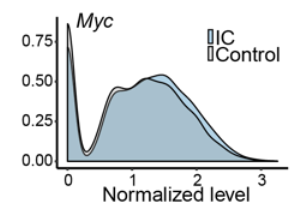

- MYCACTIV PATHWAY $(P<0.001)$
- MYC REPRESS PATHWAY $(P=0.04$

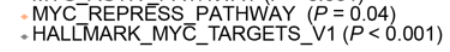

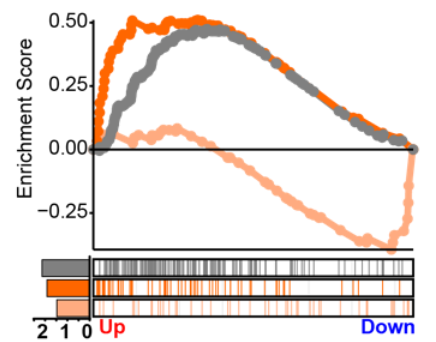

j

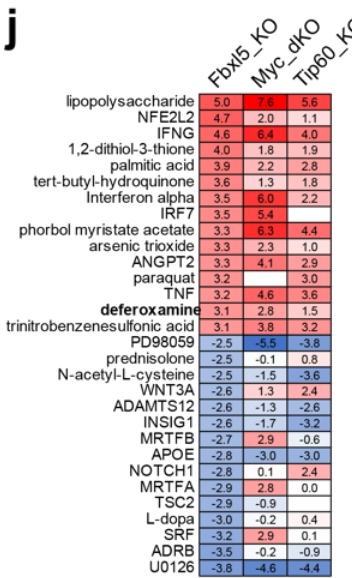

m

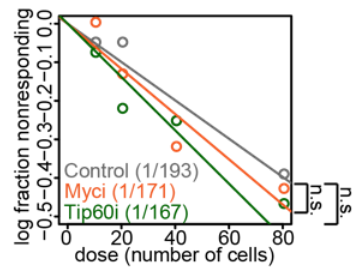

b

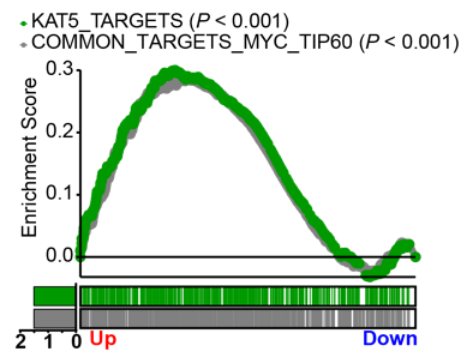

g

IC-treated HSC Myc-dKO HSC

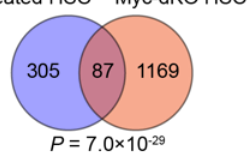

MYC_DKO HSC UP $(P<0.001)$

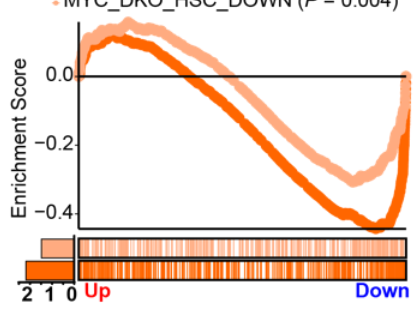

\section{k}

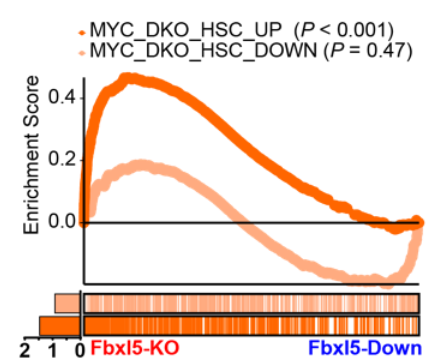

C

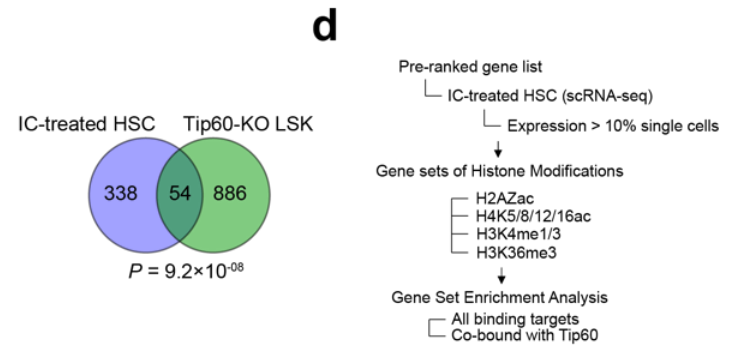

$\mathbf{h}$

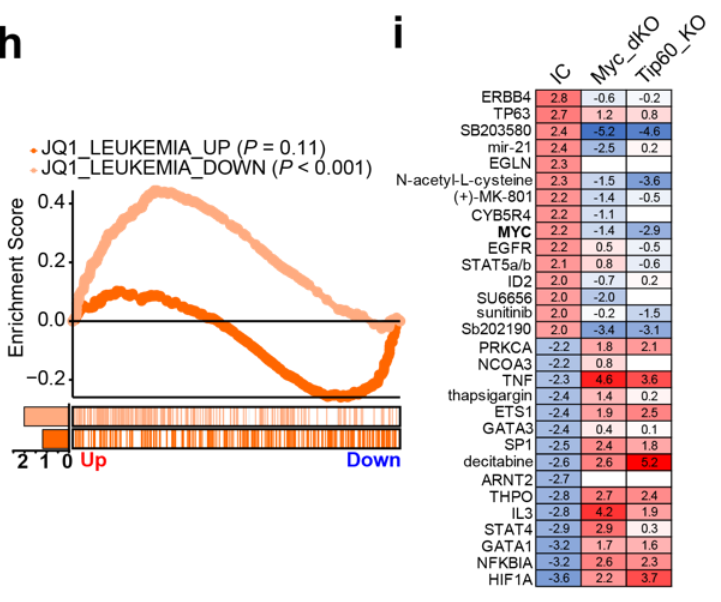

I

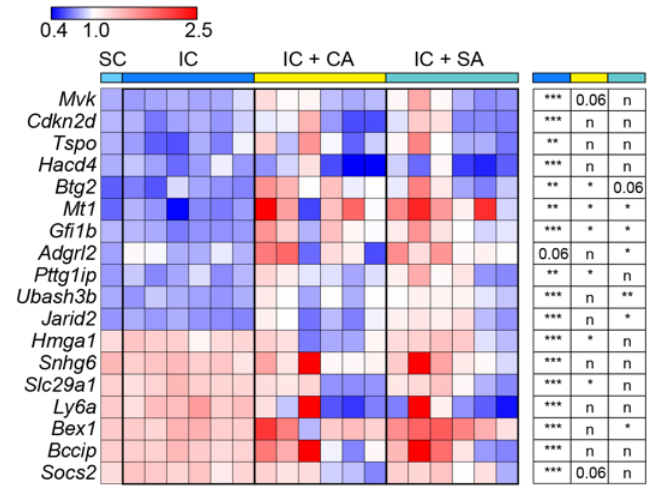

n

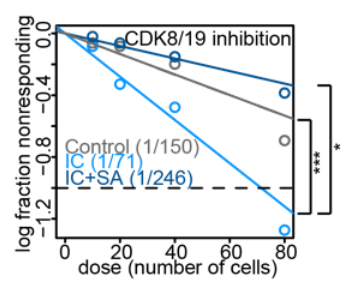

0

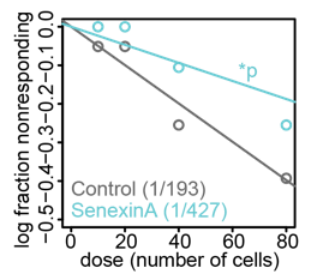


bioRxiv preprint doi: https://doi.org/10.1101/2021.08.03.454947; this version posted August 5, 2021. The copyright holder for this preprint (which was not certified by peer review) is the author/funder, who has granted bioRxiv a license to display the preprint in perpetuity. It is made available under aCC-BY-NC-ND 4.0 International license.

\section{Extended data figure 8}

a

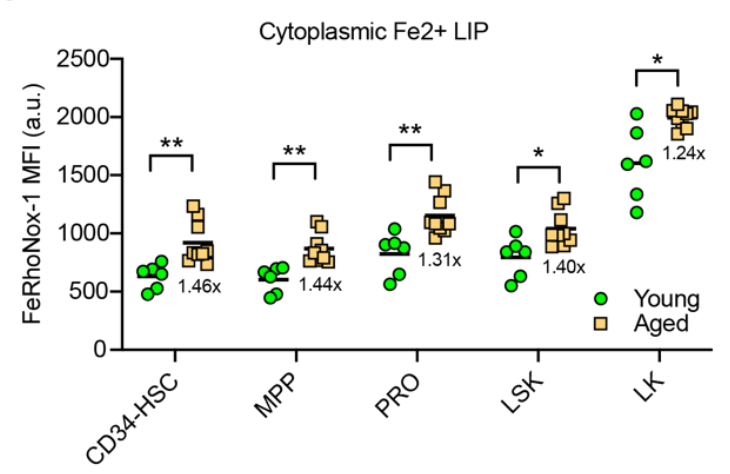

C

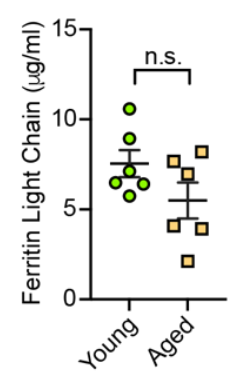

d

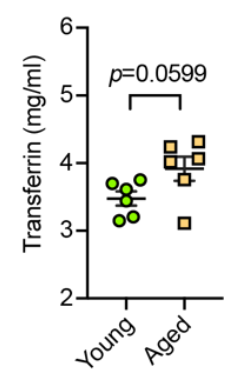

h TfrC

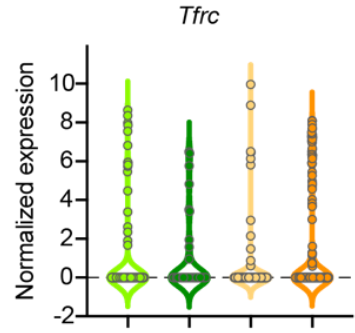

i

e

Platelet activation
signaling \& aggregation
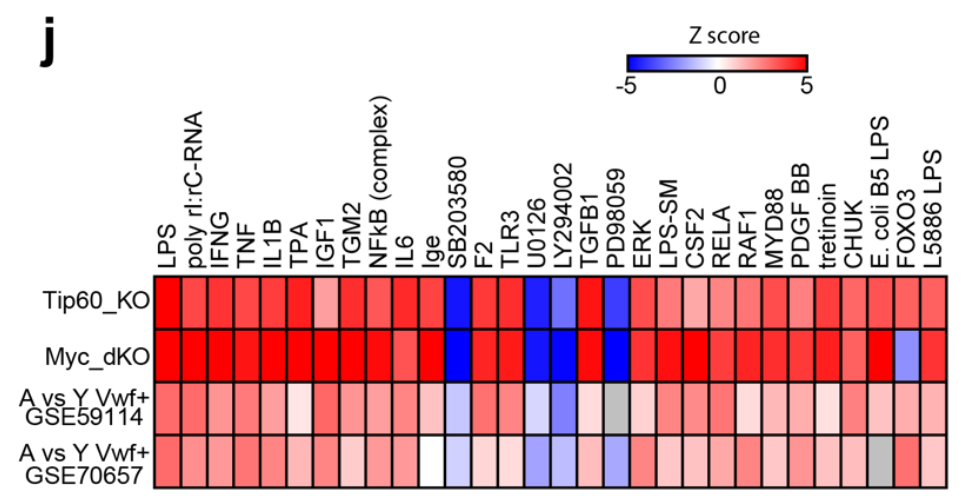

b

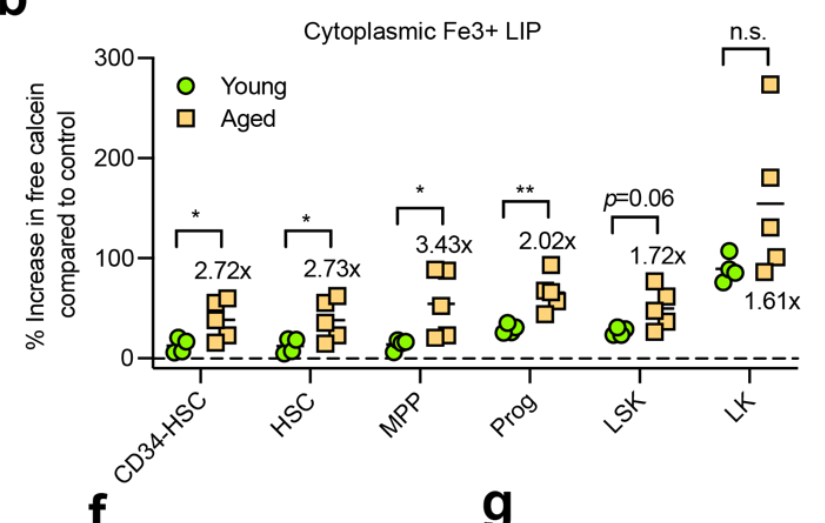

Mouse Aged HSC
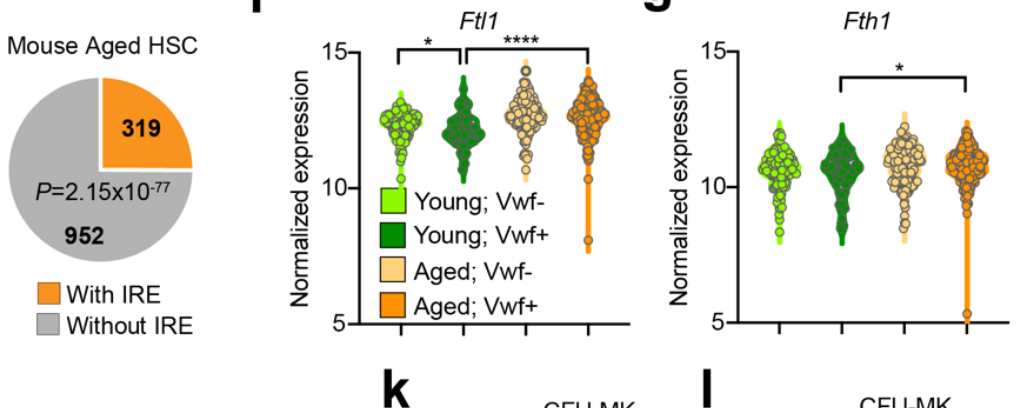

K

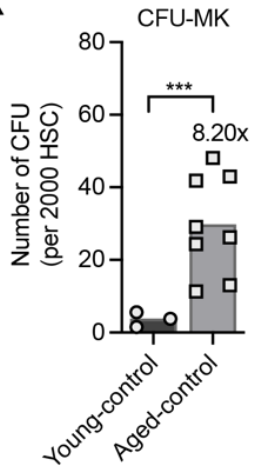

m

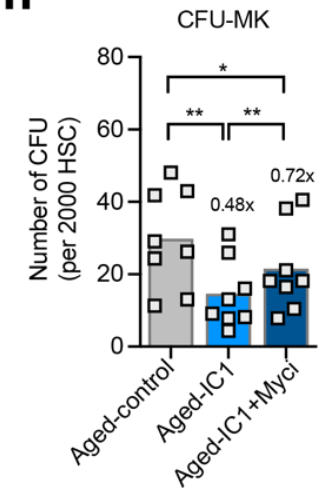

Cell surface interactions
at the vascular wall

at the vascular wall

n

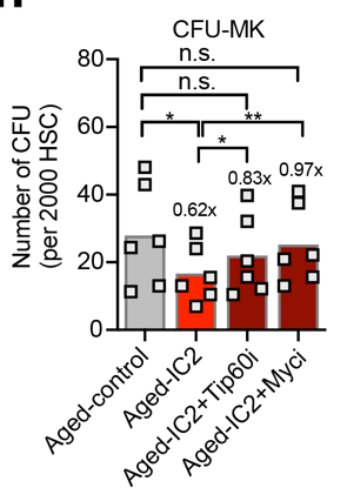




\section{Extended Data Figure 9}
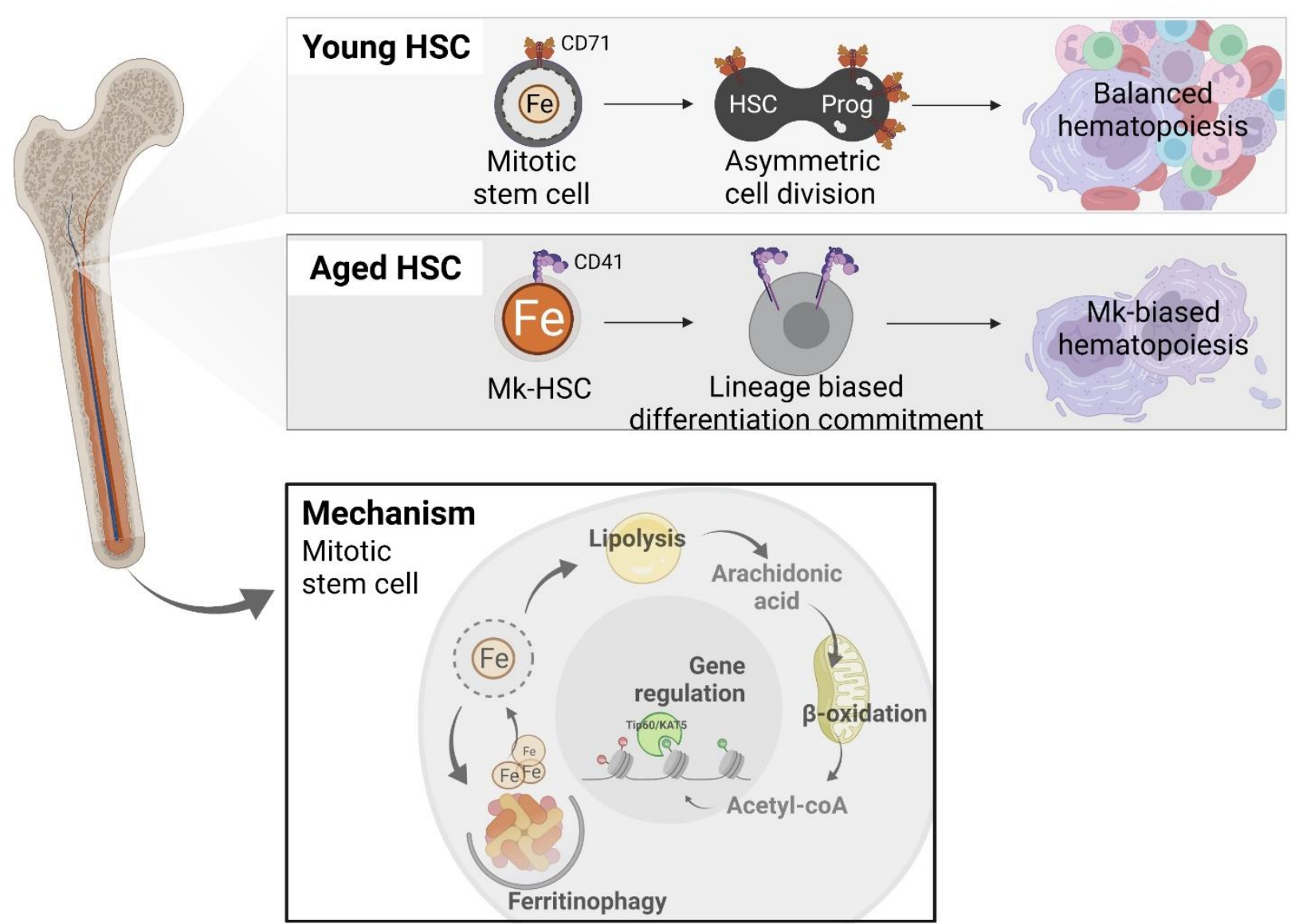


\section{Extended data figure/table legends}

1367 Extended Data Figure 1. Iron-dependent regulation of hematopoietic stem cell defining 1368 gene expression.

1369 a, Quantification of the intracellular labile iron pool (LIP) using FeRhoNox-1 and FACS analysis. 1370 Upper panel: Gating strategy for hematopoietic stem and progenitor cell (HPSC) populations; 1371 lower graph: Quantification of FeRhoNox-1 mean fluorescence intensity (MFI in arbitrary units 1372 (a.u.)). $\mathrm{n}=4$.

1373 b, mRNA expression of iron homeostasis-regulatory genes in HSC compared to progenitor cells (PMID: 18371395). Expression of genes with regulatory functions in iron sensing or transport and storage were examined. The normalized $\log _{2}$ expression values of relevant genes in LT-HSC or ST-HSC were compared to megakaryocyte-erythroid progenitor (MEP), $n=2-5$.

c,d, Protein expression of iron homeostasis-regulatory genes in murine HSPC (c: PMID25158935; d: PMID26299573). The levels of proteins with regulatory functions in iron sensing or transport and storage were examined. The $\log _{10}$ peptide intensities across HSC and progenitor cells were compared. $n=3$. Significance $p$-value were calculated using unpaired Student's t-test by comparing LT-HSC versus ST-HSC (c), or HSC versus MPP (d).

e, CD71 detection in purified HSC by immunofluorescence and confocal microscopy and quantification of cell surface and total CD71 content in HSPC populations by FACS analysis. The fold changes of CD71 MFI in different hematopoietic stem and progenitor cells compared to Linpopulation are shown, $\mathrm{n}=4$.

f, CD71 cell surface (S) and total (T) protein abundance in phenotypically defined hematopoietic stem and progenitor cell populations. Representative FACS plots for Lineage-negative ( Lin $^{-}$), LincKit'Sca-1- (LK) and Lin-ckit-Sca-1+CD48-CD150+ (HSC) cells (upper) and quantification (bottom). CD71-negative (CD71-neg), CD71-low (CD71-lo), CD71-intermediate (CD71-int), and CD71-high (CD71-hi) fractions were shown. Quantification presented as fold change of CD71 mean fluorescence intensity (MFI) in each cell populations compared to Lin $^{-}$cells for total CD71 protein levels (left graph) and cell surface presentation (right graph), $n=4$.

g,h, IRE enrichment analysis in murine hematopoietic cell type-specific gene expression signatures previously defined (g, GSE77098). h, Pie charts show the number of analyzed transcripts containing at least one IRE motif (With IRE, orange) or lacking it (Without IRE, gray). Hypergeometric test was used to calculate the significance $p$-value of IRE enrichment. signatures previously defined (GSE24759).

1399 k IRE enrichment analysis of genes associated with HSC cycling as previously defined (k, 1400 PMID29915358/GSE113495).

1401 If not specified differently, data are mean \pm SEM. $(\mathbf{a}, \mathbf{e}, \mathbf{f})$. Significance $p$-values, indicated as ${ }^{*} p$ $1402<0.05,{ }^{* *} p<0.01,{ }^{* *} p<0.001,{ }^{* * *} p<0.0001$, were calculated by Student's $t$-test (paired: a, e, 1403 f; unpaired: $\mathbf{b}, \mathbf{c}, \mathbf{d})$, or Hypergeometric test $(\mathbf{g}-\mathbf{j})$.

1404

1405 Extended Data Figure 2. Tfrc single mRNA molecule analysis in HSPC.

1406 a, Scheme showing sequential smRNA FISH procedure for Tfrc. 
b, Computational thresholding of amplitude of Tfre mRNA.

1408

1409

1410

1411

1412

1413

1414

1415

1416

1417

1418

1419

1420

1421

1422

1423

1424

1425

1426

1427

1428

1429

1430

1431

1432

1433

1434

1435

1436

1437

1438

1439

1440

1441

1442

1443

1444

1445

c, Fit of averaged image of Tfrc mRNA with 3D Gaussian distribution. First row: maximum intensity projections in $\mathrm{XY}$; second row: maximum intensity projections in $\mathrm{XZ}$. The first column shows the image, the second column the best fit, and the third column the absolute residuals.

$\mathbf{d}-\mathbf{g}$, Representative filtered and overlaid images of cells stained by smRNA FISH for Tfrc (Cy5, cyan pseudocolor), Control (d, HPC-7) vs. IC-treated (e, HPC-7), as well as secondary probe only controls for smRNA FISH (f, RO2-Cy5, Lin-cKit+ (LK) cells) and immunofluorescence (g, antirabbit AlexaFluor 488, LK cells) are shown. Nucleus is stained with DAPI (gray pseudocolor). Scale bars, $10 \mu \mathrm{m}$.

h, Histogram showing frequency distribution of cytoplasmic Tfrc mRNA per cell in non-mitotic and mitotic HSC (HPC-7).

i, Violin plot showing Tfrc nascent mRNA molecules per transcription site (TS) in non-mitotic $(n=36)$ and mitotic HSC $(n=34)$.

j, Histogram showing frequency distribution of active Tfrc transcription sites (TS) per cell in nonmitotic and mitotic HSC.

k, Schematic showing experimental flow for HPC-7 cells exposed to either control $\left(\mathrm{H}_{2} \mathrm{O}\right)$ or IC (DFO) for 2 or $16 \mathrm{hrs}$ followed by Tfrc smRNA FISH analysis.

I, Violin plot showing total Tfrc mRNA molecules per cell. For $2 \mathrm{~h}, \mathrm{n}=71$ (Control) and $\mathrm{n}=74$ (IC); for $16 h, n=62$ (control) and $n=66$ (IC).

m, Violin plot showing Tfrc nascent mRNA molecules per transcription site (TS). For $2 \mathrm{~h}, \mathrm{n}=19$ (Control) and $n=31$ (IC); for 16h, $n=21$ (control) and $n=14$ (IC).

n, Histogram showing frequency distribution of active Tfrc transcription sites (TS) per cell in nonmitotic and mitotic cells.

Significance $p$-values, indicated as ${ }^{*} p<0.05$, ${ }^{* *} p<0.01$, ${ }^{* * *} p<0.001,{ }^{* * * *} p<0.0001$, were calculated using Student's t-test (unpaired: i, I and m) and two-sample Kolmogorov-Smirnov tests (h).

\section{Extended Data Figure 3. Iron chelator-mediated induction of the limited-iron response in HSPC.}

a, Ftl1 protein quantification in HPC-7 cells at 1 and 2hrs after IC (IC1: DFO, IC2: EP) exposure. Western blot images (middle) and quantification (right).

b, Immunofluorescence and confocal microscopy analysis of Ncoa4-dependent ferritinophagy in HSC after IC treatment for 48hrs. The number of Noca4 puncta in each single HSC was enumerated, and the frequency of cells with at least one Ncoa4 punctum is shown.

c, Quantification of alteration in intracellular $\mathrm{Fe}^{2+}$ content after IC (EP) treatment of HPC-7 cells using FeRhoNox-1 and FACS analysis. Scheme (left) and representative FACS plots (right) at different time points. $n=6 ; 2$ independent experiments.

d, Left: Scheme of microarray analysis to identify expression changes in HSC after 16hrs IC (DFO) exposure compared to mock treatment controls. Right: Volcano plot showing the 
distribution of expression changes. Significantly down- or up-regulated genes are highlighted, and IRE-containing genes are denoted with triangles, includes Tfrc and Slc40a1.

e, Gene expression changes in purified HSC 16hrs after IC exposure (IC1: DFO) compared to mock treatment controls by microarray identifies differential levels of IRE-containing transcripts (pie chart) and includes Tfrc and Slc40a1 (IRE structures shown as example).

f, Alterations in the mRNA expression of the iron regulatory pathway assessed by qRT-PCR (upper IC1: DFO; lower IC3: DFX), $\mathrm{n}=3-5$.

g, Cell surface CD71 presentation on HSC 4-6hrs upon iron chelator (DFO) exposure by FACS analysis, $\mathrm{n}=4$.

h, Quantification of the labile iron pool in HSPC populations. Scheme shows cells preloaded with Calcein-AM exposed to IC or vehicle treatment in growth medium. Data represents the increase in Calcein-AM mean fluorescence intensity (MFI) upon IC treatment (\% vehicle control). $n=4$.

If not specified differently, data are mean \pm SEM. (c, $\mathbf{f}, \mathbf{g}, \mathbf{h})$. Significance $p$-values, indicated as ${ }^{\star} p<0.05,{ }^{* *} p<0.01,{ }^{* *} p<0.001$, calculated by Student's $t$-test (paired: $\mathbf{f}, \mathbf{g}, \mathbf{h}$; unpaired: $\mathbf{c}$ ), or Hypergeometric test (e).

\section{Extended Data Figure 4. Activation of the limited-iron response increases HSPC.}

a, Distribution of union HSC signature expression in phenotypic HSC (green) and non-HSC (orange). Average expression of the signatures in each of the single cells were calculated. Density plot shows the Kernel density estimate of the distribution of HSC signature expression in phenotypic HSC versus non-HSC.

b,c, Comparison of the expression of IC-treated HSC versus control HSC in scRNA sequencing, with gene signatures associated with HSC quiescence (b, GSE108155) and stemness (c). Lists of the gene signatures are available in Extended Data Table 1. Signature scores were calculated with Seurat package.

d, Comparison of the expression of IC-treated HSC versus control HSC in ScRNA sequencing with the cycling signatures (GSE113495).

$\mathbf{e}, \mathbf{f}$, Quantification of protein levels of Ferritin light chain (e) and transferrin (f) in the serum of mice exposed to in vivo treatment of IC (DFO) or vehicle control for 2 weeks.

$\mathbf{g}$, Complete blood count (CBC) analysis on peripheral blood of mice after in vivo iron chelator (IC) or mock control (control) treatment. Quantification of red blood cells (RBC), hemoglobin, and mean corpuscular volume (MCV). $\mathrm{n}=4-6$.

h, Quantification of functional HSC after in vivo iron chelator (IC: DFO) treatment using LTC-IC assay. Left: bar plot shows the LTC-IC frequencies across individual mice in the group of IC or control. $n=5$ (control) and $n=9$ (IC). Estimated group LTC-IC frequencies by ELDA (right); ELDAcalculated LTC-IC frequencies for both groups in parentheses in ELDA plot (right).

i, Experimental strategy for pharmacological inhibition of iron import and intracellular mobilization.

j, k, LTC-IC assay to quantify HSC frequency in LSK cells without and with heterozygous deletion of Fth1 (i), and HSC frequency in Fth1-het LSK following mock or IC (DFO) treatment (j), $\mathrm{n}=4$. 
1485

1486

1487

1488

1489

1490

1491

1492

1493

1494

1495

1496

1497

1498

1499

1500

1501

1502

1503

1504

1505

1506

1507

1508

1509

1510

1511

1512

1513

1514

1515

1516

1517

1518

1519

1520

1521

1522

1523

1524

1525

1526

1527

If not specified differently, data are mean \pm SEM. (e, $\mathbf{f}, \mathbf{g} ; \mathbf{h}, \mathbf{k}$ (bar graph)). Significance $p$-values, indicated as ${ }^{*} p<0.05,{ }^{* *} p<0.01,{ }^{* * *} p<0.001,{ }^{* * * *} p<0.0001$, calculated by Student's $t$-test (paired: g; unpaired: b-f), or Poisson statistics: $\mathbf{h}, \mathbf{j}, \mathbf{k}$ (ELDA analysis).

\section{Extended Data Figure 5. Activation of the limited iron response stimulates fatty acid oxidation in HSPC.}

a. GSEAPreranked analyses of scRNA-seq data using MSigDB gene set HALLMARK_GLYCOLYSIS.

b,c, Following IC (DFO) treatment, HPC-7 cells were subjected to metabolite profiling (b). Differential metabolites were subjected to pathway analysis with IPA. c, Pathway network analysis of differential metabolites in HPC-7 cells after 12-hours IC exposure, $n=4$.

d-f, d, Scheme for assessing the transcriptional alterations triggered by iron chelation. Sorted HSC were subjected to ex vivo treatment with DFO for 16 hours, followed by microarray analysis. IPA pathway analysis was performed with differentially expressed genes (DEG; average fold change $>1.6$ and $p<0.05 ; n=4)$. e,f, Pathway network analysis of DEG by IPA canonical pathway analysis $(\mathbf{f})$, and biological functions $(\mathbf{f})$, and significantly enriched terms $(p<0.05)$ were subjected to EnrichmentMap for network visualization, and clustered with AutoAnnotate.

g-i, Metabolite profiling of mouse Lin-Sca- $1^{+} \mathrm{CKit}^{+}$(LSK) (h) and HPC-7 cells (i) following the ex vivo treatment with iron chelators for $12 \mathrm{hrs}(\mathbf{g})$. $\mathbf{h}$,i, IPA biological function analysis performed with metabolites showing significant changes upon IC (DFO) treatment (with an average fold change > 1.3) compared to mock treatment controls. Pathways related to fatty acid and lipid metabolism are shown for LSK $(\mathbf{h}, n=2)$, HPC-7 cells $(\mathbf{i}, n=4)$.

j, VPS34i reverses differential abundance upon IC (DFO) exposure of almost half of the metabolites, including a significant number of metabolites belonging to fatty acid and lipid metabolic pathways as indicated. Restored metabolites were defined as those with average fold change > 1.25 (IC1 versus IC1+VPS34i; $n=3)$.

k-m, k, Hadha staining in HPC-7 cells exposed to iron chelators (IC1-lo: DFO $5 \mu \mathrm{M}$, IC1-hi: DFO $10 \mu \mathrm{M}$, or IC2-lo: EP $3 \mu \mathrm{g} / \mathrm{ml}$, IC2-hi: EP $10 \mu \mathrm{g} / \mathrm{ml}$ ) or respective mock treatment controls for $24 \mathrm{hrs}$. I,m, Quantification of Hadha MFI upon the treatment of IC1 (I) or IC2 (m), representative histograms and fold changes of Hadha mean fluorescence intensity (MFI) values compared to mock controls are shown. $\mathrm{n}=3$.

$\mathbf{n}, \mathbf{0}, \mathbf{n}$, Assessment of fatty acid metabolism in hematopoietic progenitor cell line, $\mathrm{Ba} / \mathrm{F} 3$, by Seahorse analysis detecting total OXPHOS (control) and $\beta$-oxidation (after CPT-1 inhibition with etomoxir (Etx)) specific oxygen consumption rates (OCR) in the presence or absence of IC (DFO), without (left) or with (right) inhibition on lipolysis using diethylumbelliferyl phosphate (DEUP). $\mathbf{0}$, representative analysis plots.

p, Total (left) and FAO-specific (right) mitochondrial ATP production measured by Seahorse analysis. $\mathrm{n}=3$.

q, LTC-IC assay to quantify functional HSC upon or IC2 (EP) treatment alone or in combination with pharmacological inhibition of fatty acid import to mitochondria using etomoxir (Etx). Fold changes of long-term culture-initiating cell (LTC-IC) frequencies compared to controls across individual mice (bar graph). In the ELDA plot, average stem cell frequency is shown for each treatment group. $\mathrm{n}=7$. 
If not specified differently, data are mean \pm SEM. (p, q). Significance $p$-values, indicated as * $p<$ $0.05,{ }^{* *} p<0.01,{ }^{* \star *} p<0.001,{ }^{* * \star} p<0.0001$, were calculated by Student's $t$-test (paired: I, $\mathbf{m}$ ).

Extended Data Figure 6. $\beta$-oxidation of arachidonic acid increases HSPC pool upon limited 1532 iron response activation.

a, Protein expression of Ptgs1 in HPC-7 cells upon treatment with IC1 (DFO), IC2 (EP) or in mock treatment controls at 8, 16 and 24hrs after IC exposure. Representative images (left panels) and fold changes of actin-normalized protein levels by image analysis compared to vehicle control are shown (right graphs).

1536

b, Protein expression of genes involved in arachidonic acid metabolism in HSC versus progenitors $\mathrm{n}=3$.

c,d, Genetic inhibition of Acyl-CoA Synthetase Long Chain Family Member 4 (Acs/4) by RNAi in primary cKit+ bone marrow mononuclear cells using two independent shRNA targeting Acs/4 (Acsl4 sh\#3, Acs/4 sh\#4) or a non-targeting control (NTC). Quantification of Acs/4 mRNA expression (c) and protein levels (d) in successfully transduced (GFP+) cells 48hrs after lentiviral transduction, $\mathrm{n}=3$.

1543

1544

e, Assessment of fatty acid oxidation rates in BA/F3 cells transduced with non-targeting control (NTC) or shRNA targeting Acs/4 (KD) after IC (DFO) treatment. Experimental scheme and representative OCR plots for NTC (left plots)) and Acs/4 knockdown (right plots) cells.

1548

f, Assessment of fatty acid oxidation rates in BA/F3 cells transduced with non-targeting control

1550 (NTC) or shRNA targeting Acs/4 (KD) under steady state. Basal and maximal fatty acid-specific OCRs are shown, $\mathrm{n}=3$.

If not specified differently, data are mean $\pm \operatorname{SEM}(\mathbf{b}, \mathbf{c}, \mathbf{f}) .{ }^{*} \mathrm{p}<0.05,{ }^{* *} \mathrm{p}<0.01,{ }^{* * *} \mathrm{p}<0.001$, n.s. not significant, calculated by Student's $t$-test (unpaired: $\mathbf{b}, \mathbf{c}$; paired: $\mathbf{f}$ )

1553

\section{Extended Data Figure 7. Increased HSC self-renewal associated gene expression upon iron} limitation response activation.

a, Quantification of HSC by LTC-IC assay after the single treatment with inhibitor of ATP citrate 1557 lyase (ACLY).

b, GSEAPreranked analyses of scRNA-seq data using MSigDB gene set KAT5_TARGET_GENES (human cells), and common binding targets of Tip60 and Myc. Common binding targets of Tip60 and Myc were obtained by comparing the ChIP-seq of Tip60 in ESC and Myc in $\mathrm{CH} 12$ and MEL cells published previously (GSE49847). Only genes bound by Myc in both $\mathrm{CH} 12$ and MEL cells were considered as Myc target in this analysis.

c, Overlap of DEG in IC-treated HSC by scRNA sequencing with those in Tip60-KO LSK cells. Number of unique and shared DEG are shown. Significance of overlap was calculated using Hypergeometric tests. 
1569

1570

1571

1572

1573

1574

1575

1576

1577

1578

1579

1580

1581

1582

1583

1584

1585

1586

1587

1588

1589

1590

1591

1592

1593

1594

1595

1596

1597

1598

1599

1600

1601

1602

1603

1604

1605

1606

1607

1608

1609

1610

1611

vehicle controls (grey). f, GSEAPreranked analysis using MSigDB collection gene sets of validated targets upon c-Myc transcriptional activation (PID_MYC_ACTIV_PATHWAY) and repression (PID_MYC_REPRESS_PATHWAY), as well as HALLMARK_MYC_TARGETS_V1.g, Upper panel: overlap of differentially expressed genes (DEG) in IC-treated HSC (vs. vehicle control) and HSC lacking $c-M y c$ and $n-M y c$ (Myc-dKO) ${ }^{147}$. Number of unique and shared DEG are shown. Significance of overlap was calculated using Hypergeometric tests. Lower panel: GSEAPreranked analyses using DEG in Myc-dKO versus wildtype. $\mathbf{h}$, GSEAPreranked analyses with gene set of DEG upon JQ1-treatemt in K562 and MV4-11 cells ${ }^{148}$.

i, IPA upstream regulator analysis comparing DEG in IC-treated HSC, Myc-dKO HSC ${ }^{147}$, and Tip60-KO LSK ${ }^{72}$. Z-scores of top 15 activated and inhibited (ranked by Z-score) upstream regulators and corresponding Z-scores in Myc-dKO HSC and Tip60-KO LSK are shown.

j, IPA upstream regulator analyses of DEG (absolute fold change > 1.5, p-value $<0.05$ ) in Fbx/5deficient HSC (Fbxl5-KO) ${ }^{43}$ were compared to gene expression alterations seen in Myc-dKO HSC $^{147}$ and Tip60-KO LSK (vs. wildtype controls). Z-scores of top 15 activated (ranked by Zscore) and inhibited upstream regulators in Fbx/5-KO HSC and corresponding Z-scores in MycdKO HSC and Tip60-KO LSK are shown.

k, GSEA analysis of expression profile of FbxI5-KO HSC was performed with DEG in Myc-dKO HSC versus wildtype controls; significantly up-regulated DEG enrichment analysis (orange) and down-regulated genes (light orange).

I, Expression changes of target genes in HSC after IC treatment alone or in combination with CDK8/CDK19 inhibitors (CA: Cortistatin A; SA: Senexin A) by Fluidigm analysis. Fold changes of genes across treatment groups from Fluidigm and scRNA-seq (SC) are shown. Significance of differential expression shown (right panel) were estimated by paired Student's $t$-test comparing the $\Delta^{\mathrm{Ct}}$ in IC versus control, co-treatment versus IC. ${ }^{*} \mathrm{p}<0.05,{ }^{\star *} \mathrm{p}<0.01,{ }^{* \star *} \mathrm{p}<0.001, n$, not significant.

m-o, Quantification of HSC by LTC-IC assay after the single treatment with inhibitors of Tip60 or Myc $(\mathbf{m})$, CDK8/CDK19 (SA: Senexin A, o) or in combination with IC (n). Average LTC-IC frequencies are shown in parentheses. Significance tested using ELDA. Significance $p$-values, indicated as ${ }^{*} p<0.05$, n.s. not significant, calculated by Poisson statistics.

\section{Extended Data Figure 8. Cytoplasmic iron loading compromises function of aged HPSC.}

a,b, Increased labile iron pool (LIP) in aged hematopoietic stem and progenitors compared to young mice. Cytoplasmic $\mathrm{Fe}^{2+}(\mathbf{a})$ or $\mathrm{Fe}^{3+}(\mathbf{b})$ LIP were measured using FeRhoNox-1 and CalceinAM staining, respectively. Quantification of FeRhoNox-1 and Calcein-AM mean fluorescence intensity (MFI in arbitrary units (a.u.)) by FACS analysis are shown. $n=4-9$.

c,d, Quantification of protein levels of Ferritin light chain (c) and transferrin (d) in the serum of aged versus young mice.

e, IRE enrichment analysis with DEG of murine aged HSC compared to young HSC in GSE47817. Significance of enrichment was calculated by hypergeometric test.

$\mathbf{f}-\mathbf{h}$, Comparison of the expression of Ftl1 (f), Fth1 $(\mathbf{g})$, and Tfrc (h) in HSC isolated from young and aged mice. Single cell RNA sequencing data of aged and young HSC was obtained from previous study (GSE59114); HSC with Von Willebrand Factor ( Vwf) expression >3 were defined as $\mathrm{Vwf}+$, The expressions of Ftl1, Fth1, and Tfrc were compared across different groups. 
1612 i, Pathway analysis of genes in the leading edge of GSEA shown in Fig. $\mathbf{4 g}$.

1613 j, IPA upstream regulator analysis comparing DEG in Myc-dKO HSC ${ }^{147}$, Tip60-KO LSK ${ }^{72}$, as well 1614 as DEG in aged versus young Vwf+ HSC from previous studies (GSE59114 and GSE70657). Z1615 scores of top 15 activated and inhibited (ranked by Z-score) upstream regulators and their 1616 corresponding Z-scores are shown.

1617 k-n, Quantification of megakaryocyte colony-forming units in aged HSC compared to young HSC

1618

1619

1620

1621

1622

1623

1624

1625

1626

1627

1628

1629

1630

1631

1632

1633

1634

1635

1636

1637

1638

1639

1640

1641 at baseline level (k). CFU-Mk derived from aged HSC in response to Tip60 or Myc inhibitors alone (I), or in combination with iron chelators $(\mathbf{m}, \mathbf{n})$. Quantification of CFU-Mk colonies across different conditions was normalized to 2000 HSC plated. $n=3-8$.

If not specified differently, significance $p$-values, indicated as ${ }^{*} p<0.05$, ${ }^{* *} p<0.01,{ }^{* * *} p<0.001$, ${ }^{* * * *} p<0.0001$, were calculated using Student's t-test (unpaired: $\mathbf{a}-\mathbf{d}, \mathbf{f}-\mathbf{h}, \mathbf{j}$, and paired: $\mathbf{k}-\mathbf{m}$ ).

\section{Extended Data Figure 9. Proposed cell functional and molecular model.}

Extended Data Table 1. List of gene signatures.

Extended Data Table 2. Differentially expressed genes in DFO-treated HSC by Microarray analysis.

Extended Data Table 3. Differentially expressed genes in DFO-treated HSC by scRNA-seq.

Extended Data Table 4. Pathway enrichment of differentially expressed genes in DFO-treated HSC by Microarray analysis.

Extended Data Table 5. Pathway enrichment of differentially expressed genes in DFO-treated HSC by scRNA-seq.

Extended Data Table 6. Altered metabolites in HPC7 cells with the treatment of DFO.

Extended Data Table 7. Altered metabolites in mouse LSK cells with the treatment of DFO.

Extended Data Table 8. Biological function enrichment (IPA analysis) of altered metabolites in DFO-treated HPC7 cells.

Extended Data Table 9. Biological function enrichment (IPA analysis) of altered metabolites in DFO-treated LSK cells.

Extended Data Table 10. List of antibodies used in the study.

Extended Data Table 11. List of oligos used in this study.

Extended Data Table 12. List of published data sets used for integrative analysis. 